\title{
Conocimiento taxonómico de la familia Poaceae en México
}

\author{
Patricia Dávila ${ }^{1 *}$, Ma. Teresa Mejia-Saulés², Ana María Soriano- \\ Martínez $^{3}$ y Yolanda HerRera-Arrieta ${ }^{4}$
}

Botanical Sciences

96 (3): 462-514, 2018

DOI: $10.17129 /$ botsci.1894

Received:

October 18th, 2017

Accepted:

April 21st, 2018

Editor asociado:

Guillermo Ibarra-Manriquez

\section{Resumen}

Antecedentes La clasificación de la familia Poaceae ha cambiado a lo largo de los años, por la evolución de distintas áreas del conocimiento de la sistemática, tanto en sus aspectos teóricos como prácticos. La morfología y anatomía siguen siendo fuentes importantes de información taxonómica en las gramíneas. Sin embargo, en los últimos 20 años, los trabajos moleculares que buscan inferir la filogenia de los diferentes taxa, han dominado el escenario de la sistemática de la familia, lo que ha derivado en cambios y re-arreglos a todos los niveles taxonómicos.

Objetivo: Realizar la revisión y actualización de la información taxonómica y geográfica de las Poaceae de México, presentando un listado de especies, en el que se señalan los taxa nativos, introducidos y endémicos, los logros alcanzados en el conocimiento del grupo, así como los retos que se habrán de enfrentar en el futuro para adelantar en el conocimiento de las gramíneas de México.

Métodos: Se tomó como base de referencia el trabajo de Dávila et al. (2006). Se revisó tanto la literatura especializada disponible de 2006 a la fecha y distintas bases de datos nomenclaturales y taxonómicas. El trabajo también incluye la revisión de ejemplares de 29 herbarios entre 2006-2017. La lista de las especies se presenta con base en la clasificación de Soreng et al. (2015).

Resultados: Se registran en México 11 subfamilias, 205 géneros, 1,216 especies y 207 categorías infraespecíficas. Se presenta el listado alfabético de las especies, resaltando su distribución a nivel estatal y en particular las endémicas e introducidas. Se incluye también la distribución de las especies a nivel estatal. Asimismo, se presentan los avances alcanzados en relación a los estudios de las diferentes subfamilias con diversos enfoques de estudio, tanto metodológicos como conceptuales, y los retos que se habrán de enfrentar en los próximos años.

Palabras clave: clasificación, gramíneas, nomenclatura, pastos.

\section{Abstract}

Background: The classification of the Poaceae family has changed over the years, due to the knowledge evolution, the use of different techniques and theoretical approaches and the technological advances. Important sources of taxonomic information on grasses are still their morphology and anatomy. However, in the past 20 years, molecular work seeking to infer the phylogeny of different taxa has dominated the Systematics of the Poaceae. This knowledge trend has resulted in changes and rearrangements at all taxonomic levels.

Objective: To carry out the review and comprehensive updating of the taxonomic and geographic information of the Poaceae of Mexico, including the species checklist, highlighting the native, introduced and endemic taxa, as well as, the achievements obtained and the future research challenges.

Methods: The main reference used was the work of Dávila et al. (2006). We also undertook a thorough revision of the available specialized literature since 2006 and the nomenclatural and taxonomic databases. This work also included the specimens' revision from 29 different herbaria that were consulted between 2006-2017. The species checklist follows the classification of Soreng et al. (2015).

Results: The grasses recorded in the country belong to 11 subfamilies, 205 genera, 1,216 species and 207 infraspecific categories. A species alphabetical list is included, highlighting the introduced and endemic taxa. In addition, the species distribution, at the state level, is also presented. Also data regarding the achievements obtained and the challenges for the coming years, is included.

Key words: classification, grasses, nomenclature, pastures.
This is an open access article distributed under the terms of the Creative Commons Attribution License CC BY-NC (4.0) international. 


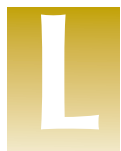

a clasificación de las Poaceae ha cambiado a lo largo del tiempo debido a las importantes aportaciones realizadas por agrostólogos en distintas partes del mundo, quienes han propuesto cambios en la delimitación taxonómica a distintos niveles jerárquicos. Estas aportaciones incluyen, para cada subfamilia, lo siguiente: subfamilia Anomochlooideae: Morris \& Duvall 2010 (Anomochloa marantoidea); subfamilia Bambusoideae: Bamboo Phylogeny Group 2012, Kelchner \& Bamboo Phylogeny Group 2013; subfamilia Chloridoideae:Peterson et al. 2010b (Muhlenbergiinae), 2010a (Chloridoideae), 2011 (Centropodieae), 2014a, 2015b (Cynodonteae), Snow et al. 2013 (Disakisperma); subfamilia Danthonioideae: Baker et al. 2007, Pirie et al. 2009, Linder et al. 2010; subfamilia Panicoideae: Sede et al. 2008, 2009 (Paniceae), Chemisquy et al. 2010 (Pennisetum, Cenchrus y Odontelytrum), Zuloaga et al. 2010 (Panicum); subfamilia Pooideae: Cialdella et al. 2007, 2014, Romaschenko et al. 2008, Sclovich et al. 2015 (Stipeae), Döring et al. 2007 (complejo Aveneae/Poeae), Refulio-Rodriguez et al. 2012 (Dissanthelium). Los resultados de estos estudios han sido la base para proponer una clasificación filogenéticamolecular mundial de las Poaceae (Soreng et al. 2015).

El trabajo taxonómico, como cualquier otra disciplina científica, fue estimulado en las últimas décadas por los avances tecnológicos que permitieron tener disponibles equipos y programas sofisticados que facilitaron el desarrollo de estudios cada vez más especializados, así como la construcción y manejo de bases de datos y el análisis de la información utilizando diferentes algoritmos. Sin lugar a dudas, las hipótesis de clasificación actuales están casi siempre basadas en los trabajos filogenéticos que han utilizado, entre otras técnicas, la biología molecular, combinándola con información morfológica, anatómica y cromosómica. Entre las hipótesis de clasificación destacan: Linder 2010 (clasificación genérica de Danthonioideae), Peterson et al. 2010b (clasificación de la subfamilia Chloridoideae), 2012, 2014a, 2015a, (clasificación de Cynodonteae), 2014b (clasificación de Sporobolinae), Snow et al. 2013 (sistemática de Disakisperma), Sánchez-Ken \& Clark 2010 (clasificación de Panicoideae), Bamboo Phylogeny Group 2012, Kelchner \& Bamboo Phylogeny Group 2013 (clasificación de Bambusoideae), y Soreng et al. 2015 (clasificación de la familia Poaceae).

Por lo tanto, el presente estudio tiene como objetivo principal presentar una lista taxonómica actualizada de las gramíneas de México, con base en la propuesta de clasificación de Soreng et al. (2015), señalando los taxa nativos, introducidos y endémicos y su distribución a nivel estatal. Adicionalmente, se describen los avances alcanzados en el conocimiento de este grupo vegetal y los retos que se habrán de enfrentar en los próximos años para avanzar en el conocimiento de las gramíneas de México.

\section{Materiales y métodos}

revisó el borrador.

Ana María Soriano Martínez se encargó de la revisión taxonómica de las subfamilias Anomochlooideae, Aristoideae, Arundinoideae, Danthonioideae, Micrairoideae, Oryzoideae, Panicoideae, Pharoideae, contribuyó en la elaboración de la lista de especies, apoyó en la redacción del artículo y revisó el borrador. Yolanda Herrera Arrieta se encargó de la revisión taxonómica de la subfamilia Chloridoideae, contribuyó en la elaboración de la lista de especies, apoyó en la redacción del artículo y revisó el borrador.

El Catálogo de Gramíneas de México, elaborado por Dávila et al. (2006), fue la base para actualizar las Poaceae de México, en tanto que la clasificación filogenética utilizada correspondió a la propuesta por Soreng et al. (2015). Para dicha actualización se revisó la bibliografía publicada desde 2006 a la fecha, incluyendo artículos científicos, monografías, revisiones taxonómicas, tesis, floras e inventarios florísticos (Tablas 5 y 6). Se consultaron la base de datos bibliográficos del INECOL (ACSESS, IEEE Xplore, JSTOR, WoS, Scopus, Knovel, Royal Society-Journals), eBooks (EBSCO, CABI ebooks, Elsevier-Journals-eBooks, ProQuest (Dissertation \& Theses), y Springer-Journals-eBooks.

Además, se consultaron tres bases de datos botánicos: Tropicos (Missouri Botanical Garden, 2016), "REMIB" (CONABIO, 2016) y "The Plant List" (Royal Botanic Gardens, Kew, Missouri Botanical Garden, 2016).

Asimismo, se revisaron los ejemplares botánicos de 29 herbarios en México y en el extranjero. A continuación se enuncian los acrónimos de dichos herbarios, de acuerdo a Thiers (con actualizaciones continuas): AMEX, ANSM, CAS, CFNL, CHAPA, CICY, CIIDIR, COCA, ENCB, FCME, BUG, IEB, IZTA, ENBC; KEW, MEXU, MICH, MO, NY, RSA, SLPM, TAES, TEX, UADY, UAT, UC, UJAT, US, XAL. Estas colecciones fueron revisadas total o parcialmente por algunas de las autoras en el periodo comprendido entre 2006 y 2017. La experiencia taxonómica de las autoras fue considerada para realizar la actualización, obtener resultados confiables y contar con la bibliografía actualizada. Se elaboró el mapa de distribución 
de las gramíneas de México (Figura 1), a partir de la base de datos obtenida de la Red Mundial de Información sobre Biodiversidad (REMIB) de la Comisión Nacional para el Conocimiento y Uso de la Biodiversidad (CONABIO 2017) con base en 32,455 registros.

\section{Resultados}

Diversidad. Los resultados de este trabajo indican que en la República Mexicana se registra la presencia de 11 subfamilias, 205 géneros, 1,216 especies (1,062 nativas y 154 introducidas), y 207 categorías infraespecíficas de gramíneas (Apéndice 1). Las subfamilias con el mayor número de especies son (Tabla 1): Panicoideae (482 especies), Chloridoideae (348) y Pooideae (260).

Al revisar la diversidad de las Poaceae mexicanas, se destaca que 10 géneros (Muhlenbergia, Paspalum, Bouteloua, Panicum, Eragrostis, Sporobolus, Aristida, Digitaria, Festuca y Setaria) (Tabla 2), aportan un poco más de $42 \%$ del total de especies existentes en el país (514). Además, tres de ellos (Muhlenbergia, Bouteloua y Festuca), también tienen un alto porcentaje de especies endémicas de México y carecen de taxa introducidos o cultivados (Tabla 2). En contraste, 87 géneros ( $43 \%$ ) tienen una sola especie en México, aunque no todos son monotípicos.

A nivel estatal, las entidades federativas con mayor riqueza agrostológica nativa son Chiapas (508 taxa), Veracruz (490), Oaxaca (468), Jalisco (423), Puebla (384) y Estado de México (381) (Tabla 3). Las entidades federativas, con mayor número de especies, en cada subfamilia, se presentan en la Tabla 4. Destacan por su diversidad de especies, las subfamilias Chloridoideae especialmente en las zonas cálidas y secas, Panicoideae en regiones cálidas y húmedas y Pooideae en zonas templadas.

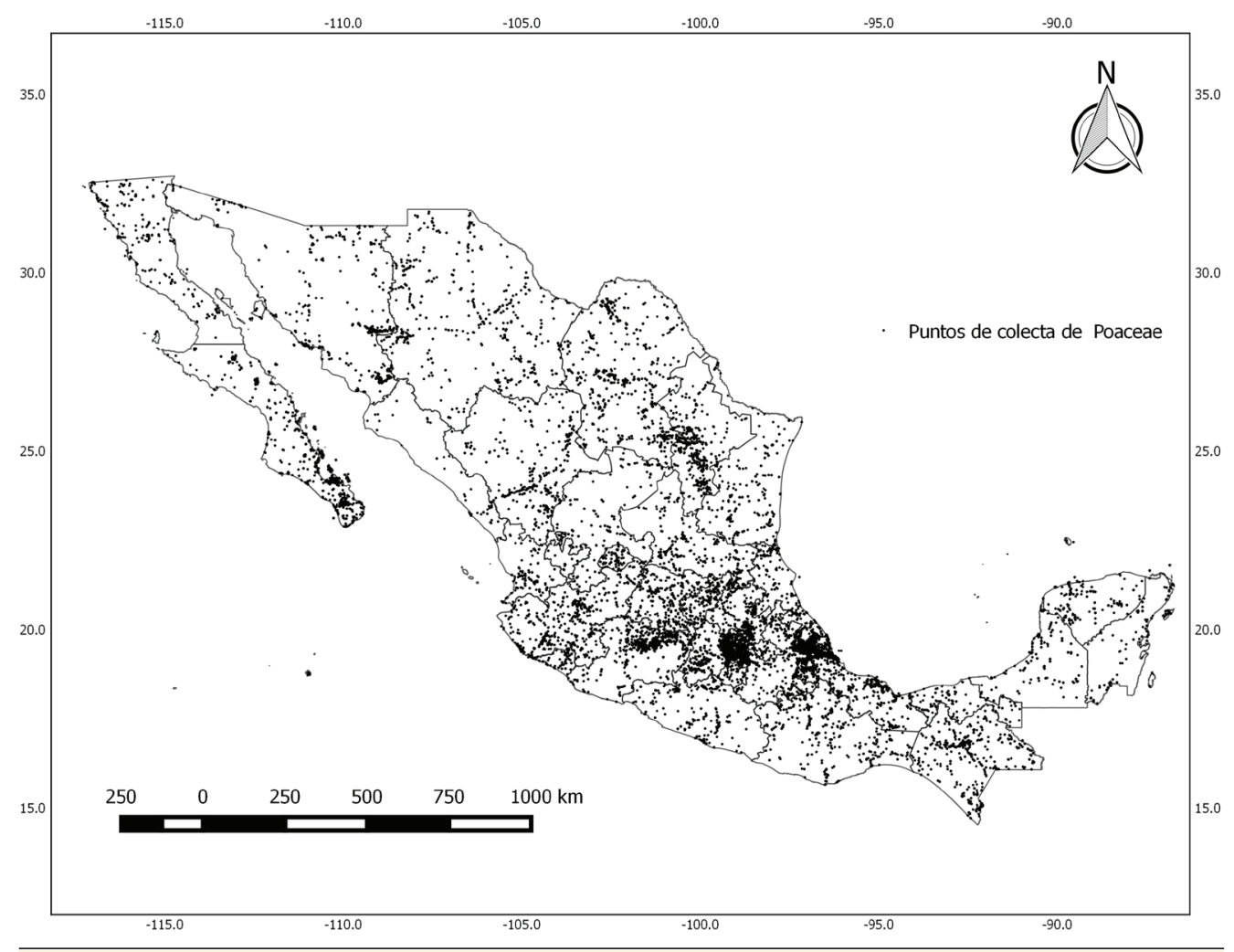

Figura 1. Sitios de colecta de diferentes grupos de la familia Poaceae. La información para la elaboración de este mapa se obtuvo de la base de datos de la Red Mundial de Información sobre Biodiversidad (REMIB), de la Comisión Nacional para el Conocimiento y Uso de la Biodiversidad (CONABIO, 2017). 
Tabla 1. Diversidad de las subfamilias de Poaceae en México. Para cada subfamilia se indica el total de especies, las endémicas y las introducidas o cultivadas. En cada caso, se incluye también la información de sus respectivas categorías infraespecíficas (variedad/forma/subespecie).

\begin{tabular}{lccc} 
Subfamilia & Total de especies & Taxa endémicos & Taxa introducidos o cultivados \\
\hline Anomochlooideae & $2(0 / 0 / 0)$ & & \\
Aristidoideae & $33(17 / 6 / 0)$ & $13(2 / 2 / 0)$ & $1(0 / 2 / 0)$ \\
Arundinoideae & $2(0 / 2 / 0)$ & & $8(0 / 0 / 0)$ \\
Bambusoideae & $63(0 / 0 / 2)$ & $32(0 / 0 / 2)$ & $35(2 / 0 / 0)$ \\
Chloridoideae & $348(38 / 1 / 8)$ & $111(3 / 0 / 1)$ & $3(0 / 0 / 0)$ \\
Danthonioideae & $6(0 / 0 / 1)$ & $1(0 / 0 / 0)$ & $2(0 / 0 / 0)$ \\
Micrairoideae & $3(0 / 0 / 0)$ & & $53(5 / 2 / 0)$ \\
Oryzoideae & $13(3 / 0 / 0)$ & $1(1 / 0 / 0)$ & $52(4 / 0 / 3)$ \\
Panicoideae & $482(73 / 0 / 7)$ & $66(1 / 0 / 1)$ & $154(11 / 4 / 3)$ \\
Pharoideae & $4(0 / 0 / 0)$ & & \\
Pooideae & $260(25 / 0 / 24)$ & $71(6 / 0 / 6)$ & \\
\hline TOTAL & $1216(156 / 9 / 42)$ & $295(13 / 2 / 10)$ & \\
\hline
\end{tabular}

Endemismo. En México, se registran 315 taxa (295 especies) endémicos a su territorio (Tabla 1). Destaca el hecho que $32.6 \%$ de las especies (95) son conocidas de un solo estado y que $15 \%$ (44), se registran solamente en dos estados. En contraste, casi $9 \%$ de las especies endémicas (26) se distribuyen en 10 o más estados de la República Mexicana.

Un total de cinco géneros (Muhlenbergia, Paspalum, Bouteloua, Panicum y Eragrostis) aportan $35.3 \%$ del total de las especies de gramíneas endémicas de México (Tabla 2). En contraste, cuatro géneros (Aulonemia, Merostachys, Metcalfia y Sohnsia) tienen una sola especie en México y ésta es endémica al país. Sin embargo, a nivel mundial solamente Sohnsia es un género monotípico, mientras que Aulonemia tiene 50 especies, Merostachys 47 y Metcalfia 4.

A nivel estatal (Tabla 3), las entidades que sobresalen por el número más elevado de taxa endémicos a México son Jalisco (84), Puebla (77), Durango (68), Michoacán (66) y Veracruz (58).

Los géneros con mayor número de especies endémicas de México, organizados por subfamilia, son los siguientes (Apéndice 1): Aristidoideae, con Aristida (13 especies endémicas); Bambusoideae, con Chusquea (12), Otatea (9) y Olmeca (4); Chloridoideae, con Muhlenbergia (50), Bouteloua (25), Sporobolus (9); Danthonioideae, con Danthonia (1); Panicoideae, con

Tabla 2. Géneros de gramíneas más diversos de México. Para cada género se indica el total de especies que presentan, así como la cantidad y porcentaje de especies endémicas e introducidas o cultivadas que tienen.

\begin{tabular}{lccc} 
Géneros & Número de especies & Endémicas $\mathbf{( \% )}$ & Introducidas o cultivadas $\mathbf{( \% )}$ \\
\hline Muhlenbergia & 127 & $50(40)$ & $0(0)$ \\
Paspalum & 87 & $14(17)$ & $1(1)$ \\
Bouteloua & 52 & $25(42)$ & $0(0)$ \\
Panicum & 47 & $6(12)$ & $3(6)$ \\
Eragrostis & 45 & $5(6)$ & $12(26)$ \\
Sporobolus & 34 & $9(23)$ & $2(6)$ \\
Aristida & 33 & $13(39)$ & $0(0)$ \\
Digitaria & 31 & $5(16)$ & $6(19)$ \\
Festuca & 30 & $18(60)$ & $0(0)$ \\
Setaria & 28 & $2(7)$ & $2(7)$ \\
\hline
\end{tabular}


Tabla 3. Riqueza florística por entidades federativas. En la segunda columna se incluye el total de taxa registrados para la entidad y entre paréntesis, el porcentaje del total conocido para México. En la tercera columna se indica el número total de taxa endémicos de México que se encuentran en la entidad y entre paréntesis, la cantidad de taxa endémicos exclusivos de la entidad. En la cuarta columna se presenta el total de taxa introducidos o cultivados y entre paréntesis, su porcentaje con respecto al total conocido para México.

\begin{tabular}{|c|c|c|c|c|}
\hline Entidad & $\begin{array}{c}\text { Taxa nativos } \\
(\%)\end{array}$ & $\begin{array}{c}\text { Taxa } \\
\text { endémicos }\end{array}$ & $\begin{array}{c}\text { Taxa cultivados o introducidos } \\
(\%)\end{array}$ & Total taxa registrados \\
\hline Chiapas & $508(42)$ & $45(9)$ & $65(11)$ & 573 \\
\hline Veracruz & $490(40)$ & $58(10)$ & $67(12)$ & 557 \\
\hline Oaxaca & $468(39)$ & $57(4)$ & $65(12)$ & 533 \\
\hline Jalisco & $423(35)$ & $84(9)$ & $55(12)$ & 478 \\
\hline Puebla & $384(32)$ & $77(2)$ & $56(13)$ & 440 \\
\hline Estado de México & $381(31)$ & $50(9)$ & $59(13)$ & 440 \\
\hline Chihuahua & $351(29)$ & $56(6)$ & $58(14)$ & 409 \\
\hline Durango & $348(29)$ & $68(4)$ & $44(11)$ & 392 \\
\hline Michoacán & $339(28)$ & $66(5)$ & $48(12)$ & 387 \\
\hline San Luís Potosí & $325(27)$ & $37(2)$ & $44(12)$ & 369 \\
\hline Coahuila & $305(25)$ & $37(8)$ & $50(14)$ & 355 \\
\hline Tamaulipas & $301(24)$ & $29(1)$ & $51(15)$ & 352 \\
\hline Nuevo León & $279(23)$ & $36(4)$ & $54(16)$ & 333 \\
\hline Sonora & $260(21)$ & $26(3)$ & $40(13)$ & 300 \\
\hline Zacatecas & $250(21)$ & 46 & $52(17)$ & 302 \\
\hline Guerrero & $246(20)$ & 38 & $30(11)$ & 276 \\
\hline Guanajuato & $240(20)$ & 41 & $34(12)$ & 274 \\
\hline Nayarit & 235 (19) & $38(5)$ & $30(11)$ & 265 \\
\hline Hidalgo & $228(19)$ & $30(2)$ & $39(15)$ & 267 \\
\hline Morelos & $223(18)$ & 24 & $41(16)$ & 264 \\
\hline Distrito Federal & $210(17)$ & 33 & $41(16)$ & 251 \\
\hline Baja California & $202(17)$ & $15(4)$ & $55(21)$ & 257 \\
\hline Querétaro & $200(16)$ & $25(1)$ & $27(12)$ & 227 \\
\hline Tabasco & $180(15)$ & 5 & $33(16)$ & 213 \\
\hline Sinaloa & $172(14)$ & 27 & $22(11)$ & 194 \\
\hline Aguascalientes & $160(13)$ & $23(1)$ & $30(16)$ & 190 \\
\hline Campeche & $159(13)$ & 6 & $37(19)$ & 196 \\
\hline Colima & $156(13)$ & $25(5)$ & $26(14)$ & 182 \\
\hline Yucatán & $153(13)$ & 7 & $42(22)$ & 195 \\
\hline Baja California Sur & $142(12)$ & $16(5)$ & $24(14)$ & 166 \\
\hline Quintana Roo & $122(10)$ & 4 & $27(18)$ & 149 \\
\hline Tlaxcala & $95(8)$ & 9 & $14(13)$ & 109 \\
\hline
\end{tabular}

Paspalum (14), Panicum (6), Zeugites (6), Urochloa (5); Pooideae, con Festuca (18), Trisetum (9), Agrostis (6), Poa, Achnantherum y Calamagrostis (5). En contraste, no se presentan especies endémicas en cuatro subfamilias: Anomochlooideae, Arundinoideae, Micrairoideae, Pharoideae.

Especies cultivadas o introducidas. Un total de 154 especies introducidas se registraron en el territorio mexicano. En particular, los seis géneros que tienen un mayor número de especies cultivadas o introducidas son: Eragrostis, Digitaria, Bromus, Cenchrus, Dactyloctenium 
Tabla 4. Entidades federativas, con mayor número de especies, en cada subfamilia.

\begin{tabular}{|c|c|c|}
\hline Subfamilias & Estados & Número de especies \\
\hline Anomochlooideae & Chiapas & 2 \\
\hline \multirow[t]{3}{*}{ Aristidoideae } & San Luis Potosí y Zacatecas & 27 \\
\hline & Coahuila & 26 \\
\hline & Chihuahua, Durango, Guanajuato y Puebla & 25 \\
\hline Arundinoideae & $\begin{array}{l}\text { Chihuahua, Coahuila, Colima, Guanajuato, Hidalgo, Jalisco, } \\
\text { México, Morelia, Nayarit, Nuevo León, Oaxaca, Puebla, } \\
\text { Querétaro, Sinaloa, Sonora, Tamaulipas y Veracruz }\end{array}$ & 2 \\
\hline \multirow[t]{3}{*}{ Bambusoideae } & Chiapas & 37 \\
\hline & Veracruz & 29 \\
\hline & Oaxaca & 21 \\
\hline \multirow[t]{3}{*}{ Chloridoideae } & Chihuahua & 183 \\
\hline & Durango & 165 \\
\hline & Jalisco & 154 \\
\hline Danthonioideae & Baja California, Chiapas, Chihuahua, Puebla, y Sonora & 3 \\
\hline Micrairoideae & Chiapas & 3 \\
\hline Oryzoideae & $\begin{array}{l}\text { Veracruz } \\
\text { Chiapas } \\
\text { Tabasco }\end{array}$ & $\begin{array}{c}11 \\
10 \\
7\end{array}$ \\
\hline Panicoideae & $\begin{array}{l}\text { Chiapas } \\
\text { Veracruz } \\
\text { Oaxaca }\end{array}$ & $\begin{array}{l}307 \\
267 \\
265\end{array}$ \\
\hline Pharoideae & $\begin{array}{l}\text { Chiapas y Veracruz } \\
\text { Tabasco } \\
\text { San Luis Potosí }\end{array}$ & $\begin{array}{l}4 \\
3 \\
2\end{array}$ \\
\hline Pooideae & $\begin{array}{l}\text { Michoacán } \\
\text { México } \\
\text { Puebla }\end{array}$ & $\begin{array}{c}109 \\
104 \\
87\end{array}$ \\
\hline
\end{tabular}

y Urochloa. En estos seis géneros se acumulan 36 taxa, lo cual equivale a $26 \%$ del total registrado.

En relación a estas especies, en un arreglo por subfamilias, sobresalen por su mayor cantidad en las entidades federativas, las siguientes (Apéndice 1): Arundinoideae - Chihuahua, Coahuila, Colima, Durango, Guanajuato, Guerrero, Hidalgo, Jalisco, México, Morelos, Nayarit, Nuevo León, Oaxaca, Puebla, Querétaro, Sinaloa y Sonora (2 especies cada una); Bambusoideae - Veracruz (6) y Chiapas (4); Chloridoideae - Chiapas, Nuevo León, Oaxaca (17),Veracruz (16); Chihuahua, Durango, San Luis Potosí (16), Durango (15); Danthonioideae - Baja California, Chihuahua, Sonora (3); Oryzoideae - Nayarit, Veracruz(2); Panicoideae - Veracruz (28), Campeche, Oaxaca (23), Chiapas, Jalisco (22), Yucatán (20); Pooideae - Baja California (33), Chihuahua, México(23). Las demás subfamilias no presentan especies cultivadas o introducidas: Anomochlooideae, Aristidoideae, Micrairoideae y Pharoideae.

Por su parte, se observa que entre 10 y $15 \%$ de los taxa registrados en las diferentes entidades federativas, son introducidos o cultivados (Tabla 3). En particular, las entidades que destacan en este rubro son Veracruz (67), Chiapas (65) y Oaxaca (65) (Tabla 3). Los porcentajes más altos de especies introducidas o cultivadas los presentan los estados peninsulares: Baja California (21\%), Campeche (19\%), Yucatán (22\%) y Quintana Roo (18\%). 
Avances en el estudio de las gramíneas. La actualización del conocimiento taxonómico de las gramíneas de México se realizó considerando más de 200 referencias bibliográficas publicadas en artículos científicos, floras, estudios florísticos y tesis publicados de 2006 a 2016. A partir de esta información se observa que hay varios grupos de trabajo enfocados al estudio filogenético de las diferentes subfamilias: Anomochlooideae y Aristidoideae (Strahan \& Allred 2008; Cerros-Tlatilpa \& Columbus 2009), Arundinoideae (Saltonstall \& Hauber 2007), Bambusoideae (Bamboo Phylogeny Group 2012, Kelchner \& Bamboo Phylogeny Group 2013, Chloridoideae (Peterson et al. 2007, 2010a,b, 2011, 2014a, 2015b, 2017a,b); Chemisquy et al. 2010, Snow et al. 2013), Danthonioideae (Baker et al. 2007, Reimer \& Cota-Sánchez 2007, Pirie et al. 2009, Linder et al. 2010), Micrairoideae (Sánchez-Ken et al. 2007), Oryzoideae (Martínez-y-Pérez 2006, Martínez-y-Pérez et al. 2006, 2008), Panicoideae (Soriano-Martínez et al. 2007, Zuloaga et al. 2007, 2010, Sede et al. 2009, Chemisquy et al. 2010, Morris \& Duvall 2010), Pharoideae (Sajo et al. 2007, Jones 2011), y Pooideae (Cialdella et al. 2007, 2014, Döring et al. 2007, Quintanar et al. 2007, Romaschenko et al. 2008, 2012, Schneider et al. 2009, 2012, RefulioRodriguez et al. 2012, Hochbach et al. 2015, Sclovich et al. 2015). Con base en los resultados de los estudios filogenéticos de las Poáceas, Soreng et al. (2015) proponen una nueva clasificación, que mejora el entendimiento de las relaciones genéricas y supragenéricas de los taxa. Por ejemplo, en la subfamilia Chloridoideae se encontraron, con el apoyo de las técnicas moleculares, géneros monofiléticos de reciente circunscripción como los siguientes: Distichlis (Bell \& Columbus 2008), que ahora incluye a Monanthochloe y Reederochloa; Muhlenbergia (Peterson et al. 2010b con la inclusión de 10 géneros (Aegopogon, Bealia, Blepharoneuron, Chaboissaea, Lycurus, Muhlenbergia, Pereilema, Redfieldia, Schaffnera, Schedonnardus) y Sporobolus (Peterson et al. 2014b, 2014c, 2017a) que incluye a Spartina. Por su parte, el género Kalinia fue segregado de Eragrostis (Bell et al. 2012).

Estudios de polifilia son también comunes en las Poáceas. Por ejemplo, el análisis de la subtribu Eleusininae (Peterson et al. 2015b), confirmó la polifilia del género Chloris, el cual fue separado en taxa monofiléticos y algunas de sus especies presentes en México fueron reclasificadas dentro de los géneros Tetrapogon (2 especies), Stapfochloa (2) y Leptochloa (3). Dentro de esta misma subtribu, se encontró que el género Leptochloa es de origen polifilético (Snow et al. 2013), mostrando 4 clados monofiléticos: Dinebra (6 especies), Diplachne (1), Disakisperma (1 ) y Leptochloa (ninguna especie mexicana).

Otro caso reciente en el que se proponen cambios en la clasificación de las Poáceas se refiere al reconocimiento de varias subtribus que incluyen cada una a Allolepis, Jouvea, Kalina y Sohnsia, que son géneros morfológica y genéticamente aislados (incertae sedis) dentro de la tribu Cynodonteae. Peterson et al. (2017b) consideran a estos taxa, filogenéticamente distintos a nivel de subtribus nuevas: Allolepiinae, Jouveinae, Kaliniinae y Sohnsiinae.

A partir de estos estudios, actualmente existen secuencias de nucleótidos para diferentes grupos de gramíneas que están almacenados en GenBank (NCBI 2016) y Barcode of Life Data System (Bold Systems 2014). Esta información se ha generado aplicando principalmente cuatro marcadores (ITS, rpL32-trnL, matK, y rbcL). Estos repositorios contienen secuencias de nucleótidos de al menos $50 \%$ de las especies mexicanas (aprox. 600), que son de acceso libre. Las especies mexicanas que ya fueron estudiadas filogenéticamente se distribuyen en Norteamérica y sus estudios moleculares fueron realizados en los Estados Unidos, en el marco del programa de Código de Barras de las gramíneas de esta región, que se llevó a cabo en el Instituto Smithsoniano y que dirigió Peterson et al. (2012 a 2014). De las especies mexicanas que no han sido analizadas filogenéticamente, se considera que es particularmente importante generar este conocimiento en los taxa endémicos a México o aquellos de distribución restringida

En lo que respecta al trabajo taxonómico, en los últimos 10 años se han realizado más de 60 trabajos monográficos o revisiones taxonómicas en cerca de $30 \%$ de los géneros mexicanos (Tabla 5). Aunque esto es un avance, sería muy relevante generar en los próximos 10 años, las revisiones taxonómicas de las restantes $(70 \%)$, con el fin de acercarse a la elaboración de la Flora de las Gramíneas de México.

En lo concerniente a los trabajos anatómicos y micromorfológicos, se estima que $35 \%$ de los géneros ha sido objeto de algún estudio. En los últimos años, destacan los siguientes estu- 
Tabla 5. Trabajos monográficos o revisiones taxonómicas que incluyen taxa mexicanos publicados en el periodo 2006-2016.

Subfamilia/género Referencia

Aristidoideae

Aristida

Strahan \& Allred 2008, Valdés-Reyna \& Allred 2010

Arundinoideae

Phragmites

Bambusoideae

Chusquea

Merostachys

Olmeca

Oteta

Saltonstall et al. 2004, Lambertini et al. 2006, Saltonstall \& Hauber 2007

Rhipidocladum

Chloridoideae

Bouteloua

Chloris

Ctenium, Gouinia

Cynodon, Micrachne

Leptochloa

Disakisperma

Distichlis

Gymnopogon

Kalinia

Muhlenbergia

Spartina

Sporobolus

Stapfochloa, Tetrapogon

Trichoneura, Triplasis

Ruíz-Sánchez \& Clark 2013, Ruíz-Sánchez et al. 2014a,b, Ruíz-Sánchez et al. 2015a

Ruíz-Sánchez et al. 2018

Ruíz-Sánchez et al. 2011a

Ruíz-Sánchez 2009, 2012, 2013, Ruíz-Sánchez et al. 2011b, Ruíz-Sánchez \& Castro-Castro 2016

Tyrrell 2008

\section{Danthonioideae}

Cortaderia, Danthonia

Oryzoideae

Luziola

\section{Panicoideae}

Aaica

Axonopus

Cenchrus

Dichanthelium

Digitaria

Hopia

Ocellochloa

Panicum

Paspalum

Schizachyrium

Stephostachys

Tripsacum

Siqueiros-Delgado 2007, Herrera-Arrieta et al. 2008, Halbrook 2012, Peterson et al. $2015 a$

Cerros-Tlatilpa et al. 2015, Peterson et al. 2015b

Peterson et al. 2014a

Peterson et al. 2015b

Peterson et al. 2012, Morales-Nieto et al. 2013

Snow et al. 2013

Bell \& Columbus 2008, López-Soto 2007, López-Soto et al. 2009

Cialdella \& Zuloaga 2011, Peterson et al. 2014a

Bell et al. 2012

Herrera-Arrieta \& Peterson 2007, Peterson et al. 2007, Giraldo-Cañas \& Peterson 2009

Kern et al. 2008, Mora-Olivo \& Valdés-Reyna 2011, Nieto-Silva et al. 2015

Denham \& Aliscioni 2011, Peterson et al. 2014c

Peterson et al. 2015b

Peterson et al. 2014a

Linder et al. 2010

Martínez-y-Pérez 2006, Martínez-y-Pérez et al. 2006, 2008

Grande-Allende 2014

Giraldo-Cañas 2008, 2012

Gutiérrez 2013

Hammer 2010

Sánchez-Ken 2012

Zuloaga et al. 2007

Sede et al. 2009

Sede et al. 2008, Soriano-Martínez 2010, Valdés-Reyna et al. 2009, Zuloaga et al. 2010

Denham et al. 2010, Ortíz-Díaz et al. 2015

Peichoto et al. 2008

Zuloaga et al. 2010

Vidal-Martínez et al. 2010 


\begin{tabular}{ll}
\hline Tabla 5. Continúa. & \\
\hline Subfamilia/género & Referencia \\
\hline Urochloa & Salariato et al. 2008 \\
Zea & Torres-Peña et al. 2015 \\
Zeugites & Soriano-Martínez et al. 2007, Soriano-Martínez 2008 \\
Zuloagaea & Bess et al. 2006 \\
Pooideae & \\
Agrostis & Zapiola et al. 2010, Dinler \& Budak 2008 \\
Avenella, Deschamsia, y & Chiapella \& Zuloaga 2010 \\
Vahlodea & \\
Briza, Chascolytrum & Essi et al. 2011 \\
Bromus & Saarela et al. 2014 \\
Elymus & Liu et al. 2006 \\
Glyceria & Whipple et al. 2007 \\
Hainardia, Parapholis & Schneider et al. 2012 \\
Jarava & Sclovich et al. 2015 \\
Dissanthelium & Refulio-Rodriguez 2007, Refulio-Rodriguez et al. 2012 \\
Poa & Soreng \& Peterson 2012 \\
Sphenopholis & Finot et al. 2004 \\
Trisetum & Finot et al. 2004, 2005 \\
\hline
\end{tabular}

dios: Reinheimer et al. (2005), Arriaga \& Barkworth (2006), Finot et al. (2006), Morrone et al. (2006), Liu et al. (2007), López-Soto (2007), Siqueiros-Delgado (2007), Kern et al. (2008), Salariato et al. (2008), Reinheimer \& Vegetti (2008), López-Soto et al. (2009), Reinheimer et al. (2009), Ingram (2010) y Zanotti et al. (2010). Prácticamente todos ellos han sido utilizados como complemento de los estudios filogenéticos y/o moleculares.

En cuanto a los trabajos florísticos, varios autores han contribuido con sus estudios en diferentes partes del país (Tabla 6), sobresaliendo los correspondientes a la región norte (Chihuahua, Coahuila y Durango) y a la zona central (Estado de México, Michoacán, Puebla y Veracruz). Entre ellos destacan: Levy-Tacher et al. (2006), López-Olmedo et al. (2006), Villarreal et al. (2006), Castillo-Campos et al. (2007), González-Costilla et al. (2007), Herrera-Arrieta \& Peterson (2007), Lira-Noriega et al. (2007), Paredes-Flores et al. (2007), Steinmann (2008a, b), Harker et al. (2008), Padilla-Velarde et al. (2008), Herrera-Arrieta \& Cortés-Ortíz (2009), Pulido-Esparza et al. (2009), Estrada-Castillón \& Villareal-Quintanilla (2010), Herrera-Arrieta \& Cortés-Ortíz (2010), Herrera-Arrieta et al. (2010), Ortíz-Díaz et al. (2010), Sánchez-Ken (2010), Soriano-Martínez (2010), Valdés-Reyna \& Allred (2010), Vidal-Martínez (2010 Sánchez-Ken (2011), Soriano-Martínez \& Dávila-Aranda (2011), González-Elizondo et al. (2012), Herrera-Arrieta et al. (2012), Lot (2012), Zepeda-Gómez et al. (2012), Cornejo-Tenorio et al. (2013), Garcillán et al. (2013), Burgos-Hernández et al. (2014), Hernández-Cárdenas et al. (2014), Herrera-Arrieta (2014), Londoño \& Ruíz-Sánchez (2014), Ruíz-Sánchez \& Clark (2014), Cabrera-Luna et al. (2015), León de la Luz et al. (2015), Ruíz-Sánchez et al. (2015b), Valdés-Reyna (2015), Valdés-Reyna et al. (2015), Vigosa-Mercado (2015), Morales-Arias et al. (2016), Vigosa-Mercado (2016), Vigosa-Mercado (2017a,b). La Figura 1, muestra un panorama general de la cobertura geográfica que han tenido estos estudios florísticos.

Especialistas y sus instituciones. A partir de la revisión bibliográfica y del conocimiento que tienen las autoras de los taxónomos activos que están trabajando algún grupo de la familia en México, se estima que hay aproximadamente 20 personas involucradas en el desarrollo de proyectos de investigación en Sistemática de la familia Poaceae. Estos taxónomos trabajan en las siguientes instituciones: 1) Colegio de Posgraduados (México); 2) Instituto de Ecología, A.C., sede Xalapa (Veracruz) y Centro Regional del Bajío (Michoacán); 3) Instituto Politécnico Na- 
Tabla 6. Trabajos florísticos en México realizados en el periodo 2006-2016

\begin{tabular}{|c|c|}
\hline Estado & Referencia bibliográfica \\
\hline Baja California & Garcillán et al. 2013, León de la Luz et al. 2015 \\
\hline Chiapas & Levy-Tacher et al. 2006 \\
\hline Chihuahua & $\begin{array}{l}\text { Herrera-Arrieta \& Peterson 2007, Herrera-Arrieta \& Cortés-Ortiz 2010, } \\
\text { Estrada-Castillón \& Villareal-Quintanilla } 2010\end{array}$ \\
\hline Coahuila & Villarreal et al. 2006, Valdés-Reyna 2015, Valdés-Reyna et al. 2015 \\
\hline Colima & Padilla-Velarde et al. 2008 \\
\hline Durango & $\begin{array}{l}\text { Herrera-Arrieta \& Cortés-Ortíz 2009, Herrera-Arrieta \& Cortés-Ortiz } \\
\text { 2010, Herrera-Arrieta et al. 2012, Herrera-Arrieta } 2014\end{array}$ \\
\hline Estado de México & Zepeda-Gómez et al. 2012 \\
\hline Guerrero & Vigosa-Mercado 2015, 2016, $2017^{a}$ \\
\hline Jalisco & Harker et al. 2008, Morales-Arias et al. 2016 \\
\hline Michoacán & $\begin{array}{l}\text { Steinmann 2008a,b, Cornejo-Tenorio et al. 2013, Cornejo-Tenorio, \& } \\
\text { Ibarra-Manríquez. 2017. Ruiz-Sanchez \& Clark 2014, Cabrera-Luna et } \\
\text { al. } 2015\end{array}$ \\
\hline Morelos & Pulido-Esparza et al. 2009, Hernández-Cárdenas et al. 2014 \\
\hline Nayarit & Vidal-Martínez et al. 2010, Ruiz-Sanchez \& Castro-Castro 2016 \\
\hline Oaxaca & López-Olmedo et al. 2006, Meave et al. 2017 \\
\hline Puebla & Paredes-Flores et al. 2007, Sánchez-Ken 2011, Vigosa-Mercado 2017b \\
\hline San Luis Potosí & González-Costilla et al. 2007 \\
\hline Veracruz & $\begin{array}{l}\text { Castillo-Campos et al. 2007, Lira-Noriega et al. 2007, Soriano- } \\
\text { Martínez 2010, Valdés-Reyna \& Allred 2010, Soriano-Martínez \& } \\
\text { Dávila 2011, Burgos-Hernández et al. 2014, Londoño \& Ruiz-Sanchez } \\
\text { 2014, Ruiz-Sanchez et al. 2015a }\end{array}$ \\
\hline Zacatecas & $\begin{array}{l}\text { Herrera-Arrieta \& Cortés-Ortiz 2010, Herrera-Arrieta et al. 2010, } \\
\text { Herrera-Arrieta et al. } 2012\end{array}$ \\
\hline $\begin{array}{l}\text { Península de Yucatán (Campeche, } \\
\text { Quintan Roo y Yucatán) }\end{array}$ & Ortíz-Díaz et al. 2010 \\
\hline
\end{tabular}

cional, Centro Interdisciplinario de Investigación para el Desarrollo Integral Regional. Unidad Durango (Durango), Escuela Nacional de Ciencias Biológicas (Ciudad de México) e Instituto Tecnológico de Chetumal (Quintana Roo); 4) Universidad Autónoma de Aguascalientes, Centro de Ciencias Básicas (Aguascalientes); 5) Universidad Autónoma Agraria Antonio Narro (Coahuila); 6) Universidad Autónoma de Chihuahua, Facultad de Zootecnia y Ecología (Chihuahua); 7) Universidad Autónoma del Estado de Hidalgo, Centro de Investigaciones Biológicas (Hidalgo); 8) Universidad Autónoma de Querétaro, Facultad de Ciencias Naturales (Querétaro); 9) Universidad Autónoma del Estado de Morelos (Morelos); 10) Universidad de Guadalajara, Departamento de Botánica y Zoología, CUCBA (Jalisco); 11) Universidad Autónoma de Yucatán, Campus de Ciencias Biológicas y Agropecuarias (Yucatán); 12) Universidad Nacional Autónoma de México, Facultad de Ciencias (Ciudad de México), Facultad de Estudios Superiores Iztacala (Estado de México), Facultad de Estudios Superiores Zaragoza (Ciudad de México) y, 13) Secretaría de Agricultura y Recursos Hidráulicos, Comisión Técnico Consultiva de Coeficientes de Agostadero (Ciudad de México), Rancho La Campana (Chihuahua).

\section{Discusión}

Soreng et al. (2015) mencionan la existencia en el planeta de 12 subfamilias, 771 géneros y 12,074 especies de la familia Poaceae. La única subfamilia que no está presente en México es Puelioideae, a la cual pertenecen los géneros Puelia y Guadella, que se distribuyen en África 
(Soreng et al. 2015). Asimismo, en el país se cuenta con $26.5 \%$ de los géneros y $10 \%$ de las especies reconocidas a nivel mundial.

Desde el 2006, cuando se publicó el Catálogo de las Gramíneas de México, no se había actualizado la lista de las gramíneas mexicanas, mostrando los cambios en su clasificación. En 2016, Villaseñor presenta una lista que, en comparación con los datos generados en este trabajo, presenta una diferencia numérica de 304 especies, que representan $25 \%$ de las especies incluidas en el presente estudio. Sin embargo, de éstas, $10 \%$ corresponde a especies introducidas, las cuales no fueron consideradas por Villaseñor. Por lo tanto, la discrepancia en ambos trabajos sería de $15 \%$ de las especies (182) y se debe a los cambios que recientemente han ocurrido en la clasificación de la familia, en particular, en la publicación de especies nuevas, cambios nomenclaturales y la segregación de géneros tales como Dinebra, Diplachne, Hyparrhenia, Lachnagrostis, Morronea, Stenotaphrum, Stephostachys, Thinopyrum y Trichanthecium.

Cuando de gramíneas se trata, es común considerarlas como plantas ruderales o malezoides, que prácticamente se establecen y desarrollan en múltiples hábitats. Sin embargo, se ha demostrado que su distribución está asociada a las regiones climáticas y altitudinales del país, como consecuencia de sus características anatómicas, bioquímicas y fisiológicas particulares (Giraldo-Cañas 2014). De hecho, es interesante que Soreng et al. (2015) estiman que $41 \%$ de las especies de gramíneas tienen un metabolismo $\mathrm{C} 4$, lo que les permite vivir en hábitats cálidos. Sin embargo, también hay muchas especies de gramíneas que pueden establecerse en ambientes diversos y son las que normalmente actúan como malezas.

En el mismo contexto, se registraron 295 especies endémicas de México, lo que representa $24.3 \%$ de su riqueza total. Resalta el hecho de que $32.2 \%$ de estas especies (95) solamente se distribuye en un solo estado y $15 \%$ (44) se registra en dos estados. Sin embargo, en contraste con esta especificidad de patrones de distribución, también se registran 26 especies endémicas de México que se distribuyen en 10 o más estados.

Un elemento adicional en la composición florística de la familia Poaceae en México, lo representan los 154 taxa introducidos o cultivados. Resulta interesante que las entidades que poseen porcentajes más altos de especies introducidas o cultivadas son las peninsulares: Baja California (21 \%), Campeche (19\%), Yucatán (22\%) y Quintana Roo (18\%).

Algunos de estos taxa se han extendido por prácticamente todo el territorio mexicano, actuando como malezas, en ocasiones, muy agresivas. Resultaría muy importante un estudio integral que recopile las características taxonómicas, ecológicas y fisiológicas de los taxa introducidos más abundantes, con el fin de conocer el impacto de su presencia para las especies nativas del país en lo particular y de los ecosistemas en lo general.

En lo concerniente al estado actual del conocimiento de las gramíneas resalta la cantidad de trabajos de distinta índole que se han publicado en los últimos 10 años. En buena medida, estos trabajos han estado encaminados a delimitar e inferir la evolución y las relaciones filogenéticas de las familias, subfamilias, tribus, géneros y especies de la familia Poaceae (Tabla 5). También se han seguido realizando monografías o revisiones taxonómicas y se ha contribuido en diferentes trabajos florísticos dando cuenta de las floras agrostológicas regionales. Se considera que falta mucho por conocer en cada uno de estos ámbitos. Es importante seguir desarrollando trabajos filogenéticos y filogeográficos, combinando datos moleculares, morfológicos y biogeográficos, que nos acerquen al entendimiento integral de las especies y de su estado de conservación, por ejemplo de las especies endémicas, las útiles, las introducidas y las que actúan como malezas. Igualmente se requiere seguir generando información morfológica, anatómica, citológica y fitoquímica de las gramíneas mexicanas, especialmente las que se utilizan como forraje y las que se comportan como malezas. En particular, se considera importante enfatizar que en los próximos años habría que promover en el país, el trabajo conjunto de quienes trabajan en esta familia botánica, para elaborar la Flora Agrostológica de México. Para lograrlo se considera importante construir una organización de especialistas interesados en este trabajo, provenientes del país y del extranjero, a partir de la cual se puedan definir las tareas que se habrían de realizar y promover la colaboración y el intercambio de información.

Paralelamente se tendría que fortalecer el trabajo de campo bien planificado y dirigido a las regiones y áreas menos exploradas (Figura 1), tales como las alejadas de las principales ciudades o aquellas de difícil acceso, ya sea por lo escarpado del terreno o por problemas de seguri- 
dad, como ya ha sido referido por Cortés-Ortiz \&Herrera-Arrieta (2011). Estas exploraciones botánicas tendrían que diseñarse, a partir de la información de los ejemplares de herbario y de las bases de datos disponibles e incluir la participación de ecólogos que estuvieran interesados en documentar los requerimientos ecológicos y filogeográficos de algunas especies, principalmente las forrajeras, endémicas e introducidas, dada su importancia biológica, económica y social. También es evidente que hay muy pocas personas realizando trabajo sistemático de gramíneas en el país y por lo tanto, se requiere fomentar la formación de especialistas en esta área. Este proceso de formación, se podría potenciar a partir del proyecto de construcción de la Flora Agrostológica de México. Todas estas acciones requieren apoyo del estado y financiamiento, lo cual solamente podrá ser obtenido, con el trabajo integral de quienes estamos interesados en el estudio de la sistemática de la familia Poaceae.

\section{Agradecimientos}

Agradecemos a la Sociedad Botánica de México por habernos invitado a preparar y presentar el trabajo intitulado "Conocimiento taxonómico actual de la familia Poaceae en México", en el marco del XX Congreso Mexicano de Botánica, organizado por la Sociedad Botánica de México, A.C., efectuado en septiembre de 2016. A partir de esta invitación, se preparó este trabajo. También queremos agradecer a Flor Isela Retana Rentería, por la elaboración del mapa de distribución (Figura 1). Por último expresamos nuestra gratitud a los revisores, quienes con sus comentarios y críticas permitieron mejorar el manuscrito.

\section{Literatura citada}

Arriaga MO, Barkworth MA. 2006. Amelichloa: a new genus in the Stipeae (Poaceae). Sida 22: 145-149.

Baker NP, Galley C, Verboom GA, Mafa P, Gilbert M, Linder HP. 2007. The phylogeny of the austral grass subfamily Danthonioidea: Evidence from multiple data sets. Plant Systematics and Evolution 264: 135-156. DOI 10.1007/s00606-006-0479-9

Bamboo Phylogeny Group . 2012. An updated tribal and subtribal classification of the Bamboos (Poaceae: Bambusoideae). Bamboo Science and Culture 24: 1-10. DOI 10.1600/036364416X694062

Bell HL, Columbus JT. 2008. Proposal for an expanded Distichlis (Poaceae, Chloridoideae): Support from molecular, morphological, and anatomical characters. Systematic Botany 33: 536- 551.

Bell HL, Columbus JT, Imgram, AL. 2012. Kalinia, a new North American genus for species long misplaced in Eragrostis (Poaceae, Chloridoideae). Aliso 30: 85-95. DOI: 10.5642/aliso.20133002.03

Bess EC, Doust AN, Davidse G, Kellogg EA. 2006. Zuloagaea, a new genus of neotropical grass within the "Bristle Clade" (Poaceae: Paniceae). Systematic Botany 31: 656- 670. DOI 10.1600/03636440677 9695898

BOLD Systems. 2014. Barcode of Life Data System V4. www.boldsystems.org (accessed April, 2016)

Burgos-Hernández M, Castillo-Campos G, Vergara-Tenorio MC. 2014. Potentially useful flora from the tropical rainforest in central Veracruz, Mexico: considerations for their conservation. Acta Botanica Mexicana 109: 55-77.

Cabrera-Luna JA, Huerta-Cantera HE, Salinas-Soto P, Olvera-Valerio D. 2015. Flora y vegetación de la sierra El Rincón, Querétaro y Michoacán, México. Botanical Sciences 93: 615-632. DOI 10.17129/ botsci. 168

Castillo-Campos G, Dávila-Aranda P, Zavala-Hurtado JA. 2007.La selva baja caducifolia en una corriente de lava volcánica en el centro de Veracruz: lista florística de la flora vascular. Boletín de la Sociedad Botánica de México 80: 77-104. DOI 10.17129/botsci.1747

Cerros-Tlatilpa R, Columbus JT. 2009. C Photosynthesis in Aristida longifolia: Implications for photosynthetic diversification in Aristoideae (Poaceae). American Journal of Botany 96: 1379-1387. DOI 10.3732/ajb.0800265

Cerros-Tlatilpa R, Siquieros-Delgado ME, Skendzic EM. 2015. El género Chloris Sw. (Poaceae: Chloridoideae) en México. Acta Botanica Mexicana 112: 95-147. DOI 10.21829/abm112.2015.1091

Chemisquy MA, Giussani LM, Scataglini MA, Kellogg EA, Morrone O. 2010. Phylogenetic studies favour the unification of Pennisetum, Cenchrus and Odontelytrum (Poaceae): a combined nuclear, plastid and morphological analysis, and nomenclatural combinations in Cenchrus. Annals of Botany 106: 107130. DOI 10.1093/aob/mcq090

Chiapella J, Zuloaga FO. 2010. A revision of Deschampsia, Avenella, and Vahlodea (Poaceae, Poeae, Airinae) in South America. Annals of the Missouri Botanical Garden 97: 141-162. DOI 10.3417/2008115 
Cialdella AM, Giussani LM, Aagesen L, Zuloaga FO, Morrone O. 2007. A phylogeny of Piptochaetium (Poaceae: Stipeae) and related genera based on a combined analysis including trnL-F, rpl16, and morphology. Systematic Botany 32: 545-559. DOI 10.1600/036364407782250607

Cialdella AM, Zuloaga FO. 2011. Taxonomic study of Gymnopogon (Poaceae, Chloridoideae, Cynodonteae). Annals of the Missouri Botanical Garden 98: 301-330. DOI 10.3417/2009071

Cialdella AM, Sede SM, Romaschenko K, Peterson PM, Soreng RJ, Zuloaga FO, Morrone O. 2014. Phylogeny of Nassella (Stipeae, Pooideae, Poaceae). Based on analyses of chloroplast and nuclear ribosomal DNA and morphology. BioOne 39: 814-828. DOI 10.1600/036364414X681419

CONABIO [Comisión Nacional para el Conocimiento y Uso de la Biodiversidad ]. 2016. Red Mundial de Información sobre Biodiversidad (REMIB). http://www.conabio.gob.mx/remib/doctos/remib_esp. html (accessed February-August, 2016)

CONABIO. 2017. Red Mundial de Información sobre Biodiversidad (REMIB). http://www.conabio.gob. $\mathrm{mx} /$ remib/doctos/remib_esp.html (accessed September, 2017)

Cornejo-Tenorio G, Sánchez-García E, Flores-Tolentino M, Santana-Michel FJ, Ibarra- Manríquez G. 2013. Flora y vegetación del cerro El Águila, Michoacán, México. Botanical Sciences 91: 155-180. DOI: $10.17129 /$ botsci.411

Cornejo-Tenorio G, Ibarra-Manríquez G. 2017. Flora of the core areas in the Monarch Butterfly Biosphere Reserve, Mexico: composition, geographical affinities and beta diversity. Botanical Sciences 95: 103 129. DOI 10.17129botsci.803

Cortés-Ortiz A, Herrera-Arrieta Y. 2011. Distribución y Diversidad de la familia Poaceae en Chihuahua, Durango y Zacatecas, México. Journal of the Botanical Research Institute of Texas 5: 689-700.

Dávila AP, Mejía-Saulés MT, Gómez-Sánchez M, Valdés-Reyna J, Ortíz JJ, Morín C, Castrejón J, Ocampo A. 2006. Catálogo de Gramíneas de México. México, D.F.: Universidad Nacional Autónoma de México/Comisión Nacional para el Conocimiento y Uso de la Biodiversidad. ISBN: 9703236707

Denham SS, Morrone O, Zuloaga FO. 2010. Estudios en el género Paspalum (Poaceae, Panicoideae, Paniceae): Paspalum denticulatum y especies afines. Annals of the Missouri Botanical Garden 97: 11-33. DOI $10.3417 / 2008092$

Denham SS, Aliscioni SS. 2011. El complejo Sporobolus indicus (Poaceae, Chloridoideae, Zoysieae) en la Argentina. Darwiniana 49: 32-42. DOI 10.2307/23230233

Dinler G, Budak, H. 2008. Analysis of expressed sequence Tags (ESTs) from Agrostis species obtained using sequence related amplified polymorphism. Biochemical Genetics 46: 663-676. DOI 10.1007/ s10528-008-9181-7

Döring E, Schneider J, Hilu KW, Röser M. 2007. Phylogenetic relationships in the Aveneae/Poeae complex (Pooideae, Poaceae). Kew Bulletin 62: 407-424.

Essi L, Wagner HML, de Souza Chies TT. 2011. New combinations within the Briza complex Poaceae, Pooideae, Poeae). Novon 21: 326-330. DOI 10.3417/2010026

Estrada-Castillón E, Villarreal-Quintanilla JA. 2010. Flora del centro del Estado de Chihuahua, México. Acta Botanica Mexicana 92: 51-118. DOI 10.21829/abm92.2010.283

Finot VL, Peterson PM, Soreng RJ, Zuloaga FO. 2004. A revision of Trisetum, Peyritschia, and Sphenopholis (Poaceae: Pooideae: Aveninae) in Mexico and Central America. Annals of the Missouri Botanical Garden 91: 1-30.

Finot VL, Peterson PM, Soreng RJ, Zuloaga FO. 2005. A revisión of Trisetum and Graphephorum (Poaceae: Pooideae: Aveninae) in North America North of Mexico. Sida 21: 1419-1453.

Finot VL, Baeza CM, Matthei O. 2006. Micromorfología de la epidermis de la lemma de Trisetum y géneros afines (Poaceae, Pooideae). Darwiniana 44: 32-57.

Garcillán PP, León de la Luz JL, Rebman JP, Delgadillo J. 2013. Plantas no nativas naturalizadas de la Península de Baja California, México. Botanical Sciences 91: 461-475. DOI 10.17129/botsci.423

NCBI [International Center for Biotechnology Information]. 2016. GenBank. http://www.ncbi.nlm.nih. gov/genbank (accessed April-June, 2016)

Giraldo-Cañas D. 2008. Revisión del género Axonopus (Poaceae: Paniceae): primer registro del género en Europa y novedades taxonómicas. Caldasia 30: 301-314.

Giraldo-Cañas D. 2012. Las especies del género Axonopus (Poaceae: Panicoideae: Paspaleae) en México. Caldasia 34: 325-346.

Giraldo-Cañas D. 2014. Riqueza y distribución altitudinal de Gramíneas C3 y C4 en la Guayana Venezolana. Revista Ciencia en Desarrollo 5: 77-84. DOI 10.19053/01217488.3233

Giraldo-Cañas D, Peterson PM. 2009. El género Muhlenbergia (Poaceae: Chloridoideae: Cynodonteae: Muhlenbergiinae) en Colombia. Caldasia 31: 269-302.

González-Costilla, Giménez de Azcárate J, García-Pérez J, Aguirre-Rivera JR. 2007. Flórula vascular de la Sierra de Catorce y territorios adyacentes, San Luis Potosí, México. Acta Botanica Mexicana 78: 1-38. DOI 10.21829/abm78.2007.1027 
González-Elizondo M, González-Elizondo M, Tena-Flores JA, Ruacho-González L, López-Enríquez L. 2012. Vegetación de la Sierra Madre Occidental, México: una síntesis. Acta Botanica Mexicana 100: 351-405. DOI 10.21829/abm100.2012.40

Grande-Allende JR. 2014. Additional segregates from Panicum incertae sedis. Phytoneuron 22: 1-6.

Gutiérrez HF. 2013. Estudios de las Especies Americanas del Género Cenchrus L. (Poaceae: Panicoideae: Paniceae). PhD Thesis, Universidad Nacional del Litoral. Santa Fe, Argentina.

Halbrook AK. 2012. Bouteloua curtipendula (Poaceae): Reproductive Biology, Phenotypic Plasticity, and the Origins of an Apomictic Species Complex. PhD. Thesis, University of Arizona.

Hammer RL. 2010. Systematic and evolutionary studies in the Dichanthelium acuminatum (Poaceae: Paniceae) complex. PhD Thesis, Texas A\&M University.

Harker M, García-Rubio LA, Riojas-López ME. 2008. Composición florística de cuatro hábitats en el rancho Las Papas de Arriba, municipio de Ojuelos de Jalisco, Jalisco, México. Acta Botanica Mexicana 85: 1-29. DOI 10.21829/abm85.2008.1069

Hernández-Cárdenas RA, Cerros-Tlatilpa R, Flores-Morales A. 2014. Las plantas vasculares y vegetación de la barranca Tepecapa en el municipio de Tlayacapan, Morelos, México. Acta Botanica Mexicana 108: 11-38. DOI 10.21829/abm108.2014.200

Herrera-Arrieta Y. 2014. Additions and updated names for grasses of Durango, Mexico. Acta Botanica Mexicana 106: 79-95. DOI: 10.21829/abm106.2014.214

Herrera-Arrieta Y, Peterson PM. 2007. Muhlenbergia (Poaceae) de Chihuahua, México. Sida Botanical Miscellany 29: 1-109. ISBN: 1-889878-16-2

Herrera-Arrieta Y, Peterson PM, Valdés-Reyna J. 2008. Bouteloua (Poaceae: Chloridoideae: Cynodonteae: Boutelouinae) del noreste de México. Journal of the Botanical Research Institute of Texas 2: 917-981.

Herrera-Arrieta Y, Cortés-Ortíz A. 2009. Diversidad de las Gramíneas de Durango, México. Polibotánica 28: 49-68.

Herrera-Arrieta Y, Cortés-Ortíz A. 2010. Listado florístico y aspectos ecológicos de la familia Poaceae para Chihuahua, Durango y Zacatecas, México. Journal of the Botanical Research Institute of Texas 4: 711-738.

Herrera-Arrieta Y, Peterson PM, Cortés-Ortíz A. 2010. Gramíneas de Zacatecas, México. Sida Botanical Miscellany 32: 1-239. ISBN 13 978-1889878-31-7

Herrera-Arrieta Y, Silva-Salas CA, Ruacho-González L, Rosales-Carrillo O. 2012. Nuevos registros de Poáceas para el norte de México. Journal of the Botanical Research Institute of Texas 6: 583-586.

Hochbach A, Schneider J, Röser M. 2015. A multi-locus analysis of phylogenetic relationships within grass subfamily Pooideae (Poaceae) inferred from sequences of nuclear single copy gene regions compared with plastid DNA. Molecular Phylogenetics and Evolution 87: 14-27. DOI 10.1016/ j.ympev.2015.03.010

Ingram AL. 2010. Evolution of leaf blade anatomy in Eragrostis (Poaceae). Systematic Botany 35: $755-$ 765. DOI 10.1600/036364410X539844

Jones SS. 2011. The Complete Chloroplast Genome of Pharus latifolius (Pharoideae; Poaceae) and Implications on the Early Evolution of the Grasses. MSc Thesis, Northern Illinois University.

Kelchner SA, Bamboo Phylogeny Group. 2013. Higher level phylogenetic relationships within the bamboos (Poaceae: Bambusoideae) based on five plastid markers. Molecular Phylogenetics and Evolution 67: 404-413. DOI 10.1016/j.ympev.2013.02.005

Kern VG, Guarise NJ, Vegetti AC. 2008. Inflorescence structure in species of Spartina Schreb. (Poaceae: Chloridoideae: Cynodonteae). Plant Systematics and Evolution 273: 51-61. DOI 10.1007/s00606-0080009-z

Lambertini C, Gustafsson MHG, Frydenberg J, Lissner J, Speranza M, Brix H. 2006. A phylogeographic study of the cosmopolitan genus Phragmites (Poaceae) based on AFLPs. Plant Systematics and Evolution 258: 161-182. DOI 10.1007/s00606-006-0412-2

León de la Luz JL, Medel-Narváez A, Domínguez-Cadena R. 2015. Floristic diversity and notes on the vegetation of Bahía Magdalena area, Baja California Sur, México. Botanical Sciences 93: 579-600. DOI: $10.17129 /$ botsci.159

Levy-Tacher SI, Aguirre-Rivera JR, García-Pérez JD, Martínez-Romero MM. 2006. Aspectos florísticos de Lacanhá Chansayab, Selva Lacandona, Chiapas. Acta Botanica Mexicana 77: 69-98.

Linder HP, Baeza M, Barker NP, Galley C, Humphreys AM, Lloyd KM, Orlovich DA, Pirie MD, Simon BK, Walsh N, Verboom GA. 2010. A generic classification of the Danthonioideae (Poaceae). Annals of Missouri Botanical Garden 97: 306-364. DOI 10.3417/2009006

Lira-Noriega A, Guevara S, Laborde J, Sánchez-Ríos G. 2007. Composición florística en potreros de Los Tuxtlas, Veracruz, México. Acta Botanica Mexicana 80: 59-87. DOI 10.21829/abm80.2007.1047

Liu Q, Ge S, Tang H, Zhang X, Zhu G, Lu BR. 2006. Phylogenetic relationships in Elymus (Poaceae: 
Triticeae) based on the nuclear ribosomal internal transcribed spacer and chloroplast trnL-F sequences. The New Phytologist 170: 411-420. DOI 10.1111/j.1469-8137.2006.01665.x

Liu Q, Peterson PM, Columbus JT, Zhao N, Hao G, Zhang D. 2007. Inflorescence diversification in the "Finger Millet Clade" (Chloridoideae, Poaceae): a comparison of molecular phylogeny and developmental morphology. American Journal of Botany 94: 1230-1247. DOI 10.3732/ajb.94.7.1230

Londoño X, Ruíz-Sánchez E. 2014. Guadua tuxtlensis (Poaceae: Bambusoideae: Bambuseae: Guaduinae), una nueva especie inadvertida de la región de Los Tuxtlas, Veracruz, México. Botanical Sciences 92: 481-488. DOI 10.17129/botsci.76

López-Olmedo LI, Pérez-García EA, Meave JA. 2006. Estructura y composición florística de las sabanas de la región de Nnizanda, Iistmo de Tethuantepec (Oaxaca), México. Acta Botanica Mexicana 77: 41-67.

López-Soto MM. 2007. Anatomía sistemática de la lámina foliar del géneros Distichlis (Poaceae). MsC. Thesis, Colegio de Postgraduados. Ciencias Agrícolas, Montecillos.

López-Soto MM, Koch KS, Flores-Cruz M, Engleman EM. 2009. Anatomía comparada de la lámina foliar del género Distichlis (Poaceae). Acta Botanica Mexicana 89: 1-23.

Lot A. 2012. Las Monocotiledóneas acuáticas y subacuáticas de México. Acta Botanica Mexicana 100: 135-148.

Martínez-y-Pérez JL. 2006. Relaciones Filogenéticas y Variaciones Morfológicas del Género Americano Luziola (Poaceae: Oryzeae). PhD Thesis. Instituto de Ecología A.C.

Martínez-y-Pérez JL, Sosa V, Mejía-Saulés T. 2006. Species delimitation in the Luziola peruviana (Poaceae) complex. Brittonia 58: 362-375. DOI 10.1663/ooo7-196X(2006)58[362:SDITLP]2.0.CO;2

Martínez-y-Pérez JL, Mejía-Saulés T, Sosa V. 2008. A taxonomic revision of Luziola (Poaceae: Oryzeae). Systematic Botany 33: 702-718. DOI 10.1600/036364408786500226

Meave JA, Rincón-Gutiérrez A, Ibarra-Manríquez G, Gallardo-Hernández C, \& Romero-Romero MA. 2017. Checklist of the vascular flora of a portion of the hyper-humid region of La Chinantla, Northern Oaxaca Range, Mexico. Botanical Sciences 95: 722-759. DOI 10.17129/botsci.1812

Mora-Olivo A, Valdés-Reyna J. 2011. Nota sobre la presencia de Spartina alterniflora Loisel. (Poaceae: Chloridoideae: Cynodonteae) en humedales mexicanos. Acta Botanica Mexicana 95: 45-50. DOI 10.21829/abm95.2011.266

Morales-Arias JG, Cuevas-Guzmán R, Rodríguez-Hernández JL, Guzmán-Hernández L, Núñez-López NM, Sánchez-Rodríguez EV, Solís-Magallanes A, Santana-Michel FJ. 2016. Flora vascular de Villas de Cacoma, sierra de Cacoma, Jalisco, México. Botanical Sciences 94: 393-418. DOI 10.17129/botsci. 447

Morales-Nieto CR, Rivero-Hernández O, Melgoza-Castillo A, Jurado-Guerra P, Martínez-Salvador M. 2013. Caracterización morfológica y molecular de Leptochloa dubia (Poaceae) en Chihuahua, México. Polibotánica 36: 79-94.

Morris LM, Duvall MR. 2010. The chloroplast genome of Anomochloa marantoidea Anomochlooideae: Poaceae) comprises a mixture of grass-like and unique features. American Journal of Botany 97: 620627. DOI 10.3732/ajb.0900226

Morrone O, Escobar A, Zuloaga FO. 2006. Chromosome studies in American Panicoideae (Poaceae). Annals of the Missouri Botanical Garden 93: 647-657. DOI 10.3417/0026-6493(2006)93[647: CSIAPP]2.0.CO;2

Nieto-Silva GR, Siqueiros-Delgado ME, Luna-Ruíz JJ, Flores-Ancira E, Moreno-Rico O. 2015. Taxonomía y distribución del género Spartina (Poaceae) en México. Botanical Sciences 93: 829-843. DOI $10.17129 /$ botsci. 85

Ortíz-Díaz JJ, Tun-Garrido J, Toledo-Hernández M. 2010. Diversidad de gramíneas en la Península de Yucatán. Bioagrociencias 3: 22-27.

Ortíz-Díaz JJ, Arnelas I, Cerros-Tlatilpa R, Siqueiros-Delgado ME, Tun-Garrido J. 2015. El género Paspalum L. (Paspaleae, Poaceae) en la Península de Yucatán, México. Acta Botanica Mexicana 113: 35-73. DOI: 10.21829/abm1 13.2015.1095

Padilla-Velarde E, Cuevas-Guzmán R, Koch SD. 2008. Plantas vasculares y vegetación de la parte alta del Arroyo Agua Fría, municipio de Minatitlán, Colima, México. Acta Botanica Mexicana 84: 25-72. DOI: $10.21829 / \mathrm{abm} 84.2008 .1066$

Paredes-Flores M, Lira-Sade R, Dávila-Aranda P. 2007. Estudio etnobotánico de Zapotitlán Salinas, Puebla. Acta Botanica Mexicana 79: 13-61. DOI: 10.21829/abm79.2007.1037.

Peichoto MC, Mazza SM, Solis VGN. 2008. Morphometric analysis of Schizachyrium condensatum (Poaceae) and related species. Plant Systematic and Evolution 276: 177-189. DOI 10.1007/s00606008-0076-1

Peterson PM, Valdés-Reyna J, Herrera-Arrieta Y. 2007. Muhlenbergiinae (Poaceae: Chloridoideae: Cynodonteae): from northeastern México. Journal of the Botanical Research Institute of Texas 1: 933-1000. 
Peterson PM, Romaschenko K, Johnson G.2010a. A phylogeny and classification of the Muhlenbergiinae (Poaceae: Chloridoideae: Cynodonteae) based on plastid and nuclear DNA sequences. American Journal of Botany 97: 1532-1554. DOI 10.3732/ajb.0900359

Peterson PM, Romaschenko K, Johnson G. 2010b. A classification of the Chloridoideae (Poaceae) based on multi-gene phylogenetic trees. Molecular Phylogenetics and Evolution 55: 580-598. DOI 10.1016/ j.ympev.2010.01.018

Peterson PM, Romaschenko K, Barker NP, Linder HP. 2011. Centropodieae and Ellisochloa, a new tribe and genus in the Chloridoideae (Poaceae). Taxon 60: 1113-1122. DOI 10.5167/uzh-56196

Peterson PM, Romaschenko K, Snow N, Johnson G. 2012. A molecular phylogeny and classification of Leptochloa (Poaceae: Chloridoideae: Chlorideae) sensu lato and related genera. Annals of Botany 109: 1317-1329. DOI 10.1093/aob/mcs077

Peterson PM, Romaschenko K, Herrera-Arrieta Y. 2014a. A molecular phylogeny and classification of the Cteniinae, Farragininae, Gouiniinae, Gymnopogoninae, Perotidinae, and Trichoneurinae (Poaceae: Chloridoideae: Cynodonteae). Taxon 63: 275-286. DOI 10.12705/632.35

Peterson PM, Romaschenko K, Herrera-Arrieta Y, Saarela JM. 2014b. A molecular phylogeny and new subgeneric classification of Sporobolus (Poaceae: Chloridoideae: Sporobolinae). Taxon 63: 12121243. DOI $10.12705 / 636.19$

Peterson PM, Romaschenko K, Herrera-Arrieta Y, Saarela JM. 2014c. Proposal to conserve the name Sporobolus against Spartina, Crypsis, Ponceletia, and Heleochloa (Poaceae: Chloridoideae: Sporobolinae). Taxon 63: 1373-1374. DOI 10.12705/636.23

Peterson PM, Romaschenko K, Herrera-Arrieta Y. 2015a. Phylogeny and subgeneric classification of Bouteloua with a new species, $B$. herrera-arrietae (Poaceae: Chloridoideae: Cynodonteae: Boutelouinae). Journal of Systematic and Evolution 53: 351-366. DOI 10.1111/jse.12159

Peterson PM, Romaschenko K, Herrera-Arrieta Y. 2015b. A molecular phylogeny and classification of the Eleusininae with a new genus, Micrachne (Poaceae: Chloridoideae: Cynodonteae). Taxon 64: 445-467. DOI 10.12705/643.5

Peterson PM, Romaschenko K, Herrera-Arrieta Y, Saarela JM. 2017a. A molecular phylogeny of the subtribe Sporobolinae and a classification of the subfamily Chloridoideae (Poaceae). The New York Botanical Garden 118: 127-151. DOI 10.21135/893275341.003

Peterson PM, Romachenko K, Herrera-Arrieta Y. 2017b. Four new subtribes: Allolepiinae, Jouveinae, Kaliniinae, and Sohnsiinae in the Cynodonteae (Poaceae: Chloridoideae). Phytoneuron 44: 1-9.

Pirie MD, Humphreys AM, Barker NP, Linder HP. 2009. Reticulation, data combination, and inferring evolutionary history: an example from Danthonioideae (Poaceae). Systematic Biology 58: 612-628. DOI 10.1093/sys-bio/syp068

Pulido-Esparza VA, Espejo-Serna A, López-Ferrari AR. 2009. Las Monocotiledóneas nativas del corredor biológico Chichinautzin. Acta Botanica Mexicana 86: 9-38.

Quintanar A, Castroviejo S, Catalan P. 2007. Phylogeny of the tribe Aveneae (Pooideae, Poaceae). Inferred from plastid trnF-L and nuclear ITS sequences. 2007. American Journal of Botany 94: 1554-1569. DOI 10.3732/ajb.94.9.1554

Refulio-Rodriguez NF. 2007. Systematics of Dissanthelium (Poaceae: Pooideae). PhD Thesis, Claremont Graduate University.

Refulio-Rodriguez NF, Columbus JT, Gillespie LJ, Peterson PM, Soreng JR. 2012. Molecular phylogeny of Dissanthelium (Poaceae: Pooideae) and its taxonomic implications. Systematic Botany 37: 122-133. DOI 10.1600/036364412X616701

Reimer E, Cota-Sánchez JH. 2007. An SME survey of the leaf epidermis in Danthonioid grasses (Poaceae: Danthonioideae). Systematic Botany 32: 60-70.

Reinheimer R, Pozner R, Vegetti AC. 2005. Inflorescence, spikelet, and floral development in Panicum maximum and Urochloa plantaginea (Poaceae). American Journal of Botany 92: 565-575. DOI 10.3732/ajb.92.4.565

Reinheimer R, Vegetti AC. 2008. Inflorescence diversity and evolution in the PCK clade (Poaceae: Panicoideae: Paniceae). Plant Systematics and Evolution 275: 133-167. DOI 10.1007/s00606-008-0057-4

Reinheimer R, Zuloaga FO, Vegetti AC, Pozner R. 2009. Diversification of inflorescence development in the PCK clade (Poaceae: Panicoideae: Paniceae). American Journal of Botany 96: 549-564. DOI 10.3732/ajb.0800245

Romaschenko K, Peterson PM, Soreng RJ, Garcia-Jacas N, Futorna O, Susanna A. 2008. Molecular phylogenetic analysis of the American Stipeae (Poaceae) resolves Jarava sensu lato polyphyletic: evidence for a new genus, Pappostipa. Journal of the Botanical Research Institute of Texas 2: 165-192.

Romaschenko K, Peterson PM, Soreng RJ, Garcia-Jacas N, Futorna O, Susanna A. 2012. Systematics and evolution of the needle grass (Poaceae: Pooideae: Stipeae) based on analysis of multiple chloroplast loci, ITS, and lemma micromorphology. Taxon 61: 18-44. 
Ruíz-Sánchez E. 2009. Delimitación de Especies y Posición Filogenética del Género de Bambú Americano Otatea (Poaceae: Bambusoideae). PhD. Thesis. Instituto de Ecología, A.C.

Ruíz-Sánchez E. 2012. A new species of Otatea (Poaceae: Bambusoideae: Bambuseae) from Queretaro, Mexico. Acta Botanica Mexicana 99: 21-29.

Ruíz-Sánchez E. 2013. Otatea ramirezii (Poaceae: Bambusoideae: Bambuseae) flower description and the importance of the Mexican national living bamboo collection. Phytotaxa 150: 54-60. DOI 10.11646/ phytotaxa.150.1.4

Ruíz-Sánchez E, Sosa V, Mejia-Saules MT. 2011a. Molecular Phylogenetics of the Mesoamerican bamboo Olmeca (Poaceae: Bambusoideae): Implications for taxonomy. Taxon 60: 89-98.

Ruíz-Sánchez E, Sosa V, Mejia-Saules MT, Londoño X, Clark LG. 2011b. A taxonomic revisión of Otatea (Poaceae: Bambusoideae: Bambuseae) including four new species. Systematic Botany 36: 314-336. DOI 10.1600/036364411X569516

Ruíz-Sánchez E, Clark, LG. 2013. Two new species of Chusquea (Poaceae: Bambusoideae: Bambuseae) from Mexico, one of them morphologically unusual, and a key to the Mexican sections of Chusquea. Phytotaxa 92: 1-12. DOI 10.11646/phytotaxa.92.1.1

Ruíz-Sánchez E, Clark LG. 2014. Familia Gramineae (Subfamilia Bambusoideae). Flora del Bajío y de Regiones adyacentes, Fascículo 186. Pátzcuaro, Michoacán: Instituto de Ecología, A.C. Centro Regional del Bajío.

Ruíz-Sánchez E, Mejia-Saules T, Clark LG. 2014a. A new endangered species of Chusquea (Poaceae: Bambusoideae) from the Acatlan volcano in central Veracruz, Mexico, and keys to the Mexican Chusquea species. Phytotaxa 163: 16-26. DOI 10.11646/phytotaxa.163.1.2

Ruíz-Sánchez E, Mejia-Saules T, Clark LG. 2014b. Chusquea nedjaquithii (Poaceae, Bambusoideae, Bambuseae, Chusquinae), a new endemic species from Oaxaca, Mexico. Phytotaxa 184: 23-30. DOI 10.11646/phytotaxa.184.1.3

Ruíz-Sánchez E, Mejia-Saules T, Cortes G, Clark LG. 2015a. Chusquea gibcooperi (Poaceae: Bambusoideae: Bambuseae: Chusqueinae), a new species endemic to Mexico. Brittonia 67: 227-232. DOI 10.1007/s12228-015-9377-1

Ruíz-Sánchez E, Clark LG, Londoño X, Mejia-Saules T, Cortes G. 2015b. Morphological keys to the genera and species of bamboos (Poaceae: Bambusoideae) of Mexico. Phytotaxa 236: 1-24. DOI 10.11646/phytotaxa.236.1.1

Ruíz-Sánchez E, Castro-Castro A. 2016. Otatea nayeeri (Poaceae: Bambusoideae: Bambuseae: Guaduinae), a new species endemic to Nayarit, Mexico. Phytotaxa 267: 211-218. DOI 10.11646/phytotaxa.267.3.4

Ruíz-Sánchez E, Clark, LG, Mejía-Saules, T, Lorea-Hernández, F. 2018. A new species of Merostachys (Poaceae: Bambudoideae: Bambuseae: Arthostylidiinae) with the northernmost distribution of the genus. Phytotaxa 344: 31-38. DOI 10.11646/phytotaxa.344.1.4

Saarela JM, Peterson PM, Valdés-Reyna J. 2014. A taxonomic revision of Bromus (Poaceae: Pooideae: Bromeae) in Mexico and Central America. Phytotaxa 185: 1-147. DOI 10.11646/phytotaxa.185.1.1

Sajo MG, Longhi-Wagner H, Rudall PJ. 2007. Floral development and embryology in the early -divergent grass Pharus. International Journal of Plant Sciences 168: 181-191. DOI: 10.1086/509790

Salariato DL, Morrone O, Zuloaga FO. 2008. Ornamentación del antecio superior en Urochloa y géneros vecinos (Poaceae, Panicoideae, Paniceae): su valor sistemático. Darwiniana 46: 335-355.

Saltonstall K, Peterson PM, Soreng RJ. 2004. Recognition of Phragmites australis subsp. americanus (Poaceae: Arundinoideae) in North America: evidence from morphological and genetic analyses. Sida 21: 683-692.

Saltonstall K, Hauber D. 2007. Notes on Phragmites australis (Poaceae: Arundinoideae) in North America. Journal of the Botanical Research Institute of Texas 1: 385-388.

Sánchez-Ken J. 2010. Two new species of Paspalum (Paniceae: Panicoideae: Poaceae), a preliminary checklist of the genus in Mexico, and the identity of P. crinitum. Revista Mexicana de Biodiversidad 81: 629-647.

Sánchez-Ken J. 2011. Poaceae. Flora del Valle de Tehuacán-Cuicatlán, Fascículo 81. Instituto deBiología, Universidad Nacional Autónoma de México. México, D.F.

Sánchez-Ken J.G. 2012. A synopsis of Digitaria (Paniceae, Panicoideae, Poaceae) in Mexico, including the new species Digitaria michoacanensis. Acta Botanica Mexicana 101: 127-149.

Sánchez-Ken J, Clark LG, Kellogg EA, Kay EE. 2007. Reinstatement and emendation of subfamily Micrairoideae (Poaceae). Systematic Botany 32: 71-80. DOI 10.1600/036364407780360102

Sánchez-Ken JG, Clark LG. 2010. Phylogeny and a new tribal classification of the Panicoideae s.1. (Poaceae) based on plastid and nuclear sequence data and structural data. American Journal of Botany 97: 1732-1748. DOI 10.3732/ajb.1000024

Schneider JG, Döring E, Hilu KW, Röser M. 2009. Phylogenetic structure of the grass Subfamily Pooi- 
deae based on comparison of plastid matK gene-3'trnK exon and nuclear ITS sequences. Taxon 58: 405-424.

Schneider J, Winterfeld G, Röser M. 2012. Polyphyly of the grass tribe Hainardieae (Poaceae: Pooideae): identification of its different lineages based on molecular phylogenetics, including morphological and cytogenetic characteristics. Organisms Diversity and Evolution 12: 113-132. DOI 10.1007/s13127012-0077-3

Sclovich SE, Giussani LM, Cialdella AM, Sede SM. 2015. Phylogenetic analysis of Jarava (Poaceae, Pooideae, Stipeae) and related genera: testing the value of the awn indumentum in the circumscription of Jarava. Plant Systematic and Evolution 301: 1625-1641. DOI 10.1007/s00606-014-1175-9

Sede SM, Morrone O, Giussani M, Zuloaga FO. 2008. Phylogenetic studies in the Paniceae (Poaceae): a realignment of Section Lorea of Panicum. Systematic Botany 33: 284- 300. DOI 10.1600/036364408 784571626

Sede SM, Zuloaga FO, Morrone O. 2009. Phylogenetic studies in the Paniceae (Poaceae- Panicoideae): Ocellochloa, a new genus from the new world. Systematic Botany 34: 684-692. DOI 10.1600/036364 409790139655

Siqueiros-Delgado ME. 2007. Culm anatomy of Bouteloua and relatives (Gramineae: Chloridoideae: Boutelouinae). Acta Botanica Mexicana 78: 39-59.

Snow N, Peterson PM, Romaschenko K. 2013. Systematics of Disakisperma (Poaceae, Chloridoideae, Chlorideae). PhytoKeys 26: 21-70. DOI 10.3897/phytokeys.26.5649

Soreng RJ, Peterson PM. 2012. Revision of Poa L. (Poaceae, Pooideae, Poeae, Poinae) in Mexico: new recods, re-evaluation of $P$. ruprechtii, and two new species, P. palmeri and P. wendtii. PhytoKeys 15: 1-104. DOI 10.3897/phytokeys.15.3084

Soreng RJ, Peterson PM, Romaschenko K, Davidse G, Zuloaga FO, Judziewicz EJ, Filgueiras TS, Davis JI, Morrone O. 2015. A worldwide phylogenetic classification of the Poaceae (Gramineae). Journal of Systematics and Evolution 53: 117-137. DOI 10.1111/jse.12150

Soriano-Martínez AM. 2008. Estudio Sistemático del Género Zeugites P. Browne (Poaceae: Centothecoideae: Centotheceae). PhD. Thesis, Universidad Nacional Autónoma de México.

Soriano-Martínez AM. 2010. Poaceae IV. Paniceae: Panicum. Flora de Veracruz, Fascículo 152. Xalapa, México. Instituto de Ecología, A.C.

Soriano-Martínez AM, Salazar GA, Dávila-Aranda P. 2007. Phylogenetic Relationships of Zeugites (Poaceae: Centothecoideae) Inferred from Plastid and Nuclear DNA Sequences and Morphology. Systematic Botany 32: 722-730. DOI 10.1043/06-75.1

Soriano-Martínez AM, Dávila-Aranda P. 2011. Poaceae V. Panicoideae: Tribu Centothecae. Flora de Veracruz, Fascículo 153. Xalapa, México. Instituto de Ecología A.C.

Steinmann VW. 2008a. Familia Gramineae (Subfamilia Ehrhartoideae). Flora del Bajío y de Regiones adyacentes, Fascículo 154. Pátzcuaro, Michoacán: Instituto de Ecología, A.C. Centro Regional del Bajío.

Steinmann VW. 2008b. Familia Gramineae (Subfamilia Arundinoideae). Flora del Bajío y de Regiones adyacentes. Fascículo 158. Pátzcuaro, Michoacán: Instituto de Ecología, A.C. Centro Regional del Bajío.

Strahan RT, Allred KW. 2008. Aristidae Eludendae II: A re-evaluation of the Aristida gibbosa complex (Poaceae: Aristideae), including A. marginalis, A. orizabensis, and A. sorzogonensis. Journal of the Botanical Research Institute of Texas 2: 309-322.

Thiers B. (con actualizaciones continuas). Index Herbarorium: A global directory of public herbaria and associated staff. New York Botanical Garden's Virtual Herbarium. $<$ http://sweetgum.nybg.org/science/ih/> (accessed January, 2018)

Torres-Peña G, De la Cruz-Larios L, Sánchez-González JJ, Ruíz-Corral JA, Castañeda-Nava JJ, Santacruz-Ruvalcaba F, Miranda-Medrano R. 2015. Relaciones entre poblaciones de teocintle (Zea spp.) de México, Guatemala y Nicaragua. Acta Botanica Mexicana 111: 17-45.

Tyrrell CD. 2008. Systematics of the Neotropical Woody Bamboo Genus Rhipidocladum (Poaceae: Bambusoidea). MSc.Thesis, Iowa State University.

Valdés-Reyna J. 2015. Gramíneas de Coahuila. México: Comisión Nacional para el Conocimietno y Uso de la Biodiversidad. ISBN: 978-607-8328-16-1

Valdés-Reyna J, Zuloaga FO, Morrone O, Aragón L. 2009. El género Panicum (Poaceae: Panicoideae) en el noreste de México. Boletín de la Sociedad Botánica de México 84: 59-82.

Valdés-Reyna J, Allred, KW. 2010. Poaceae III. Tribu Aristideae. Flora de Veracruz Fascículo 151. Xalapa, México: Instituto de Ecología, A.C..

Valdés-Reyna J, Villaseñor JL, Encina-Domínguez JA, Ortíz E. 2015. The grass family (Poaceae) in Coahuila, Mexico: Diversity and distribution. Botanical Sciences 93: 119-129. DOI 10.17129/botsci. 79 
Vidal-Martínez VA, Herrera-Cedano F, Coutiño-Estrada B, Sánchez-González JJ, Ron- Parra A, OrtegaCorona, Guerrero-Herrera MJ. 2010. Identificación y localización de una nueva especie de Tripsacum spp. en Nayarit, México. Revista Fitotecnia Mexicana 33: 27-30.

Vigosa-Mercado JL. 2015. Arundinoideae, Micrairoideae y Pharoideae (Poaceae). Flora de Guerrero No. 67. Universidad Nacional Autónoma de México, ISBN 978-607-02-7174-8

Vigosa-Mercado JL. 2016. Flora de Guerrero. Danthonioideae y Oryzoideae (Poaceae). Flora de Guerrero No. 72. Universidad Nacional Autónoma de México. ISBN 978-607-02-8516-5

Vigosa-Mercado JL. 2017a. Flora de Guerrero. Andropogoneae (Panicoideae, Poaceae). Flora de Guerrero No. 75. Universidad Nacional Autónoma de México, ISBN 978-607-02-9233-0

Vigosa-Mercado JL. 2017b. Pooideae (Poaceae). Flora del Valle de Tehuacán-Cuicatlán No. 168. Universidad Nacional Autónoma de México ISBN 978-607-02-9418-1

Villaseñor JL. 2016. Checklist of the native vascular plants of Mexico. Revista Mexicana de Biodiversidad 87: 559-902. DOI 10.1016/j.rmb.2016.06.017

Villarreal QJA, Carranza PMA, Estrada CE, Rodríguez GA. 2006. Flora riparia de los Ríos Sabinas y San Rodrigo, Coahuila, México. Acta Botanica Mexicana 75: 1-20.

Whipple IG, Barkworth ME, Bushman BS. 2007. Molecular insights into the taxonomy of Glyceria (Poaceae: Meliceae) in North America. American Journal of Botany 94: 551-557. DOI 10.3732/ ajb.94.4.551

Zanotti CA, Pozner R, Morrone O. 2010. Understanding spikelet orientation in Paniceae (Poaceae). American Journal of Botany 97: 717-729. DOI 10.3732/ajb.0900031

Zapiola ML, Cronn RC, Mallory-Smith CA. 2010. Development of novel chloroplast microsatellite markers to identify species in the Agrostis complex (Poaceae) and related genera. Molecular Ecology Resources 10: 738-740. DOI 10.1111/j.1755-0998.2009.02828.x

Zepeda-Gómez C, Lot-Helgueras A, Nemiga XA, Madrigal-Uribe D. 2012. Florística y diversidad de las Ciénegas del río Lerma Estado de México. Acta Botanica Mexicana 98: 23-49.

Zuloaga FO, Giussani LM, Morrone O. 2007. Hopia, a new monotypic genus segregated from Panicum (Poaceae). Taxon 56: 145-156.

Zuloaga FO, Scataglini MA, Morrone O. 2010. A phylogenetic evaluation of Panicum sects. Agrostoidea, Megista, Prionitia and Tenera (Panicoideae, Poaceae): Two new genera, Stephostachys and Sorengia. Taxon 59: 1535-1546. 
Apéndice 1. Gramíneas de México

Lista de especies de Poaceae de México. Para cada género se incluye entre paréntesis: total de especies /

total de especies endémicas de México presentes en dos o más estados / total de especies endémicas de México presentes en un solo estado / total de especies introducidas / subfamilia). La simbología utilizada en las especies y categorías infra específicas es la siguiente: * (endémicas de México presentes en 2 o más estados), ** (endémicas de México presentes en un solo estado) y + (introducida). Las abreviaturas de las entidades federativas son las siguientes: Aguascalientes (AGS), Baja California (BC), Baja California Sur (BCS), Campeche (CAM), Ciudad de México (CDM), Chiapas (CHIS), Chihuahua (CHIH), Coahuila (COAH), Colima (COL), Durango (DGO), Estado de México (MÉX), Guanajuato (GTO), Guerrero (GRO), Hidalgo (HGO), Jalisco (JAL), Michoacán (MICH), Morelos (MOR), Nayarit (NAY), Nuevo León (NL), Oaxaca (OAX), Puebla (PUE), Querétaro (QRO), Quintana Roo (QROO), San Luis Potosí (SLP), Sinaloa (SIN), Sonora (SON), Tabasco (TAB), Tamaulipas (TAM), Tlaxcala (TLX), Veracruz (VER), Yucatán (YUC), Zacatecas (ZAC).

Aakia (1/0/0/0; Panicoideae)

Aakia tuerckheimii (Hack.) J.R. Grande (CHIS, PUE, VER).

Achnatherum (13/3/2/0; Pooideae)

**Achnatherum acutum (Swallen) Valdés-Reyna \& Barkworth (COAH).

Achnatherum altum (Swallen) Hoge \& Barkworth (COAH).

**Achnatherum bracteatum (Swallen) Valdés-Reyna \& Barkworth (BC)

Achnatherum clandestinum (Hack.) Barkworth (AGS, COAH, MÉX, MICH, NL, PUE, SLP., ZAC).

*Achnatherum constrictum (Hitchc.) Valdés-Reyna \& Barkworth (HGO, MÉX).

Achnatherum coronatum (Thurb.) Barkworth (BC)

Achnatherum curvifolium (Swallen) Barkworth (CHIH).

*Achnatherum editorum (E. Fourn.) Valdés-Reyna ex Barkworth (COAH, HGO, NL, PUE, SLP., TAM, VER, ZAC).

Achnatherum eminens (Cav.) Barkworth (AGS, BC, CHIH, CHIS, COAH, DGO, GTO, HGO, JAL, MÉX, NL, OAX, PUE, QRO, SLP., SON, TAM, VER, ZAC).

Achnatherum hymenoides (Roem. \& Schult.) Barkworth (BC, $\mathrm{CHIH})$

*Achnatherum multinode (Scribn. ex Beal) Valdés-Reyna \& Barkworth (CHIH, ZAC).

Achnatherum parishii (Vasey) Barkworth, Phytologia 74(1): 11. 1993.

Achnatherum parishii subsp. depauperatum (M.E. Jones) Barkworth (COAH).

Achnatherum parishii (Vasey) Barkworth subsp. parishii (COAH, NL).

Achnatherum robustum (Vasey) Barkworth (CHIH).

Acroceras (1/0/0/0; Panicoideae)

Acroceras zizanioides (Kunth) Dandy (CAM, CHIS, OAX, PUE, SLP., TAB, VER, YUC).

+Aegilops (1/0/0/1; Pooideae)

+ Aegilops cylindrica Host $(\mathrm{CHIH})$.

+Agropyron (1/0/0/1; Pooideae)

+Agropyron cristatum (L.) Gaertn. (CHIH).

Agrostis (21/4/2/1; Pooideae)

*Agrostis bourgeaei E. Fourn. (CDM, GTO, HGO, MÉX, MICH, OAX, TLX).

**Agrostis calderoniae Acosta Cast. (MÉX).

Agrostis elliottiana Schult. (YUC).

Agrostis exarata Trin. (BC, CHIH, COAH, CDM, DGO, GTO, HGO).

Agrostis ghiesbreghtii E. Fourn. (CHIS, GRO, HGO, MÉX, MOR, OAX, PUE, VER).

+Agrostis gigantea Roth (MÉX, SLP).

Agrostis hyemalis (Walter) Britton, Sterns \& Poggenb. (MICH, QRO, VER).

Agrostis laxissima Swallen (CHIS).

*Agrostis liebmannii (E. Fourn.) Hitchc. (CDM, DGO, MÉX, OAX, PUE, QRO, VER).

Agrostis mertensii Trin. (MÉX).

Agrostis microphylla Steud. (BC).

${ }^{*}$ Agrostis novogaliciana McVaugh (JAL).

Agrostis pallens Trin. (BC).

Agrostis perennans (Walter) Tuck. (CHIS, CDM, DGO, GTO, HGO, MÉX, MICH, MOR, OAX, PUE, QRO, SLP, TLX, VER).

*Agrostis rosei Scribn. \& Merr. (CDM, DGO, ZAC).

Agrostis scabra Willd. (AGS, BC, CHIH, CHIS, COAH, DGO, GTO, JAL, MÉX, MICH, MOR, OAX, PUE,

QRO, S.L.P, VER).

*Agrostis schaffneri E. Fourn. (CHIS, CDM, HGO, JAL, MÉX, MICH, MOR, OAX, PUE, QRO, TLX, VER). 
Agrostis stolonifera L.

Agrostis stolonifera var. palustris (Huds.) Farw. (BC, CHIS, CDM, HGO, JAL, MÉX, PUE, TLX, VER). Agrostis subpatens Hitchc. (CHIS, CDM, HGO, JAL, MÉX, PUE, TLX, VER).

Agrostis tolucensis Kunth (CHIS, CDM, DGO, GTO, GRO, JAL, MÉX, MICH, MOR, OAX, PUE, TLX, VER). Agrostis virescens Kunth (CHIS, CDM, GRO, JAL, MÉX, MICH, MOR, OAX, PUE, TLX, VER).

Aira (1/0/0/1; Pooideae)

+Aira caryophyllea L. (BC, JAL, OAX).

Allolepis (1/0/0/0; Chloridoideae)

Allolepis texana (Vasey) Soderstr. \& H.F. Decker (COAH).

Alopecurus (2/0/0/0; Pooideae)

Alopecurus geniculatus L. $(\mathrm{CHIH})$.

Alopecurus saccatus Vasey (BC).

Andropogon (14/1/0/0; Panicoideae)

Andropogon angustatus (J. Presl) Steud. (CHIS, GRO, MÉX, NAY, OAX).

Andropogon bicornis L. (CAM, CHIS, GRO, HGO, JAL, MÉX, MICH, NAY, OAX, PUE, TAB, TAM, VER, YUC). Andropogon bourgeaei Hack. (CHIS, OAX, TAB, VER, YUC).

Andropogon fastigiatus Sw. CHIH, CHIS, GRO, JAL, MÉX, MICH, MOR, NAY, OAX, SIN, VER).

Andropogon gerardi Vitman

Andropogon gerardi Vitman var. gerardi (CHIS, COAH, DGO, GTO, MÉX, MICH, SIN).

Andropogon glomeratus (Walter) Britton, Sterns \& Poggenb.

Andropogon glomeratus (Walter) Britton var. glomeratus (BC, BCS, COL, SIN, SON).

Andropogon glomeratus var. pumilus (Vasey) Vasey ex L.H. Dewey (AGS, CAM, CHIH, CHIS, COAH,

DGO, GTO. GRO, HGO, JAL, MÉX, MICH, MOR, NL, OAX, PUE, QRO, QROO, SLP, TAB, TAM, TLX, VER, YUC, ZAC).

Andropogon gyrans Ashe (CHIS, PUE, SLP, VER).

Andropogon leucostachyus Kunth (CAM, CHIS, GRO, OAX, TAB, VER, YUC).

Andropogon liebmannii Hack. (CDM, HGO, JAL, MÉX, MICH, NAY, OAX, PUE, VER).

*Andropogon pringlei Scribn. \& Merr. (CHIS, CDM, DGO, JAL, MÉX, MICH, OAX, PUE, VER, ZAC).

Andropogon selloanus (Hack.) Hack. (CAM, CHIS, OAX, TAB, VER, YUC).

Andropogon ternarius Michx. (COAH).

Andropogon virgatus Desv. ex Ham. (CHIS).

Andropogon virginicus L. (CAM, CHIS, COAH, DGO, NL, OAX, PUE, QROO, TAB, VER, YUC).

Anthaenantia (2/0/1/0; Panicoideae)

Anthaenantia lanata (Kunth) Benth. (CAM, CHIS, COL, GRO., GTO., JAL, MÉX, MICH, NAY, OAX, PUE, QROO, SLP, TAB, TAM, VER, YUC).

**Anthaenantia villaregalis (McVaugh \& R. Guzmán) Espejo \& López-Ferrari (JAL).

Anthephora (1/0/0/0; Panicoideae)

Anthephora hermaphrodita (L.) Kuntze (BC, BCS, CAM, CHIS, COL, CDM, GRO, JAL, MÉX, MICH, MOR, NAY, OAX, PUE, QROO, SIN, SON, VER, YUC).

Anthoxanthum (1/0/0/1; Pooideae)

+Anthoxanthum odoratum L. (CHIH, CHIS, CDM, DGO, HGO, MÉX, OAX).

Aristida (33/8/5/0; Aristidoideae)

Aristida adscensionis L. (AGS, BC, BCS, CAM, CHIH, CHIS, COAH, COL, CDM, DGO, GTO, GRO, HGO, JAL, MÉX, MICH, MOR, NAY, NL, OAX, PUE, QRO, SLP, SIN, SON, TAM, VER, YUC, ZAC). Aristida appressa Vasey (CAM, CHIH, CHIS, CDM, DGO, GTO, JAL, MÉX, MICH, MOR, NAY, OAX, PUE, SLP, VER, YUC, ZAC).

Aristida arizonica Vasey (BC, CHIH, COAH, COAH, CDM, DGO, GTO, GRO, JAL, MÉX, MICH, MOR, NL, OAX, PUE, SLP, SIN, SON, TAM, ZAC).

Aristida californica Thurb.

Aristida californica var. glabrata Vasey (BC, BCS, SON).

Aristida capillacea Lam. (CHIS, COL, CDM, JAL, MÉX, NAY, OAX, SIN, VER).

Aristida divaricata Humb. \& Bonpl. ex Willd. (AGS, BC, CHIH, COAH, CDM, DGO, GTO, HGO, JAL, MÉX, MICH, NAY, NL, OAX, PUE, SLP, SON, TAM, TLX, VER, ZAC).

*Aristida eludens Allred \& Valdés-Reyna (CHIH, COAH, DGO, GTO, NL, OAX, QRO, SLP).

Aristida floridana (Chapm.) Vasey (CAM, QROO, YUC).

**Aristida geminiflora E. Fourn. (VER). 
Aristida gibbosa (Nees) Kunth (CAM, CHIH, CHIS, CDM, DGO, GTO, GRO, JAL, MÉX, MICH, NAY, OAX, PUE, SIN, SON, TLX, VER).

Aristida gypsophila Beetle

*Aristida gypsophila fo. diffusa Allred \& Valdés-Reyna (CHIH, SLP).

*Aristida gypsophila Beetle fo. gypsophila (COAH, SLP).

Aristida gypsophila fo. gypsophiloides Allred \& Valdés-Reyna (CHIH, COAH, SLP).

Aristida hamulosa Henrard (AGS, BC, CHIH, CHIS, CDM, DGO, GTO, JAL, MÉX, OAX, PUE, QRO, SLP, SON, VER, ZAC).

Aristida havardii Vasey (AGS, BC, CHIH, COAH, CDM, DGO, GTO, JAL, MÉX, NL, PUE, SLP, SON, VER, ZAC).

*Aristida hintonii Hitchc. (CDM, GRO, JAL, MÉX, MICH, NAY, OAX, TAM).

*Aristida hitchcockiana Henrard (GTO, JAL, NAY, OAX, PUE, QRO, ZAC).

*Aristida jaliscana R. Guzmán \& Jaramillo (GTO, JAL).

Aristida jorullensis Kunth (CAM, CHIS, COL, CDM, GRO, JAL, MÉX, MICH, NAY, OAX, PUE,QROO, SIN, SON, VER, YUC).

Aristida laxa Cav.

*Aristida laxa var. karwinskiana (Trin. \& Rupr.) Henrard (CDM, GTO, HGO, MÉX, MICH).

Aristida laxa Cav. var. laxa (CHIH, CDM, DGO, GTO, HGO, JAL, MÉX, MICH, MOR, NAY, OAX, PUE, SLP, SIN, SON, VER, ZAC).

Aristida laxa var. longiramea (J. Presl) Henrard (GRO, JAL, MICH, OAX).

Aristida liebmannii E. Fourn. (CHIS, OAX, VER).

Aristida longespica Poir. (CHIH, COAH, TAM, ZAC).

*Aristida mexicana Scribn. ex Henrard (AGS, CDM, GTO, JAL, MÉX, PUE).

Aristida pansa Wooton \& Standl.

*Aristida pansa fo. contracta Allred \& Valdés-Reyna (COAH, DGO, NL, TAM, ZAC).

Aristida pansa fo. dissita (I.M. Johnst.) Allred \& Valdés-Reyna (CHIH, COAH, NL, OAX, PUE, SLP, TAM, ZAC).

Aristida pansa Wooton \& Standl. fo. pansa (CHIH, COAH, COL, DGO, GTO, HGO, JAL, NL, OAX, PUE, QRO, SLP, TAM, ZAC).

**Aristida petersonii Allred \& Valdés-Reyna (OAX).

Aristida purpurascens Poir., Encycl. Suppl. 1: 452. 1810.

Aristida purpurascens var. tenuispica (Hitchc.) Allred (CHIS).

Aristida purpurea Nutt.

Aristida purpurea fo. brownii (Warnock) Allred \& Valdés-Reyna (AGS, BC, COAH, GTO, NL, PUE, SLP, TAM, VER, ZAC).

*Aristida purpurea var. curvifolia (E. Fourn.) Allred (AGS, COAH, DGO, NL, OAX, PUE, SLP, TAM, VER, ZAC).

Aristida purpurea var. fendleriana (Steud.) Vasey (BC, CHIH, COAH, NL, SLP, TAM, ZAC).

Aristida purpurea var. longiseta (Steud.) Vasey (AGS, BC, CHIH, COAH, DGO, GTO, NL, PUE, QRO, SLP, SON, TAM, VER, ZAC).

Aristida purpurea var. nealleyi (Vasey) Allred (AGS, BC, BCS, CHIH, COAH. DGO, GTO, NL, PUE, QRO, SLP, SON, TAM, VER, ZAC).

Aristida purpurea var. parishii (Hitchc.) Allred (BC, SON).

Aristida purpurea var. perplexa Allred \& Valdés-Reyna (CHIH, COAH, NL, SLP, ZAC).

Aristida purpurea Nutt. var. purpurea $(\mathrm{CHIH}, \mathrm{COAH}, \mathrm{DGO}, \mathrm{HGO}, \mathrm{NL}, \mathrm{PUE}, \mathrm{SLP}, \mathrm{SON}, \mathrm{TAM}, \mathrm{VER}$, ZAC).

Aristida purpurea var. wrightii (Nash) Allred (AGS, CHIH, COAH, CDM, DGO, MÉX, NL, OAX, PUE, SLP, SIN, TAM, ZAC).

**Aristida purpusiana Hitchc. (BCS).

Aristida schiedeana Trin. \& Rupr.

Aristida schiedeana var. orcuttiana (Vasey) Allred \& Valdés-Reyna (AGS, BC, CHIH, COAH, DGO, GTO, $\mathrm{MICH}, \mathrm{NL}, \mathrm{SIN}, \mathrm{SON})$.

Aristida schiedeana Trin. \& Rupr. var. schiedeana (AGS, BCS, CHIH, CHIS, COAH, CDM, DGO, GTO, GRO, HGO, JAL, MÉX, MICH, MOR, NAY, NL, OAX, PUE, SLP, TAM, TLX, VER, ZAC).

*Aristida scribneriana Hitchc. (AGS, DGO, GTO, GRO, JAL, MICH, ZAC).

*Aristida spanospicula Allred, Valdés-Reyna \& Sánchez-Ken (CHIH, DGO, SIN, SON).

**Aristida tenuifolia Hitchc. (COL).

Aristida ternipes Cav.

Aristida ternipes var. minor (Vasey) Hitchc. (BC, BCS, CAM, CHIH, CHIS, COAH, COL, CDM, DGO, GTO, GRO, HGO, JAL, MÉX, MICH, MOR, NAY, NL, OAX, PUE, QRO, SLP, SIN, SON, TAM, VER, YUC, ZAC).

Aristida ternipes Cav. var. ternipes (AGS, BC, BCS, CAM, CHIH, CHIS, COAH, COL, CDM, DGO, GTO, GRO, HGO, JAL, MÉX, MICH, MOR, NAY, NL, OAX, PUE, QRO, QROO, SLP, SIN, SON, TAM, VER, YUC, ZAC). 
**Aristida tuitensis Sánchez-Ken \& Dávila (JAL).

*Aristida vaginata Hitchc. (COL, JAL).

Arrhenatherum (1/0/0/1; Pooideae)

+Arrhenatherum elatius (L.) P. Beauv. ex J. Presl. \& C. Presl. (CDM).

Arthraxon (1/0/0/1; Panicoideae)

+Arthraxon hispidus (Thunb.) Makino

+Arthraxon hispidus var. hispidus (CHIS, COL, MICH).

Arthrostylidium (1/0/0/0; Bambusoideae)

Arthrostylidium excelsum Griseb. (CHIS).

Arundinella (3/0/0/0; Panicoideae)

Arundinella berteroniana (Schult.) Hitchc. \& Chase (CHIS, COL, CDM, GRO, HGO, JAL, MÉX, MICH, MOR, NL, OAX, PUE, SLP, SIN, TAB, TAM, VER).

Arundinella deppeana Nees ex Steud. (CAM, CHIS, COL, CDM, GRO, HGO, JAL, MÉX, MICH, MOR, NAY, NL, OAX, PUE, QROO, SIN, TAB, VER, YUC).

Arundinella hispida (Humb. \& Bonpl. ex Willd.) Kuntze (CHIS, CDM, DGO, GRO, JAL, MÉX, MICH, NAY, OAX, SON, TAB, VER).

Arundo (1/0/0/1; Arundinoideae)

+ Arundo donax $\mathrm{L}$.

+Arundo donax L. fo. donax (CHIH, COAH, COL, CDM, DGO, GTO, GRO, HGO, JAL, MÉX, MOR, NAY, NL, OAX, PUE, PUE, QRO, QROO, SIN SON, TAM, VER, YUC, ZAC).

+Arundo donax fo. versicolor (Mill.) Beetle (CHIH, CHIS, COAH, COL, GTO, GRO, HGO, JAL, MÉX, MOR, NAY, NL, OAX, PUE, QRO, SIN, SON, TAM, VER, ZAC).

Aulonemia (1/1/0/0; Bambusoideae)

*Aulonemia laxa (F. Maek.) McClure (CHIS, GRO, VER).

Avena $(2 / 0 / 0 / 2$; Pooideae)

+Avena fatua L. (AGS, BC, CHIH, CHIS, COAH, CDM, DGO, GTO, HGO, JAL, MÉX, MICH, MOR, NL, OAX, PUE, SLP, SON, TAM, TLX, VER, YUC).

+Avena sativa L. (AGS, BC, CHIH, CHIS, COAH, CDM, DGO, HGO, JAL, MÉX, MICH, MOR, NL, OAX, PUE, SON, VER).

Avenella (1/0/0/0; Pooideae)

Avenella flexuosa (L.) Drejer (MÉX).

Axonopus (12/2/1/0; Panicoideae)

Axonopus arsenei Swallen (COL, MÉX, MICH, OAX).

Axonopus aureus P. Beauv. (CHIS, OAX, TAB).

Axonopus centralis Chase (CHIS, COL, GRO, JAL, NAY).

Axonopus chrysoblepharis (Lag.) Chase (CHIS).

Axonopus compressus (Sw.) P. Beauv. (CAM, CHIS, COL, CDM, GRO, HGO, JAL, MÉX, MICH, NAY, NL, OAX, PUE, QRO, QROO, SIN, SON, TAB, TAM, VER, YUC).

*Axonopus deludens Chase (JAL, NAY, SIN).

Axonopus fissifolius (Raddi) Kuhlm. (CAM, CHIS, HGO, JAL, OAX, PUE, QRO, QROO, TAB, VER, YUC).

*Axonopus mexicanus G.A. Black (DGO, SIN).

Axonopus poiophyllus Chase (CHIS, OAX, PUE, TAM, VER).

Axonopus purpusii (Mez) Chase (CAM, CHIS, COL, OAX, PUE, TAB, VER).

**Axonopus rosei (Scribn. \& Merr.) Chase (NAY).

Axonopus scoparius (Flüggé) Kuhlm. (CHIS, OAX).

Bambusa (3/0/0/3; Bambusoideae)

+ Bambusa multiplex (Lour.) Raeusch. ex Schult. \& Schult. f. (CHIS).

+Bambusa tuldoides Munro (CHIS).

+Bambusa vulgaris Schrad. ex J.C. Wendl. (CAM, CHIS, COAH, CDM, JAL., MÉX, MOR, NAY, NL, OAX, SON, TAM, VER, YUC).

Blepharidachne (1/0/0/0; Chloridoideae)

Blepharidachne bigelovii (S. Watson) Hack. (COAH, NL). 
Bothriochloa (16/0/0/1; Panicoideae) Bothriochloa alta (Hitchc.) Henrard (CHIH, CHIS, COAH, DGO, GTO, HGO, JAL, MÉX, MICH, MOR, NAY, OAX, QRO, SLP TAM, ZAC, VER).

Bothriochloa barbinodis (Lag.) Herter (AGS, BC, BCS, CHIH, CHIS, COAH, COL, CDM, DGO, GTO, GRO, HGO, JAL, MÉX, MICH, MOR, NAY, NL, OAX, PUE, QRO, SLP, SON, VER, ZAC).

+Bothriochloa bladhii (Retz.) S.T. Blake (YUC).

Bothriochloa edwardsiana (Gould) Parodi (OAX).

Bothriochloa hirtifolia (J. Presl) Henrard (CHIS, COAH, CDM, GTO, GRO, JAL, MÉX, MICH, MOR, NAY, OAX, PUE, VER).

Bothriochloa hybrida (Gould) Gould (COAH, CDM, MÉX, NL, OAX, OAX, QRO, SLP, TAM).

Bothriochloa ischaemum (L.) Keng

+ Bothriochloa ischaemum (L.) Keng var. ischaemum (CAM, COAH, MICH, NAY, NL, PUE, QROO, SON, TAM, YUC).

+ Bothriochloa ischaemum var. songarica (Rupr. ex Fisch. \& Meyen) Celarier \& J.R. Harlan (CAM, COAH, MICH, NAY, NL, OAX, PUE, QROO, SON, TAM, YUC).

Bothriochloa laguroides (DC.) Herter

Bothriochloa laguroides (DC) Herter var. laguroides (CHIS, CDM, GTO, HGO, MÉX, MOR, OAX, PUE, TAB, TLX, VER).

Bothriochloa laguroides var. torreyana (Steud.) M. Marchi \& Longhi-Wagner (BC, CHIH, COAH, JAL,

$\mathrm{MICH}, \mathrm{NL}, \mathrm{QRO}, \mathrm{SLP}, \mathrm{SON}, \mathrm{TAM}, \mathrm{ZAC}$ ).

Bothriochloa longipaniculata (Gould) Allred \& Gould (CHIH, COAH, NL, TAM).

Bothriochloa palmeri (Hack.) Pilg. (DGO, GTO, JAL, MICH, ZAC).

Bothriochloa perforata (Trin. ex. E. Fourn.) Herter (CHIH, CDM, DGO, GTO, HGO, JAL, MÉX, MICH, OAX, SLP, VER, ZAC).

Bothriochloa pertusa (L.) A. Camus (CAM, CHIS, HGO, NAY, NL, OAX, QRO, QROO, SLP, SON, TAM, VER, YUC).

Bothriochloa reevesii (Gould) Gould (COAH, HGO, MÉX, NL, OAX, PUE, SLP, TAM).

Bothriochloa saccharoides (Sw.) Rydb.

Bothriochloa saccharoides var. parvispicula (Hitchc.) Tovar (CHIS, OAX).

Bothriochloa saccharoides (Sw.) Rydb. var. saccharoides (DGO, QROO, VER).

Bothriochloa springfieldii (Gould) Parodi (CHIH, COAH, DGO, NAY, PUE, ZAC).

Bothriochloa wrightii (Hack.) Henrard (CHIH, DGO, GTO, MOR, NL, SLP, VER, ZAC).

Bouteloua (52/16/6/0; Chloridoideae)

Bouteloua alamosana Vasey (CHIS, OAX, SON).

Bouteloua americana (L.) Scribn. (CAM, CHIS, YUC).

*Bouteloua annua Swallen (BCS, SON).

Bouteloua aristidoides (Kunth) Griseb.

Bouteloua aristidoides (Kunth) Griseb. var. aristidoides (AGS, BC, BCS, CHIH, COAH, COL, DGO, JAL, NAY, NL, OAX, QRO, SLP, SIN, SON, ZAC).

Bouteloua aristidoides var. arizonica M.E. Jones (CHIH, SON).

Bouteloua barbata Lag.

Bouteloua barbata Lag. var. barbata (AGS, BC, BCS, CHIH, COAH, COL, CDM, DGO, GTO, GRO,

HGO, JAL, MÉX, NAY, NL, OAX, PUE, QRO, SLP, SIN, SON, ZAC).

Bouteloua barbata var. rothrockii (Vasey) Gould (BCS, CHIH, DGO, JAL, SLP, SIN, SON).

*Bouteloua barbata var. sonorae (Griffiths) Gould (BC, BCS, SIN, SON).

**Bouteloua bracteata (McVaugh) Columbus (MICH).

Bouteloua breviseta Vasey $(\mathrm{CHIH})$.

*Bouteloua chasei Swallen (COAH, NL, S.L.P, ZAC).

**Bouteloua chihuahuana (M.C. Johnston) Columbus (CHIH).

Bouteloua chondrosioides (Kunth) Benth. ex S. Watson (AGS, CHIH, CHIS, COAH, CDM, DGO, GTO, GRO, JAL, MÉX, MICH, NL, OAX, PUE, SLP, SON, TAM, ZAC).

Bouteloua curtipendula (Michx.) Torr.

Bouteloua curtipendula var. caespitosa Gould \& Kapadia (AGS, BC, BCS, CHIH, CHIS, COAH, CDM, DGO, GTO, GRO, HGO, JAL, MÉX, MICH, MOR, NAY, NL, OAX, PUE, QRO, SLP, SIN, SON, TAM, TLX, VER, ZAC).

Bouteloua curtipendula (Michx.) Torr. var. curtipendula (CHIH, COAH, CDM, NL, PUE, TAM, ZAC). Bouteloua curtipendula var. tenuis Gould \& Kapadia (AGS, CHIH, CHIS, COAH, CDM, DGO, GTO, JAL, MÉX, MICH, MOR, NL, OAX, PUE, QRO, SLP, TAM, TLX, VER, ZAC).

Bouteloua dactyloides (Nutt.) Columbus (AGS, CHIH, COAH, CDM, DGO, GTO, HGO, JAL, MÉX, NL, PUE, QRO, SLP, SON, TAM, TLX, ZAC).

Bouteloua dimorpha Columbus (CAM, CHIS, COL, GRO, JAL, MÉX, MICH, MOR, NAY, OAX, PUE, QRO, S.L.P, VER, YUC).

*Bouteloua distans Swallen (NL, OAX, PUE).

Bouteloua disticha (Kunth) Benth. (CAM, GRO, JAL, MICH, MOR, NAY, NL, OAX, TAM, YUC). 
Bouteloua diversispicula Columbus (AGS, CHIH, CHIS, COL, GTO, GRO, JAL, MICH, MOR, NAY, OAX, PUE, SLP SIN, SON, ZAC).

*Bouteloua elata Reeder \& C. Reeder (CHIS, COL, GRO, JAL, NAY, OAX).

Bouteloua eludens Griffiths ( $\mathrm{CHIH}, \mathrm{COAH}, \mathrm{SON})$.

Bouteloua erecta (Vasey \& Hack.) Columbus (CHIH, COAH, NL, SON, TAM, ZAC).

Bouteloua eriopoda (Torr.) Torr. (CHIH, COAH, SON, TAM, ZAC).

**Bouteloua eriostachya (Swallen) Reeder (COAH).

Bouteloua gracilis (Kunth) Lag. ex Griffiths (AGS, CHIH, COAH, CDM, DGO, GRO, GTO, HGO, JAL, MÉX,

NL, OAX, PUE, QRO, SLP, SON, TAM, TLX, VER, ZAC).

*Bouteloua griffithsii Columbus (GRO, JAL, MICH, MOR, OAX, PUE, QRO).

**Bouteloua herrera-arrietae P.M. Peterson \& Romasch. (NL).

Bouteloua hirsuta Lag.

Bouteloua hirsuta var. glandulosa (Cerv.) Gould (AGS, BCS, CHIH, CHIS, CDM, DGO, GTO, GRO,

HGO, JAL, MÉX, MICH, MOR, NAY, OAX, SON, TLX, VER, ZAC).

Bouteloua hirsuta Lag. var. hirsuta (AGS, BCS, CHIH, CHIS, COAH, CDM, DGO, GTO, HGO, JAL, MÉX, MICH, MOR, NAY, NL, OAX, PUE, SLP, SIN, SON, TAB, TAM, TLX, VER, ZAC).

**Bouteloua johnstonii Swallen (COAH).

*Bouteloua karwinskii (E. Fourn.) Griffiths (COAH, NL, SLP, TAM, ZAC).

Bouteloua media (E. Fourn.) Gould \& Kapadia (BCS, CHIS, COL, CDM, GRO, JAL, MÉX, MICH, MOR, NAY, OAX, PUE, SLP, VER).

Bouteloua mexicana (Scribn.) Columbus (GRO).

*Bouteloua multifida (Griffiths) Columbus (GRO, MÉX, MICH, MOR, OAX, PUE, SON).

*Bouteloua nervata Swallen (HGO, MÉX).

Bouteloua parryi (E. Fourn.) Griffiths

*Bouteloua parryi var. gentryi (Gould) Gould (DGO, JAL, SIN).

Bouteloua parryi (E. Fourn.) Griffiths var. parryi (CHIH, COAH, COL, CDM, DGO, GTO, JAL, MÉX, MOR, NL, PUE, SLP, SIN, SON, ZAC).

*Bouteloua pedicellata Swallen (GTO, HGO, NL, PUE, TLX, VER).

*Bouteloua polymorpha (E. Fourn.) Columbus (AGS, CDM, DGO, GTO, GRO, JAL, MÉX, MICH, MOR, NAY, OAX, PUE, QRO, SLP, SIN, SON, ZAC).

*Bouteloua purpurea Gould \& Kapadia (CDM, GTO, HGO, MÉX, MICH, QRO, SLP).

**Bouteloua quiriegoensis Beetle (SON).

Bouteloua radicosa (E. Fourn.) Griffiths (AGS, BCS, CHIH, COAH, CDM, DGO, GTO, GRO, HGO, JAL, MÉX, MICH, MOR, NAY, NL, OAX, PUE, SLP, SON, TAM, TLX, VER, ZAC).

Bouteloua ramosa Scribn. ex Vasey (CHIH, COAH, DGO, NL, TAM, ZAC).

*Bouteloua reederorum Columbus (OAX, PUE).

*Bouteloua reflexa Swallen (BC, BCS, NAY, SIN, SON). ,

Bouteloua repens (Kunth) Scribn. \& Merr. (AGS, BCS, CAM, CHIH, CHIS, COAH, COL, CDM, DGO, GTO, GRO, HGO, JAL, MÉX, MICH, MOR, NAY, NL, OAX, PUE, QRO, SLP, SIN, SON, TAB, TAM, VER, YUC,

ZAC).

Bouteloua rigidiseta (Steud.) Hitchc. (COAH, DGO, NL, SON, TAM).

Bouteloua scabra (Kunth) Columbus (CHIS, HGO, QRO, TAB).

*Bouteloua scorpioides Lag. (AGS, BC, BCS, CHIH, COAH, CDM, DGO, GTO, GRO, HGO, JAL, MÉX, MOR, NL, OAX, PUE, QRO, SLP, TAM, TLX, ZAC).

Bouteloua simplex Lag. (AGS, BC, BCS, CHIH, COAH, CDM, DGO, GTO, GRO, HGO, JAL, MÉX, MOR, NL, OAX, PUE, QRO, SLP, SON, TAM, TLX, VER, ZAC).

*Bouteloua stolonifera Scribn. (AGS, CDM, DGO, HGO, MÉX, SLP, ZAC).

Bouteloua triaena (Trin. ex Spreng.) Scribn. (CAM, CHIS, COAH, COL, CDM, GTO, GRO, HGO, JAL, MÉX, $\mathrm{MICH}$, MOR, OAX, PUE, QROO, SIN, YUC).

Bouteloua trifida Thurb. (BC, CHIH, COAH, GTO, NL, SLP, SON, TAM).

Bouteloua uniflora Vasey

*Bouteloua uniflora var. coahuilensis Gould \& Kapadia (AGS, COAH, DGO, GTO, NL, QRO, TAM,

ZAC).

Bouteloua uniflora Vasey var. uniflora (AGS, COAH, NL, PUE, QRO, SLP, TAM).

*Bouteloua varia (Swallen) Columbus (JAL, MICH, OAX, PUE).

Bouteloua warnockii Gould \& Kapadia (CHIH, COAH, NL, SON, TAM).

Bouteloua williamsii Swallen (AGS, CHIS, GRO, JAL, MÉX, NAY, OAX, PUE, ZAC).

Brachiaria (1/0/0/1; Panicoideae)

+ Brachiaria eruciformis (Sm.) Griseb. (COAH).

Brachypodium (3/2/0/1; Pooideae)

+Brachypodium distachyon (L.) P. Beauv. (BC).

*Brachypodium mexicanum (Roem. \& Schult.) Link

Brachypodium mexicanum var. inerme Beetle (COAH, NL, TAM). 
Brachypodium mexicanum (Roem. \& Schult.) Link var. mexicanum (BCS, CHIS, COAH, CDM, DGO, GTO, GRO, HGO, JAL, MÉX, MICH, MOR, NL, OAX, PUE, QRO, SLP, TAM, TLX, VER, ZAC). *Brachypodium pringlei Scribn. ex Beal (COAH, NL, TAM).

Briza (1/0/0/1; Pooideae) + Briza minor L. (CHIS, CDM, MÉX, MICH, MOR, OAX, PUE, VER).

Bromidium (1/0/0/0; Pooideae) Bromidium tandilense (Kuntze) Rúgolo (BC).

Bromus (22/2/1/6; Pooideae)

Bromus anomalus Rupr. ex E. Fourn. (AGS, BCS, CHIH, CHIS, COAH, CDM, DGO, GTO, GRO, HGO, JAL, MÉX, MICH, MOR, NL, OAX, PUE, QRO, S.LP., TAM, TLX, VER, ZAC).

Bromus arizonicus (Shear) Stebbins (BC, SON).

*Bromus attenuatus Swallen (HGO, NL, SLP, TAM).

Bromus berteroanus Colla (BC, SON).

Bromus carinatus Hook. \& Arn.

Bromus carinatus Hook. \& Arn. var. carinatus $(\mathrm{BC}, \mathrm{CHIH}, \mathrm{CHIS}, \mathrm{COAH}, \mathrm{CDM}, \mathrm{DGO}, \mathrm{GTO}, \mathrm{HGO}, \mathrm{JAL}$, MÉX, MICH, MOR, OAX, PUE, SLP, TAM, TLX, VER).

Bromus carinatus var. marginatus (Nees) Barkworth \& Anderton (AGS, BC, CHIH, CHIS, COAH, CDM, DGO, GTO, GRO, HGO, JAL, MÉX, MICH, MOR, NAY, NL, OAX, PUE, QRO, SLP, SIN, SON, TAM, TLX, VER, ZAC).

+ Bromus catharticus Vahl

+ Bromus catharticus Vahl. var. catharticus (AGS, BC, CHIH, COAH, CDM, DGO, HGO, JAL, NL, PUE, QRO, SLP, SON, TLX, VER, ZAC).

*Bromus densus Swallen (COAH, NL, SLP, TAM).

+Bromus diandrus Roth (BC, COL, CDM, MÉX, MOR, OAX, PUE, SLP, TLX, VER).

Bromus dolichocarpus Wagnon (CHIS, CDM, DGO, GRO, HGO, JAL, MÉX, MICH, MOR, OAX, PUE, VER). Bromus exaltatus Bernh. (CHIS, CDM, GRO, HGO, JAL, MÉX, OAX, PUE, QRO, SLP, SON, TLX, VER).

Bromus frondosus (Shear) Wooton \& Standl. (CHIH, COAH, DGO, SIN, SON).

Bromus hordeaceus L. (BC, BCS).

+ Bromus inermis Leysser (COAH).

Bromus japonicus Houtt. (CHIH, COAH, JAL).

Bromus lanatipes (Shear) Rydb. (COAH).

+Bromus madritensis L. (BC).

**Bromus pinetorum Swallen $(\mathrm{COAH})$.

Bromus pseudolaevipes Wagnon (BC).

Bromus richardsonii Link (BC, BCS, CHIH, COAH, DGO, MICH, NL, QRO, TLX).

+ Bromus rubens L. (BC, BCS, SON).

Bromus secalinus L. (VER).

+Bromus tectorum L. (BC, $\mathrm{CHIH}, \mathrm{COAH})$.

Calamagrostis (12/5/0/0); Pooideae

${ }^{*}$ Calamagrostis erectifolia Hitchc. (COL, JAL, PUE).

${ }^{*}$ Calamagrostis eriantha (Kunth) Steud. (CDM, MÉX. OAX, PUE, VER).

Calamagrostis guatemalensis Hitchc. (CHIS).

Calamagrostis intermedia (J. Presl) Steud. (CDM, MÉX, OAX, VER).

${ }^{*}$ Calamagrostis orizabae (Rupr. ex E. Fourn.) Beal (COL, CDM, MÉX, MOR, OAX, PUE, VER).

*Calamagrostis pringlei Scribn. ex Beal (AGS, CHIH, COAH, DGO, HGO, MICH, NL, ZAC).

Calamagrostis purpurascens $\mathrm{R}$. Br.

Calamagrostis purpurascens $\mathrm{R}$. Br. var. purpurascens (COAH, NL).

Calamagrostis rigescens (J. Presl) Scribn. (VER).

Calamagrostis tolucensis (Kunth) Trin. ex Steud. (CHIS, CDM, GRO, JAL, MÉX, MICH, OAX, PUE, TLX, VER).

*Calamagrostis valida Sohns (COL, DGO, JAL, MICH, SIN).

Calamagrostis viridiflavescens (Poir.) Steud.

Calamagrostis viridiflavescens var. montevidensis (Nees) Kämpf (CHIS).

Calamagrostis vulcanica Swallen (CHIS).

Catapodium (1/0/0/1; Pooideae)

+ Catapodium rigidum (L.) Dony (NL).

Cenchrus (20/4/0/5; Panicoideae)

Cenchrus americanus (L.) Morrone (CHIH, MÉX, NL, OAX).

Cenchrus bambusiformis (E. Fourn.) Morrone (CHIS, CDM, GTO, HGO, JAL, MICH, NL, OAX, PUE, SLP, VER, ZAC). 
+Cenchrus brownii Roem. \& Schult. (BC, CAM, CHIH, CHIS, COAH, COL, CDM, DGO, GRO, JAL, MÉX, MICH, MOR, NAY, NL, OAX, PUE, QRO, QROO, SLP, SIN, TAB, TAM, VER, YUC, ZAC).

+Cenchrus ciliaris L. (AGS, BC, BCS, CAM, CHIH, CHIS, COAH, COL, CDM, DGO, GTO, GRO, HGO, JAL, MICH, NAY, NL, OAX, QROO, SLP, SIN, SON, TAM, VER, ZAC).

+Cenchrus clandestinus (Hochst. ex Chiov.) Morrone (CHIS, CHIH, COL, CDM, DGO, HGO, JAL, MÉX, $\mathrm{MICH}, \mathrm{MOR}, \mathrm{NL}, \mathrm{PUE}, \mathrm{QRO}, \mathrm{SLP}, \mathrm{TLX}, \mathrm{VER}, \mathrm{ZAC})$.

Cenchrus complanatus (Ness) Morrone (VER).

Cenchrus crinitus (Kunth) Morrone (MICH, VER).

${ }^{*}$ Cenchrus distachyus (E. Fourn.) Morrone (CHIS, MÉX, MICH, OAX, PUE, VER).

*Cenchrus durus (Beal) Morrone (CHIH, MÉX, OAX, VER).

Cenchrus echinatus L. (AGS, BCS, CAM, CHIH, CHIS, COAH, COL, CDM, DGO, GTO, GRO, HGO, JAL, MÉX, MICH, MOR, NAY, NL, OAX, PUE, QRO, QROO, SLP, SIN, SON, TAB, TAM, VER, YUC, ZAC).

Cenchrus incertus M.A. Curtis (AGS, BC, BCS, CAM, CHIH, CHIS, COAH, COL, CDM, DGO, GTO, GRO, HGO, JAL, MÉX, MOR, NAY, NL, OAX, PUE, QRO, QROO, S.L.P, SIN, SON, TAB, TAM, TLX, VER, YUC, $\mathrm{ZAC})$.

Cenchrus longispinus (Hack.) Fernald (CHIH, CHIS, DGO, GRO, JAL, MICH, OAX, SON).

Cenchrus multiflorus J. Presl (CHIH, CHIS, COAH, GRO, JAL, MÉX, MICH, NAY, OAX, SON, ZAC).

Cenchrus myosuroides Kunth

Cenchrus myosuroides Kunth var. myosuroides (BC, BCS, SIN, SON).

+Cenchrus orientalis (Rich.) Morrone (COAH, GTO, GRO, MÉX, MICH, MOR, VER).

*Cenchrus palmeri Vasey (BC, BCS, SIN, SON).

Cenchrus pilosus Kunth (CAM, CHIS, COL, CDM, GRO, JAL, MÉX, MICH, MOR, NAY, OAX, PUE, QROO, VER, YUC, ZAC).

Cenchrus polystachios (L.) Morrone (CHIH, CHIS, NAY, OAX, PUE, SIN, VER).

${ }^{*}$ Cenchrus prolificus (Chase), Morrone (GRO, OAX, VER).

+Cenchrus purpureus (Schumach.) Morrone (CAM, CHIS, HGO, JAL, MÉX, MOR, NL, OAX, PUE, QRO, QROO, TAB, VER, YUC).

Chaetium (1/0/0/0; Panicoideae)

Chaetium bromoides (J. Presl) Benth. ex Hemsl. (CAM, CHIH, CHIS, COL, CDM, GRO, JAL, MÉX, MICH, NAY, OAX, PUE, VER).

Chascolytrum (1/0/0/0; Pooideae)

Chascolytrum subaristatum (Lam.) Desv. (CHIS, COAH, COL, CDM, DGO, MÉX, OAX, PUE, SLP, VER, ZAC).

Chasmanthium (2/0/1/0; Panicoideae)

${ }^{* *}$ Chasmanthium curvifolium (Valdés-Reyna, Morden \& S.L. Hatch) Wipff \& S.D. Jones (TAM).

Chasmanthium latifolium (Michx.) H.O. Yates (NL).

Chloris (10/0/1/2; Chloridoideae)

Chloris andropogonoides E. Fourn. (COAH, NL, SLP, TAM).

Chloris barbata Sw. (BCS, CAM, CHIS, COL, GRO, JAL, MICH, OAX, QROO, SLP, SIN, TAB, TAM, VER, YUC).

Chloris cucullata Bisch. (COAH, NL, TAM, YUC).

+Chloris gayana Kunth (AGS, CHIH, CHIS, COAH, COL, DGO, GTO, JAL, MÉX, MICH, MOR, NL, OAX, QRO, SLP, TAB, TAM, VER, YUC, ZAC).

Chloris pycnothrix Trin. (GTO, GRO, JAL, MICH, MOR, PUE).

Chloris radiata (L.) Sw. (GTO, GRO, JAL, MICH, OAX, SLP, TAB, VER).

Chloris rufescens Lag. (AGS, CHIS, COAH, COL, CDM, DGO, GTO, GRO, HGO, JAL, MÉX, MICH, MOR, NL, OAX, PUE, QRO, SLP, VER, ZAC).

Chloris submutica Kunth (AGS, CHIH, CHIS, COAH, CDM, DGO, GTO, HGO, JAL, MÉX, MICH, NAY, NL, OAX, PUE, QRO, SLP, SON, TAM, TLX, VER, ZAC).

${ }^{* *}$ Chloris truncata R. Br. (SON).

+Chloris virgata Sw. (AGS, BC, BCS, CAM, CHIH, CHIS, COAH, COL, CDM, DGO, GTO, GRO, HGO, JAL, MÉX, MICH, MOR, NAY, OAX, PUE, QRO, QROO, SLP, SIN, SON, TAB, TAM, VER, YUC, ZAC).

Chrysopogon (1/0/0/1; Panicoideae)

+Chrysopogon zizanioides (L.) Roberty (CHIS, VER).

Chusquea (21/8/5/0; Bambusoideae)

${ }^{*}$ Chusquea aperta L.G. Clark (OAX, VER).

*Chusquea bilimekii E. Fourn. (MÉX, VER).

${ }^{*}$ Chusquea circinata Soderstr. \& C.E. Calderón (CHIS, COL, JAL, MICH, NAY, OAX).

Chusquea coronalis Soderstr. \& C.E. Calderón (CHIS).

Chusquea cortesii L.G. Clark \& Ruíz-Sánchez (CHIS, GRO, JAL, MÉX, MICH, NAY). 
**Chusquea enigmatica Ruíz-Sánchez, L.G. Clark \&Mejia-Saulés (VER). ${ }^{*}$ Chusquea galeottiana Rupr. ex Munro (GRO, OAX).

*Chusquea gibcooperi Ruíz-Sánchez, Mejia-Saulés, Cortes \& L.G. Clark (HGO, PUE, VER).

**Chusquea glauca L.G. Clark (VER).

Chusquea lanceolata Hitchc. (CHIS).

Chusquea liebmannii E. Fourn. (CHIS, COL, GRO, JAL, MICH, OAX, SIN).

Chusquea longifolia Swallen (CHIS).

**Chusquea matlatzinca L.G. Clark \& Ruíz-Sánchez (MICH).

${ }^{* *}$ Chusquea mulleri Munro (VER).

**Chusquea nedjaquithii Ruíz-Sánchez, Mejia-Saulés \& L.G. Clark (OAX).

*Chusquea nelsonii Scribn. \& J.G. Sm. (GRO, MICH).

*Chusquea perotensis L.G. Clark, G. Cortés \& Cházaro (OAX, VER).

Chusquea pittieri Hack. (CHIS).

${ }^{*}$ Chusquea repens L.G. Clark \& Londoño, Nordic J. Bot. 11(3): 327.1991.

${ }^{* *}$ Chusquea repens subsp. oaxacensis L.G. Clark \& Londoño (OAX).

${ }^{*}$ Chusquea repens L.G. Clark \& Londoño subsp. repens (CHIS, OAX).

Chusquea simpliciflora Munro (CHIS).

Chusquea sulcata Swallen (CHIS, OAX, VER).

Cinna (1/0/0/0; Pooideae)

Cinna poiformis (Kunth) Scribn. \& Merr. (CHIS, CDM, HGO, MÉX, MICH, MOR, OAX, PUE, VER).

Coix (1/0/0/1; Panicoideae)

+ Coix lacryma-jobi L.

+Coix lacryma-jobi L. var. lacryma-jobi (CHIS, COAH, CDM, JAL, MÉX, NL, OAX, TAB, VER, YUC).

Coleataenia (3/0/0/0; Panicoideae)

Coleataenia caricoides (Nees ex Trin.) Soreng (CHIS).

Coleataenia stenodes (Griseb.) Soreng (CHIS).

Coleataenia tenera (Beyr. ex Trin.) Soreng (QROO).

Cortaderia (1/0/0/1; Danthonioideae)

+Cortaderia selloana (Schult. \& Schult.f.) Asch. \& Graebn. (BC, CHIH, CHIS, COAH, CDM, DGO, GTO, MÉX, $\mathrm{MICH}, \mathrm{MOR}, \mathrm{NL}, \mathrm{PUE}, \mathrm{QRO}, \mathrm{SON}, \mathrm{VER})$.

Cottea (1/0/0/0; Chloridoideae)

Cottea pappophoroides Kunth (CHIH, COAH, DGO, JAL, NL, OAX, PUE, SLP, SON, ZAC).

Cryptochloa (1/0/0/0; Bambusoideae)

Cryptochloa strictiflora (E. Fourn.) Swallen (CHIS, OAX, PUE, TAB, VER).

Ctenium (2/2/0/0; Chloridoideae)

*Ctenium planifolium (J. Presl) Kunth (CHIS, MÉX, OAX, ZAC).

${ }^{*}$ Ctenium plumosum (Hitchc.) Swallen (JAL, NAY, SIN).

Cymbopogn (2/0/0/2; Panicoideae)

+Cymbopogon citratus (DC.) Stapf (CAM, CHIS, JAL, NL, OAX, TAB, VER, YUC).

+Cymbopogon nardus (L.) Rendle (COAH, NL, OAX, YUC).

Cynodon (3/0/0/3; Chloridoideae)

+Cynodon dactylon (L.) Pers. (AGS, BC, BCS, CAM, CHIH, CHIS, COAH, COL, CDM, DGO, GTO, GRO, HGO, JAL, MÉX, MICH, MOR, NAY, NL, OAX, PUE, QRO, QROO, SLP, SIN, SON, TAB, TAM, TLX, VER, YUC, ZAC).

+Cynodon nlemfuensis Vanderyst (AGS, CAM, CHIS, JAL, OAX, QROO, TAB, TAM, VER, YUC). +Cynodon plectostachyus (K. Schum.) Pilg. (BC, CAM, COL, DGO, HGO, NAY, NL, OAX, PUE, QRO, QROO, SLP, SON, TAB, TAM, VER, YUC).

Cynosurus (1/0/0/1; Pooideae)

+Cynosurus echinatus L. (BC).

Cyrtococcum (1/0/0/1; Panicoideae)

+Cyrtococcum trigonum (Retz.) A. Camus (CHIS).

Dactylis (1/0/0/1; Pooideae)

+Dactylis glomerata L. (BC, CHIH, CHIS, COAH, CDM, MÉX, MICH, NL, OAX, PUE, SLP, VER). 
Dactyloctenium (4/0/0/4; Chloridoideae)

+ Dactyloctenium aegyptium (L.) Willd. (AGS, BC, BCS, CAM, CHIH, CHIS, COAH, COL, CDM, DGO, GTO, GRO, JAL, MÉX, MICH, MOR, NAY, NL, OAX, PUE, QRO, QROO, SLP, SIN, SON, TAB, TAM, VER, YUC,

ZAC).

+ Dactyloctenium aristatum Link (YUC).

+Dactyloctenium geminatum Hack. (CAM, GRO, QROO).

+ Dactyloctenium scindicum Boiss. (BCS, YUC).

Danthonia (3/0/1/0; Danthonioideae)

**Danthonia chiapasensis Davidse (CHIS).

Danthonia secundiflora J. Presl

Danthonia secundiflora J. Presl subsp. secundiflora (CHIS, HGO, PUE).

Danthonia spicata (L.) P. Beauv. ex Roem. \& Schult. (COAH, HGO, PUE, TAM, VER).

Dasyochloa (1/0/0/0; Chloridoideae)

Dasyochloa pulchella (Kunth) Willd. ex Rydb. (AGS, BC, CHIH, COAH, CDM, DGO, GTO, HGO, JAL, MÉX, NL, QRO, SLP, SON, TAM, ZAC).

+Dendrocalamopsis (1/0/0/1; Bambusoideae)

+Dendrocalamopsis oldhamii (Munro) Keng f. (CHIS, VER).

Deschampsia (4/1/0/0; Pooideae)

Deschampsia cespitosa (L.) P. Beauv. (BC, COAH).

Deschampsia danthonioides (Trin.) Munro (BC, $\mathrm{CHIH})$.

Deschampsia elongata (Hook.) Munro (BC, CHIH, CDM, HGO, JAL, MÉX, MICH, MOR, VER).

*Deschampsia liebmanniana (E. Fourn.) Hitchc. (CDM, JAL, MÉX, MICH, PUE, VER).

Dichanthelium (16/1/0/0; Panicoideae)

Dichanthelium aciculare (Desv. ex Poir.) Gould \& C.A. Clark

Dichanthelium aciculare (Desv. ex Poir.) Gould \& C.A. Clark var. aciculare (CHIS, NL, OAX, TAM, VER).

Dichanthelium aciculare var. ramosum (Griseb.) Davidse (CAM, CHIS, TAB).

Dichanthelium acuminatum (Sw.) Gould \& C.A. Clark

Dichanthelium acuminatum (Sw.) Gould \& C.A. Clark var. acuminatum (CAM, CHIS, COAH, GRO, NL, OAX, PUE, SLP, TAB, TAM, VER).

Dichanthelium acuminatum var. lindheimeri (Nash) Gould \& C.A. Clark (COAH, NL, PUE, TAM, VER). Dichanthelium acuminatum var. longiligulatum (Nash) Gould \& C.A. Clark (CHIS, TAB).

Dichanthelium commutatum (Schult.) Gould (CHIS, HGO, JAL, MÉX, MICH, MOR, OAX, PUE, QRO, TAM, VER).

Dichanthelium dichotomum (L.) Gould

Dichanthelium dichotomum (L.) Gould var. dichotomum (CHIS, GRO, HGO, OAX, PUE, QROO, SLP, VER).

Dichanthelium ensifolium (Baldwin ex Elliott) Gould

Dichanthelium ensifolium (Baldwin ex Elliott) Gould var. ensifolium (CHIS, HGO, VER).

Dichanthelium laxiflorum (Lam.) Gould (CHIS, HGO, MÉX, NL, OAX, PUE, QRO, SLP, TAM, VER).

*Dichanthelium macrospermum Gould (QRO, SLP).

Dichanthelium nodatum (Hitchc. \& Chase) Gould (COAH, TAM).

Dichanthelium oligosanthes (Schult.) Gould (CHIH, COAH, NL, SON, TAM, VER).

Dichanthelium ovale (Elliott) Gould \& C.A. Clark (CHIS, MÉX, PUE, SLP, TAM, VER).

Dichanthelium pedicellatum (Vasey) Gould (COAH, HGO, NL, OAX, TAM).

Dichanthelium portoricense (Desv. ex Ham.) B.F. Hansen \& Wunderlin (VER).

Dichanthelium sphaerocarpon (Elliott) Gould

Dichanthelium sphaerocarpon (Elliott) Gould var. sphaerocarpon $(\mathrm{CHIH}, \mathrm{CHIS}, \mathrm{DGO}, \mathrm{GRO}, \mathrm{JAL}, \mathrm{MICH}$, PUE, QROO, VER, YUC, ZAC).

Dichanthelium strigosum (Muhl. ex Elliot) Freckman

Dichanthelium strigosum (Muhl. ex Elliot) Freckman var. strigosum (CAM, CHIS, OAX, VER).

Dichanthelium umbonulatum (Swallen) Davidse (OAX).

Dichanthelium viscidellum (Scribn.) Gould (CAMS, CHIS, HGO, MÉX, OAX, PUE, QROO, VER).

Dichanthium (3/0/0/3; Panicoideae)

+Dichanthium annulatum (Forssk.) Stapf (AGS, CAM, CHIH, COAH, JAL, MOR, NL, OAX, QROO, SLP, TAM, YUC).

+Dichanthium aristatum (Poir.) C.E. Hubb. (CAM, NAY, NL, QROO, SLP, TAM).

+ Dichanthium sericeum (R. Br.) A. Camus (CAM, SON, TAM). 
Digitaria (31/4/1/6; Panicoideae)

+Digitaria abyssinica (Hochst. ex A. Rich.) Stapf (VER).

Digitaria arenicola (Swallen) Beetle (TAM).

Digitaria argillacea (Hitchc. \& Chase) Fernald (CHIS, COL, DGO, GTO, JAL, MÉX, MICH, MOR, NAY, OAX, SON).

Digitaria badia (Scribn. \& Merr.) Fernald (CHIS, CDM, GTO, HGO, JAL, MÉX, MICH, OAX, QRO, TLX, ZAC). Digitaria bakeri (Nash) Fernald (CHIS, VER).

+Digitaria bicornis (Lam.) Roem. \& Schult. (BCS, CAM, CHIH, CHIS, COL, CDM, GRO, HGO, JAL, MÉX, MOR, NAY, NL, OAX, PUE, QROO, SIN, SON, TAB, TAM, VER, YUC).

*Digitaria breedlovei R.W. Pohl \& Davidse (CHIS, DGO).

Digitaria californica (Benth.) Henrard (AGS, BC, BCS, CHIH, COAH, DGO, GTO, HGO, JAL, NL, PUE, QRO, SLP, SIN, SON, TAM, ZAC).

Digitaria ciliaris (Retz.) Koeler (AGS, BC, BCS, CAM, CHIH, CHIS, COAH, COL, CDM, DGO, GTO, GRO, HGO, JAL, MÉX, MICH, MOR, NAY, NL, OAX, PUE, QRO, QROO, SLP SIN, SON, TAB, TAM, VER, YUC). Digitaria curtigluma Hitchc. (CHIS, CDM, GTO, JAL, MÉX, MICH, OAX, PUE).

+Digitaria eriantha Steud. (OAX, TAM, VER, ZAC).

Digitaria filiformis (L.) Koeler

Digitaria filiformis (L.) Koeler var. filiformis (CHIH, CHIS, COAH, CDM, DGO, GTO, JAL, MÉX, MICH,

NAY, OAX, SON, VER, ZAC).

Digitaria filiformis var. laeviglumis (OAX).

Digitaria hitchcockii (Chase) Stuck. (COAH, NL, OAX, QRO, SLP, TAM).

Digitaria horizontalis Willd. (CHIS, COAH, COL, GRO, JAL, MÉX, MICH, MOR, NAY, NL, OAX, PUE, QRO, QROO, SLP, SIN, SON, TAB, TAM, VER, YUC).

Digitaria insularis (L.) Mez ex Ekman (AGS, CAM, CHIH, CHIS, COAH, COL, DGO, GTO, GRO, HGO, JAL, MOR, NAY, NL, OAX, PUE, QRO, QROO, SLP, SON, TAB, TAM, VER, YUC).

Digitaria leucites (Trin.) Henrard (CHIS, CDM, DGO, HGO, JAL, MÉX, MICH, MOR, NL, OAX, PUE, SLP, TAB, TLX, VER).

Digitaria leucocoma (Nash) Urb. (CHIS, MÉX, VER).

* Digitaria michoacanensis Sánchez-Ken (MÉX, MICH).

+Digitaria milanjiana (Rendle) Stapf (COL, HGO, JAL, MOR, PUE, QRO, TAB, TAM, VER).

Digitaria nuda Schumach. (CHIS, COL, GRO, GTO, JAL, MÉX, MICH, MOR, NAY, OAX, PUE, QROO, SLP,

SIN, VER, YUC).

Digitaria obtusa Swallen (CHIS, VER).

**Digitaria paniculata Soderstr. ex McVaugh (JAL).

Digitaria patens (Swallen) Henrard (COAH, NL, SON).

Digitaria pubiflora (Vasey) Wipff (CHIH, COAH, DGO, GTO, JAL, NL, SLP, SON, TAM, VER, ZAC).

+Digitaria sanguinalis (L.) Scop. (AGS, BCS, CHIH, DGO, JAL, MICH, MOR, NL, QRO, SLP, SON, TAB, TAM, VER, ZAC).

Digitaria sellowii (Müll. Hal.) Henrard (CHIS, HGO, NL, OAX, PUE, QRO, QROO, SLP, TAB, TAM, VER, YUC).

*Digitaria setigera Roth, in Roem. \& Schult. (CAM, CHIS, OAX, VER).

*Digitaria ternata (A. Rich.) Stapf (AGS, CDM, DGO, GTO, HGO, JAL, MÉX, MICH, MOR, OAX, PUE, QRO, ZAC).

Digitaria texana Hitchc. (VER).

+Digitaria velutina (Forssk.) P. Beauv. (CDM, MOR, OAX, PUE).

Digitaria violascens Link (CHIS, CDM, NL, VER).

Dinebra (6/1/0/0; Chloridoideae)

* Dinebra aquatica (Scribn. \& Merr.) P.M. Peterson \& N. Snow (GRO, JAL, MICH, MOR, NAY, SIN, ZAC).

Dinebra nealleyi (Vasey) P.M. Peterson \& N. Snow (CAM, TAB, TAM, VER, YUC).

Dinebra panicea (Retz.) P.M. Peterson \& N. Snow

Dinebra panicea subsp. brachiata (Steud.) P.M. Peterson \& N. Snow (BC, CAM, CHIH, COAH, DGO,

MICH, MOR, NL, PUE, QROO, SON, TAB, TAM, VER, ZAC).

* Dinebra panicea subsp. mucronata (Steud.) P.M. Peterson \& N. Snow (BC, CHIH, CHIS, COAH, DGO, $\mathrm{MICH}$, MOR, NAY, PUE, QRO, SIN, SON, TAB, TAM, VER, YUC).

Dinebra panicoides (J. Presl) P.M. Peterson \& N. Snow (BC, BCS, CHIH, COL, GRO, JAL, MICH, NAY OAX, SIN, SON, TAB).

Dinebra scabra (Nees) P.M. Peterson \& N. Snow (CHIH, CHIS, MICH, OAX, QROO, SIN, SON, TAB, VER, YUC).

Dinebra viscida (Scribn.) P.M. Peterson \& N. Snow (BC, BCS, CHIH, SIN, SON).

Diplachne (1/0/0/0; Chloridoideae)

Diplachne fusca (L.) P. Beauv ex Roem. \& Schult.

Diplachne fusca subsp. fascicularis (Lam.) P.M. Peterson \& N. Snow (CAM, CHIH, COAH, CDM, DGO,

GTO, HGO, MÉX, MICH, MOR, NL, QRO, QROO, SLP, SIN, SON, YUC, ZAC). 
Diplachne fusca subsp. uninervia (J. Presl) P.M. Peterson \& N. Snow (BCS, CHIH, CHIS, DGO, GTO, GRO, JAL, NAY, OAX, PUE, SLP, SIN, SON, TAM, VER, ZAC).

Disakisperma (1/0/0/0; Chloridoideae)

Disakisperma dubium (Kunth) P.M. Peterson \& N. Snow (AGS, BC, BCS, CHIH, CHIS, COAH, CDM, DGO, GTO, HGO, JAL, MÉX, MICH, MOR, NL, OAX, PUE, QRO, SLP, SIN, SON, TAM, TLX, VER, ZAC).

Distichlis (5/2/1/0; Chloridoideae)

**Distichlis bajaensis H.L. Bell (BC).

${ }^{*}$ Distichlis eludens (Soderstr. \& H.F. Decker) H.L. Bell \& Columbus (DGO, SLP, ZAC).

Distichlis littoralis (Engelm.) H.L. Bell \& Columbus (BC, BCS, CAM, CHIH, CHIS, COAH, MICH, NAY, NL, OAX, SIN, SON, TAM, YUC).

*Distichlis palmeri (Vasey) Fassett (BC, SON).

Distichlis spicata (L.) Greene

*Distichlis spicata var. mexicana Beetle (CHIH, COAH, CDM, DGO, GTO, HGO, JAL, MÉX, MICH, MOR, PUE, SLP, TLX).

Distichlis spicata (L.) Greene var. spicata (CAM, CHIH, CHIS, COAH, CDM, DGO, GTO, HGO, JAL, MÉX, MICH, MOR, OAX, PUE, QROO, SLP, SIN, TAB, TAM, TLX, VER, YUC).

Distichlis spicata subsp. stricta (Torr.) Thorne (DGO).

Echinochloa (10/1/0/0; Panicoideae)

Echinochloa colona (L.) Link (BC, BCS, CAM, CHIH, CHIS, DGO, MÉX, OAX, QROO, TAB, VER, YUC, ZAC). Echinochloa crus-galli (L.) P. Beauv.

Echinochloa crus-galli (L.) P. Beauv. var. crus-galli (AGS, BC, BCS, CHIH, COAH, COL, DGO, GTO, GRO, HGO, JAL, MÉX, MICH, MOR, NAY, NL, OAX, PUE, SLP, SIN, SON, TAB, TAM, VER, ZAC). Echinochloa crus-galli var. mitis (Pursh) Peterm. (CDM, MÉX, MOR, SON).

Echinochloa crus-galli var. zelayensis (Kunth) Hitchc. (AGS, BC, CHIH, CDM, DGO, GTO, HGO, JAL, MÉX, MICH, MOR, NAY, PUE, SON, TAM, ZAC).

Echinochloa crus-pavonis (Kunth) Schult.

Echinochloa crus-pavonis (Kunth) Schult. var. crus-pavonis (BCS, CHIH, CHIS, COAH, DGO, GTO, GRO, HGO, JAL, MÉX, MICH, MOR, NAY, OAX, PUE, QRO, SLP, SIN TAB, VER).

Echinochloa crus-pavonis var. macera (Wiegand) Gould (TAM).

Echinochloa holciformis (Kunth) Chase (AGS, CHIH, CHIS, COAH, CDM, DGO, GTO, HGO, JAL, MÉX, $\mathrm{MICH}, \mathrm{MOR}, \mathrm{NAY}, \mathrm{NL}, \mathrm{PUE}, \mathrm{SLP}, \mathrm{VER}, \mathrm{ZAC})$.

*Echinochloa jaliscana McVaugh (AGS, GTO, JAL, MÉX, MICH, MOR, ZAC).

Echinochloa muricata (P. Beauv.) Fernald

Echinochloa muricata var. microstachya Wiegand (CHIH, DGO, SON).

Echinochloa oplismenoides (E. Fourn.) Hitchc. (AGS, CHIH, CDM, DGO, GRO, HGO, JAL, MÉX, MICH,

OAX, PUE, SON, TAB, TLX, ZAC).

Echinochloa polystachya (Kunth) Hitchc.

Echinochloa polystachya (Kunth) Hitchc. var. polystachya (AGS, CAM, CHIH, CHIS, CDM, DGO, JAL, MICH, OAX, PUE, SON, TAB, TAM, VER, YUC, ZAC).

Echinochloa pyramidalis (Lam.) Hitchc. \& Chase (CAM, CHIS, GRO, NAY, OAX, PUE, TAB, VER, YUC).

Echinochloa walteri (Pursh) A. Heller (CAM, CHIH, COAH, MICH, SLP, TAB, VER, YUC).

Echinolaena (2/0/0/0; Panicoideae)

Echinolaena gracilis Swallen (CHIS, TAB).

Echinolaena standleyi (Hitchc.) Stieber (CHIS).

Eleusine (3/0/0/3; Chloridoideae)

+Eleusine indica (L.) Gaertn. (AGS, BC, BCS, CAM, CHIH, CHIS, COAH, COL, CDM, DGO, GTO, GRO, HGO, JAL, MÉX, MICH, MOR, NAY, NL, OAX, PUE, QRO, QROO, SLP, SIN, SON, TAB, TAM, TLX, VER, YUC, ZAC).

+Eleusine tristachya (Lam.) Lam. (MÉX).

Elionurus (3/0/0/0; Panicoideae)

Elionurus barbiculmis Hack. (AGS, BC, BCS, CHIH, CHIS, COAH, CDM, DGO, GTO, GRO, JAL, MÉX, NAY, PUE, SLP, SIN, SON, TLX, ZAC).

Elionurus ciliaris Kunth (OAX).

Elionurus muticus (Spreng.) Kuntze (JAL).

Elymus (9/2/0/0; Pooideae)

Elymus arizonicus (Scribn. \& J.G. Sm.) Gould (CHIH, DGO, ZAC).

Elymus canadensis L.

Elymus canadensis L. var. canadensis (CHIH, DGO). 
Elymus elymoides (Raf.) Swezey

Elymus elymoides subsp. brevifolius (J.G. Sm.) Barkworth (CHIH, DGO, ZAC).

Elymus glaucus Buckley (BC).

*Elymus pilosus (J. Presl) Á. Löve (GRO, MICH).

*Elymus pringlei Scribn. \& Merr. (AGS, COAH, HGO, MÉX, MICH, NL, PUE, QRO, SLP, VER).

Elymus repens (L.) Gould

+Elymus repens (L.) Gould subsp. repens (CHIH, DGO, ZAC).

Elymus riparius Wiegand (DGO).

Elymus trachycaulus (Link) Gould ex Shinners

Elymus trachycaulus subsp. subsecundus (Link) Á. Löve \& D. Löve (BC).

Elymus trachycaulus (Link) Gould ex Shinners subsp. trachycaulus (CHIH, DGO).

Enneapogon (1/0/0/1; Chloridoideae)

+Enneapogon desvauxii P. Beauv. (AGS, BC, BCS, CHIH, COAH, CDM, DGO, GTO, HGO, JAL, MÉX, NL, OAX, PUE, QRO, SLP, SON, ZAC).

Eragrostis (45/3/0/12; Chloridoideae)

Eragrostis acutiflora (Kunth) Nees (CHIS, COL, GRO, JAL, MICH, NAY, OAX, TAB).

Eragrostis bahiensis Schrad. ex Schult. (CHIS, GTO, JAL, NAY, OAX, TAB, VER).

+Eragrostis barrelieri Daveau (CHIH, CHIS, COAH, DGO, HGO, NL, OAX, PUE, QRO, SLP, TAB, TAM, ZAC). Eragrostis capillaris (L.) Nees (TAM).

+Eragrostis cilianensis (All.) Vignolo ex Janch. (AGS, BC, BCS, CAM., CHIH, CHIS, COAH, DGO, GTO, GRO, HGO, JAL, MÉX, MOR, NL, OAX, PUE, SLP, SIN, SON, TAM, VER, ZAC).

+ Eragrostis ciliaris (L.) R.Br.

+Eragrostis ciliaris (L.) R.Br. var. ciliaris (AGS, BCS, CAM, CHIH, CHIS, COL, CDM, DGO, GRO, JAL,

MÉX, MICH, MOR, NAY, OAX, PUE, QRO, QROO, SLP, SIN, SON, TAB, TAM, VER, YUC).

+Eragrostis ciliaris var. laxa Kuntze (CAMS, QROO, TAM, YUC).

Eragrostis contrerasii R.W. Pohl (CAM, QROO, TAB, VER, YUC).

Eragrostis curtipedicellata Buckley $(\mathrm{COAH}, \mathrm{NL})$.

+Eragrostis curvula (Schrad.) Nees (CHIH, COAH, CDM, DGO, GTO, MICH, NL, QRO, SON, TLX, ZAC).

+ Eragrostis echinochloidea Stapf. (CHIH, SLP).

Eragrostis elliottii S. Watson (CAM, CHIS, JAL, MICH, NL, OAX, QROO, SIN, TAB, TAM, VER, YUC).

Eragrostis erosa Scribn. ex Beal (CHIH, COAH, DGO, NL, SON, TAM, ZAC).

Eragrostis glandulosa L.H. Harv. (CHIH, CHIS, CDM, DGO, GRO, JAL, MÉX, MOR, OAX, ZAC).

Eragrostis guatemalensis Witherspoon (CHIS, MÉX, PUE).

Eragrostis hirsuta (Michx.) Nees (CHIS, NL, OAX, TAB, TAM, VER).

Eragrostis hirta E. Fourn.

Eragrostis hirta E. Fourn. var. hirta (CHIS, JAL, MÉX, OAX, SLP, TAM, ZAC).

*Eragrostis hirta var. Iongiramea (Swallen) Witherspoon (NL, OAX, SLP, TAM).

Eragrostis hondurensis R.W. Pohl (CHIS, OAX).

Eragrostis hypnoides (Lam.) Britton, Sterns \& Poggenb. (BC, BCS, CAM, CHIS, GTO, GRO, OAX, SLP, SON,

TAB, TAM, VER).

Eragrostis intermedia Hitchc.

*Eragrostis intermedia var. appressa Witherspoon (JAL, ZAC).

Eragrostis intermedia Hitchc. var. intermedia (AGS, BC, CHIH, CHIS, COAH, CDM, DGO, GTO, HGO,

JAL, MÉX, MICH, MOR, NAY, NL, OAX, PUE, QRO, SLP, SON, TAM, TLX, VER).

*Eragrostis intermedia var. oreophila (L.H. Harv.) Witherspoon (BC, BCS, CHIH, HGO, MICH, NL).

Eragrostis japonica (Thunb.) Trin. (OAX).

+Eragrostis lehmanniana Nees $(\mathrm{CHIH}, \mathrm{COAH}, \mathrm{DGO}, \mathrm{NL}, \mathrm{SON})$.

Eragrostis lugens Nees (AGS, BC, BCS, CHIH, CHIS, COAH, CDM, DGO, GTO, GRO, HGO, JAL, MÉX,

MICH, NL, OAX, PUE, QRO, SLP, SON, TAM, TLX, VER, ZAC).

Eragrostis maypurensis (Kunth) Steud. (BC, CHIS, COL, CDM, DGO, GRO, JAL, MÉX, MICH, NAY, OAX, PUE, SIN, TAB, VER, YUC).

Eragrostis mexicana (Hornem.) Link

Eragrostis mexicana (Hornem.) Link subsp. mexicana (AGS, BC, BCS, CHIH, CHIS, COAH, COL, CDM,

DGO, GTO, GRO, HGO, JAL, MEX, MICH, MOR, NAY, NL, OAX, PUE, QRO, SLP, SIN, SON, TAM,

TLX, VER, ZAC).

Eragrostis mexicana subsp. virescens (J. Presl) S.D. Koch \& Sánchez Vega (CHIH).

+Eragrostis minor Host (MÉX, PUE, VER).

Eragrostis palmeri S. Watson (BC, CHIH, COAH, DGO, NL, OAX, SON, TAM, VER, ZAC).

Eragrostis pectinacea (Michx.) Nees

Eragrostis pectinacea var. miserrima (E. Fourn.) Reeder (AGS, BC, BCS, CAM, CHIH, CHIS, COAH, COL, CDM, DGO, GTO, GRO, HGO, JAL, MÉX, MICH, MOR, NAY, NL, OAX, PUE, QRO, QROO, SLP, SIN, SON, TAB, TAM, TLX, VER, YUC, ZAC). 
Eragrostis pectinacea (Michx.) Nees var. pectinacea (AGS, BC, BC, SUR, CAM, CHIH, CHIS, COAH, COL, CDM, DGO, GTO, GRO, HGO, JAL, MÉX, MICH, MOR, NAY, NL, OAX, PUE, QRO, QROO, SLP, SIN, SON, TAB, TAM, TLX, VER, YUC, ZAC).

+Eragrostis pilosa (L.) P. Beauv. (BC, BCS, CHIH, CHIS, COAH, COL, CDM, DGO, GTO, GRO, HGO, JAL, MÉX, MICH, MOR, NAY, NL, OAX, PUE, QRO, SLP, SIN, SON, TAB, TAM, VER).

*Eragrostis plumbea Scribn. ex Beal (AGS, COL, CDM, GTO, JAL, MÉX, MICH, PUE, QRO, SLP, VER, ZAC). Eragrostis polytricha Nees (CHIS, GTO, OAX, PUE, VER).

*Eragrostis pringlei Mattei (CHIH, DGO, GTO, JAL, MICH, ZAC).

Eragrostis prolifera (Sw.) Steud. (CAM, CHIS, COL, GRO, JAL, NAY, OAX, QROO, SIN, TAB, VER, YUC). Eragrostis reptans (Michx.) Nees (BC, BCS, COAH, NL, TAB, TAM, VER).

Eragrostis rufescens Schrad. ex Schult.

Eragrostis rufescens var. mesoamericana Davidse (CHIS, OAX, TAB, VER).

Eragrostis secundiflora J. Presl

Eragrostis secundiflora subsp. oxylepis (Torr.) S.D. Koch (TAM, VER).

Eragrostis secundiflora J. Presl subsp. secundiflora (GRO, OAX).

Eragrostis sessilispica Buckley (CHIH, TAM).

Eragrostis silveana Swallen (COAH, NL, OAX, SLP, TAM).

Eragrostis simpliciflora (J. Presl) Steud. (CHIS).

+Eragrostis spectabilis (Pursh) Steud. (CHIH, COAH, NL, PUE, SLP, TAM).

Eragrostis spicata Vasey (BCS, SON, TAM).

*Eragrostis superba Peyr. (AGS, CHIH, COAH, GTO, MICH, NL, SLP, SON, TAM)..

Eragrostis swallenii Hitchc. (CHIS, GTO, JAL, MÉX, OAX, PUE, QRO, SLP, TAM, VER).

+Eragrostis tenuifolia (A. Rich.) Hochst. ex Steud. (CHIS, OAX, VER, YUC).

+ Eragrostis unioloides (Retz.) Nees ex Steud. (CHIS, TAB).

+Eragrostis viscosa (Retz.) Trin. (BC, BCS, CHIS, CDM, DGO, GRO, JAL, MÉX, MICH, MOR, NAY, OAX, SIN, $T A B, V E R, Y U C)$.

Eremochloa (1/0/0/1; Panicoideae)

+ Eremochloa ophiuroides (Munro) Hack. (BC).

Eriochloa (8/0/0/0; Panicoideae)

Eriochloa acuminata (J. Presl) Kunth

Eriochloa acuminata (J. Presl) Kunth var. acuminata (AGS, BCS, CHIH, COAH, COL, DGO, GTO, GRO, JAL, MICH, MOR, NAY, NL, OAX, PUE, QRO, SLP, SIN, SON).

Eriochloa acuminata var. minor (Vasey) R.B. Shaw $(\mathrm{CHIH}, \mathrm{COAH}, \mathrm{DGO}, \mathrm{JAL}, \mathrm{MÉX}, \mathrm{MICH}, \mathrm{NL}, \mathrm{QRO}$, SLP, SON).

Eriochloa aristata Vasey

Eriochloa aristata Vasey var. aristata $(\mathrm{BC}, \mathrm{CHIH}, \mathrm{CHIS}, \mathrm{COL}, \mathrm{DGO}, \mathrm{GRO}, \mathrm{JAL}, \mathrm{MOR}, \mathrm{NAY}, \mathrm{OAX}, \mathrm{PUE}$, SIN, SON, VER, YUC).

Eriochloa aristata var. boxiana (Hitchc.) R.B. Shaw (BC, CAM, CHIH, CHIS, COL, DGO, GTO, GRO,

JAL, MOR, NAY, OAX, PUE, SIN, SON, VER, YUC).

Eriochloa contracta Hitchc. (CHIH, COAH, DGO, JAL, NAY, NL, OAX, SIN, SON, TAM).

Eriochloa lemmonii Vasey \& Scribn. (CHIH, COAH, COL, DGO, JAL, MÉX, MOR, NAY, SIN, SON).

Eriochloa nelsonii Scribn. \& J.G. Sm.

Eriochloa nelsonii Scribn. \& J.G. Sm. var. nelsonii (CAM, CHIH, CHIS, COL, DGO, GRO, JAL, MÉX,

$\mathrm{MICH}$, MOR, NAY, OAX, PUE, SON).

*Eriochloa nelsonii var. papillosa R.B. Shaw (GRO, JAL, MICH, NAY, PUE).

Eriochloa polystachya Kunth (CHIS).

Eriochloa punctata (L.) Desv. ex Ham. (CHIH, CHIS, COAH, COL, CDM, GTO, GRO, HGO, JAL, MÉX, MOR, NL, OAX, SLP, TAB, TAM, VER, YUC, ZAC).

Eriochloa sericea (Scheele) Munro ex Vasey (COAH, TAM).

Eriochrysis (1/0/0/0; Panicoideae)

Eriochrysis cayennensis P. Beauv. (CHIS, OAX, TAB, VER).

Erioneuron (3/0/0/0; Chloridoideae)

Erioneuron avenaceum (Kunth) Tateoka

Erioneuron avenaceum (Kunth) Tateoka var. avenaceum (AGS, CHIH, COAH, CDM, DGO, GTO, HGO, JAL, MÉX, NL, OAX, PUE, QRO, SLP, TAM, TLX, ZAC).

Erioneuron nealleyi (Vasey) Tateoka (AGS, CHIH, COAH, DGO, GTO, HGO, NL, QRO, SLP, TAM, ZAC).

Erioneuron pilosum (Buckley) Nash

Erioneuron pilosum (Buckley) Nash var. pilosum (AGS, CHIH, COAH, NL, OAX, PUE, QRO, SLP, TAM, ZAC). 
Euclasta (1/0/0/1; Panicoideae)

+Euclasta condylotricha (Hochst. ex Steud.) Stapf (CHIS, COL, GTO, GRO, JAL, MÉX, MICH, NAY, OAX, SIN, VER).

Eustachys (1/0/0/0; Chloridoideae)

Eustachys petraea (Sw.) Desv. (CAM, NL, QROO, TAB, TAM, VER, YUC).

Festuca (30/11/7/0; Pooideae)

**Festuca aequipaleata E. Fourn. (VER).

Festuca aguana E.B. Alexeev (CHIS, GRO).

Festuca amplissima Rupr. (BC, CHIH, COAH, COL, CDM, DGO, GTO, GRO, HGO, JAL, MÉX, MICH, MOR, NAY, NL, OAX, PUE, QRO, SIN, SON, TAB, TAM, TLX, VER).

Festuca arizonica Vasey (COAH, DGO, NL, ZAC).

*Festuca asperella E.B. Alexeev (CDM, MÉX).

**Festuca bajacaliforniana Gonz.-Led. \& S.D. Koch (BC).

**Festuca bidenticulata E.B. Alexeev (VER).

Festuca breviglumis Swallen (AGS, CHIS, DGO, GTO, JAL, MICH, MOR, OAX).

**Festuca callosa (Piper) St.-Yves (PUE).

**Festuca coahuilana Gonz.-Led. \& S.D. Koch (COAH).

*Festuca diclina Darbysh. (CHIH, CHIS).

Festuca hephaestophila Nees ex Steud. (CHIS, CDM, MÉX, NL, PUE, TAM, TLX, VER).

*Festuca hintoniana E.B. Alexeev (COAH, NL, TAM).

*Festuca jaliscana E.B. Alexeev (COL, JAL).

Festuca ligulata Swallen (COAH).

*Festuca livida (Kunth) Willd. ex Spreng. (CHIS, CDM, MÉX, PUE, VER).

*Festuca lugens (E. Fourn.) Hitchc. ex Hern.-Xol. (CHIH, CHIS, CDM, GTO, HGO, JAL, MÉX, MICH, MOR, NAY, OAX, PUE, QRO, SLP, TAM, TLX, VER).

*Festuca orizabensis E.B. Alexeev (CHIS, CDM, DGO, JAL, MÉX, MICH, MOR, PUE, VER).

*Festuca pinetorum Swallen (COAH, NL, TAM).

*Festuca pringlei St.-Yves (AGS, CHIH, DGO, JAL).

*Festuca roblensis Gonz.-Led. (GTO, GRO, ZAC).

Festuca rosei Piper (AGS, CHIS, CDM, DGO, GRO, JAL, MÉX, MICH, MOR, PUE, QRO, SLP, TAM, TLX, VER, ZAC).

Festuca rubra L. (CHIHG, COAH, CDM, DGO, MÉX, NL, PUE, QRO, TAM, VER).

**Festuca rzedowskiana E.B. Alexeev (MÉX).

Festuca subulata Trin. (CHIS).

${ }^{* *}$ Festuca tancitaroensis Gonz.-Led. \& S.D. Koch (MICH).

Festuca thurberi Vasey (COAH, NL, TAM).

Festuca tolucensis Kunth (CHIH, CHIS, CDM, DGO, JAL, MÉX, MICH, MOR, PUE, SLP, TLX, VER).

*Festuca valdesii Gonz.-Led. \& S.D. Koch (COAH, NL, TAM).

Festuca willdenowiana Schult. \& Schult. f. (CHIS, CDM, GRO, JAL, MÉX, OAX, PUE, VER).

Gastridium (1/0/0/1; Pooideae)

+Gastridium ventricosum (Gouan) Schinz \& Thell. (BC).

Glyceria (4/0/0/1; Pooideae)

Glyceria borealis (Nash) Batch. (CHIH).

+Glyceria fluitans (L.) R. Br. (DGO, GTO, HGO, MÉX, MICH, QRO, SLP).

Glyceria septentrionalis Hitchc. (MÉX, QRO, SLP).

Glyceria striata (Lam.) Hitchc.

Glyceria striata var. mexicana Kelso (CHIS, CDM, HGO, MÉX, MOR, OAX).

Glyceria striata (Lam.) Hitchc. var. striata (CHIH, DGO).

Gouinia (5/2/1/0; Chloridoideae)

**Gouinia isabelensis J.J. Ortíz (NAY).

Gouinia latifolia (Griseb.) Vasey

Gouinia latifolia var. guatemalensis (Hack.) J.J. Ortíz (CAM, OAX, QRO, QROO, YUC).

*Gouinia mexicana (Scribn.) Vasey (QRO, SLP).

*Gouinia papillosa Swallen (QROO, TAM, YUC).

Gouinia virgata (J. Presl) Scribn.

*Gouinia virgata var. robusta J.J. Ortíz (GRO, JAL, MICH, MOR, ZAC).

Gouinia virgata (J. Presl) Scribn. var. virgata (CAM, CHIS, COL, DGO, GRO, JAL, MICH, NAY, OAX,

QRO, QROO, SLP, SIN, TAM, VER, YUC, ZAC). 
Guadua (8/2/1/1; Bambusoideae)

Guadua aculeata Rupr. ex E. Fourn. (CHIS, PUE, VER).

Guadua amplexifolia J. Presl (CAM, CHIS, PUE, QROO, TAB, VER).

+Guadua angustifolia Kunth (TAB, VER).

*Guadua inermis Rupr. ex E. Fourn. (CAM, CHIS, OAX, TAB, VER).

Guadua longifolia (E. Fourn.) R.W. Pohl (CAM, CHIS, TAB, VER).

Guadua paniculata Munro (CHIS, GRO, JAL, NAY, OX, SLP).

**Guadua tuxtlensis Londoño \& Ruíz-Sánchez (VER).

${ }^{*}$ Guadua velutina Londoño \& L.G. Clark (HGO, PUE, SLP, TAB, VER).

Gymnopogon (1/0/0/0; Chloridoideae)

Gymnopogon spicatus (Spreng.) Kuntze

Gymnopogon spicatus fo. spicatus (VER).

Gynerium (1/0/0/1; Panicoideae)

+Gynerium sagittatum (Aubl.) P. Beauv. (CAM, CHIS, OAX, PUE, SLP, TAB, VER).

Hainardia (1/0/0/1; Pooideae)

+Hainardia cylindrica (Willd.) Greuter (BC).

Hemarthria (1/0/0/0; Panicoideae)

Hemarthria altissima (Poir.) Stapf \& C.E. Hubb. (CAM, CHIS, COAH, GRO, MÉX, MOR, NL, PUE, SLP, TAB, TAM, VER, YUC).

Hesperostipa (3/0/1/0; Pooideae)

Hesperostipa comata (Trin. \& Rupr.) Barkworth (BC).

Hesperostipa neomexicana (Thurb.) Barkworth (CHIH, DGO).

**Hesperostipa saxicola (Hitchc.) Valdés-Reyna \& Barkworth (PUE).

Heteropogon (2/0/0/0; Panicoideae)

Heteropogon contortus (L.) P. Beauv. ex Roem. \& Schult. (AGS, BC, BCS, CAM, CHIH, CHIS, COAH, COL, CDM, DGO, GTO, GRO, HGO, JAL, MÉX, MICH, MOR, NAY, NL, OAX, PUE, QRO, QROO, SLP, SIN, SON, TAB, TAM, VER, YUC, ZAC).

Heteropogon melanocarpus (Elliott) Benth. (AGS, BC, BCS, CHIH, CHIS, COAH, COL, CDM, DGO, GTO, GRO, HGO, JAL, MÉX, MICH, MOR,. NAY, NL, OAX, PUE, QRO, SLP, SIN, SON, TAM, VER, ZAC).

Hilaria (9/2/2/0; Chloridoideae)

**Hilaria annua Reeder \& C. Reeder (COL).

Hilaria belangeri (Steud.) Nash

Hilaria belangeri (Steud.) Nash var. belangeri (AGS, BC, BCS, CHIH, COAH, GTO, GRO, MÉX, MICH, MOR, NL, OAX, PUE, QRO, SON, TAM.

Hilaria belangeri var. longifolia (Vasey) Hitchc. (BCS, SON).

Hilaria cenchroides Kunth (AGS, BC, BCS, CHIH, CHIS, COAH, CDM, DGO, GTO, GRO, HGO, JAL, MÉX, MICH, MOR, NL, OAX, PUE, QRO, SLP, SON, TAM, TLX, VER, ZAC).

*Hilaria ciliata (Scribn.) Nash (BC, BCS, CHIH, COL, GTO, GRO, JAL, MICH, NAY, OAX, QRO, SLP, SON, TAM, VER).

*Hilaria hintonii Sohns (GRO, MÉX, MICH, MOR, QRO).

Hilaria mutica (Buckley) Benth. (BCS, CHIH, COAH, DGO, NL, SON, ZAC).

Hilaria rigida (Thurb.) Benth. ex Scribn. (BC, $\mathrm{CHIH}, \mathrm{SON}$ ).

**Hilaria semplei Sohns (MICH).

Hilaria swallenii Cory (CHIH, COAH, DGO, NL, SLP, ZAC).

Holcus (1/0/0/1; Pooideae)

+Holcus lanatus L. (BC, CHIS, CHIH, MÉX, MICH, PUE, VER).

Homolepis (2/0/0/0; Panicoideae)

Homolepis aturensis (Kunt) Chase (CHIS, OAX, PUE, TAB, VER).

Homolepis glutinosa (Sw.) Zuloaga \& Soderstr. (CHIS, HGO, OAX, PUE, TAM, VER).

Hopia (1/0/0/0; Panicoideae)

Hopia obtusa (Kunth) Zuloaga and Morrone (AGS, CHIH, COAH, DGO, GTO, HGO, JAL, MÉX, MICH, NL, PUE, QRO, SLP, SON, ZAC).

Hordeum (7/0/0/1; Pooideae)

Hordeum arizonicum Covas (BC, DGO, SON). 
Hordeum brachyantherum Nevski (BC).

Hordeum depressum (Scribn. \& J.G. Sm.) Rydb. (BC).

Hordeum jubatum L. (AGS, BC, COAH, CDM, DGO, HGO, JAL, MÉX, PUE, QRO, SLP, ZAC).

Hordeum murinum $\mathrm{L}$.

+Hordeum murinum subsp. glaucum (Steud.) Tzvelev $(\mathrm{CHIH})$.

Hordeum pusillum Nutt. (BC).

+Hordeum vulgare L. (AGS, BC, CHIH, CHIS, COAH, CDM, HGO, JAL, MÉX, MICH, MOR, NL, OAX, PUE, SLP, SON, TAB, TAM, VER, ZAC).

Hymenachne (1/0/0/0; Panicoideae)

Hymenachne amplexicaulis (Rudge) Nees (CAMS, CHIS, COL, GTO, GRO, JAL, MICH, NAY, OAX, QROO, SIN, TAB, TAM, VER, YUC).

Hyparrhenia (3/0/0/1; Panicoideae)

Hyparrhenia bracteata (Humb. \& Bonpl. ex Willd.) Stapf (CHIS, OAX, VER).

+Hyparrhenia hirta (L.) Stapf (CHIS, NL, OAX, SLP, YUC).

Hyparrhenia rufa (Nees) Stapf

Hyparrhenia rufa (Nees) Stapf var. rufa (CAM, CHIS, COL, GRO, JAL, MÉX, MICH, MOR, NAY, QROO, SIN, TAB, TAM, VER, YUC).

Hyperthelia (1/0/0/01; Panicoideae)

+Hyperthelia dissoluta (Nees ex Steud.) Clayton (CHIS, COL, GRO, JAL, MÉX, MICH, NAY, SIN, VER, YUC).

Ichnanthus (6/0/0/0: Panicoideae)

Ichnanthus calvescens (Nees ex Trin.) Döll

Ichnanthus calvescens var. scabrior Döll (TAB).

Ichnanthus lanceolatus Scribn. \& J.G. Sm. (CAM, CHIS, QROO, TAB, YUC).

Ichnanthus nemoralis (Schrad.) Hitchc. \& Chase (QROO, YUC).

Ichnanthus nemorosus (Sw.) Döll (CAM, CHIS, GRO, HGO, JAL, OAX, PUE, QRO, SLP, VER).

Ichnanthus pallens (Sw.) Munro ex Benth.

Ichnanthus pallens var. major (Nees) Stieber (VER).

Ichnanthus pallens (Sw.) Munro ex Benth. var. pallens (CAM, CHIS, HGO, OAX, PUE, QROO, SLP, TAB, VER).

Ichnanthus tenuis (J. Presl \& C. Presl) Hitchc. \& Chase (CHIS, OAX, PUE, QROO, TAB, VER, YUC).

Imperata (3/0/0/0; Panicoideae)

Imperata brasiliensis Trin. (CAM, CHIS, JAL, OAX, QROO, TAB, VER, YUC).

Imperata contracta (Kunth) Hitchc. (CHIS, TAB, VER).

Imperata cylindrica (L.) P. Beauv. (CHIS).

Isachne (3/0/0/0; Micrairoideae)

Isachne arundinacea (Sw.) Griseb. (CHIS, GRO, HGO, OAX, PUE, VER).

Isachne polygonoides (Lam.) Döll. (CHIS, GRO).

Isachne pubescens Swallen (CHIS, VER).

Ischaemum (2/0/0/1; Panicoideae)

Ischaemum latifolium (Spreng.) Kunth (CAM, CHIS, OAX, PUE, QROO, VER, YUC).

+Ischaemum rugosum Salisb. (CAM, GRO, JAL, MICH, NAY).

Ixophorus (1/0/0/0; Panicoideae)

Ixophorus unisetus (J. Presl) Schltdl. (AGS, CAM, CHIS, COL, GTO, GRO, JAL, MÉX, MICH, MOR, NAY, NL, OAX, PUE, SLP, SIN, TAM, VER).

Jarava (2/0/0/0; Pooideae)

Jarava ichu Ruíz \& Pav. (COAH, OAX, SLP, VER).

Jarava speciosa (Trin. \& Rupr.) Peñailillo (BC, BCS).

Jouvea (2/0/0/0; Chloridoideae)

Jouvea pilosa (J. Presl) Scribn. (BCS, CHIS, COL, GRO, JAL, MICH, NAY, OAX, SIN, SON, TAM, YUC).

Jouvea straminea E. Fourn. (CHIS, GRO, JAL, NAY, OAX, SIN).

Kalinia (1/0/0/0; Chloridoideae)

Kalinia obtusiflora (E. Fourn.) H.L. Bell \& Columbus (CHIH, COAH, CDM, GTO, JAL, MÉX, MICH, NL, OAX, SON, VER, ZAC). 
Koeleria (2/0/0/0; Pooideae) Koeleria macrantha (Ledeb.) Schult. (SON).

Koeleria pyramidata (Lam.) P. Beauv. (BC, BCSUR, CHIH, COAH, COL, CDM, DGO, GTO, JAL, MÉX, NL, OAX, SLP, SON, TAM, TLX, VER, ZAC).

Lachnagrostis (1/0/0/0; Pooideae)

Lachnagrostis filiformis (G. Forst.) Trin. (CHIS)

Lamarckia $(1 / 0 / 0 / 1 ;$ Pooideae)

+Lamarckia aurea (L.) Moench (BC, BCS).

Lasiacis (13/0/0/0; Panicoideae)

Lasiacis divaricata (L.) Hitchc.

Lasiacis divaricata (L.) Hitchc. var. divaricata (BCS, CAM, CHIS, COL, GRO, JAL, MÉX, MICH, MOR,

NAY, NL, OAX, PUE, QROO, SLP, SIN, TAB, TAM, VER, YUC).

Lasiacis divaricata var. leptostachya (Hitchc.) Davidse (CHIS, OAX, TAB, VER).

Lasiacis grisebachii (Nash) Hitchc.

Lasiacis grisebachii (Nash) Hitchc. var. grisebachii (CAM, CHIS, HGO, OAX, PUE, QROO, SLP, TAB,

VER, YUC).

Lasiacis linearis Swallen (CHIS).

Lasiacis nigra Davidse (AGS, CHIS, COL, CDM, DGO, GTO, GRO, HGO, JAL, MÉX, MICH, MOR, NAY, NL, OAX, PUE, QRO, SLP, TAB, TAM, VER, ZAC).

Lasiacis oaxacensis (Steud.) Hitchc.

Lasiacis oaxacensis var. maxonii (Swallen) Davidse (CHIS).

Lasiacis oaxacensis (Steud.) Hitchc. var. oaxacensis (CHIS, JAL, MÉX, NAY, OAX, TAB, VER).

Lasiacis procerrima (Hack.) Hitchc. (CHIS, COL, DGO, GRO, JAL, MÉX, MICH, NAY, OAX, PUE, SIN, TAB, TAM, VER).

Lasiacis rhizophora (E. Fourn.) Hitchc. (CHIS, GRO, MOR, MICH, OAX, PUE, VER).

Lasiacis rugelii (Griseb.) Hitchc.

Lasiacis rugelii var. pohlii Davidse (CHIS).

Lasiacis rugelii (Griseb.) Hitchc. var. rugelii (CAM, CHIS, OAX, QROO, SLP, TAB, TAM, VER, YUC). Lasiacis ruscifolia (Kunth) Hitchc. (BC, BCS, CAM, CHIH, CHIS, COL, DGO, GRO, HGO, JAL, MÉX, MICH, MOR, NAY, NL, OAX, PUE, QRO, QROO, SLP, SIN, SON, TAB, TAM, VER, YUC).

Lasiacis scabrior Hitchc. (CHIS, OAX, VER).

Lasiacis sloanei (Griseb.) Hitchc. (CAM, CHIS, OAX, QRO, QROO, SLP, TAB, TAM, VER, YUC).

Lasiacis sorghoidea (Desv. ex Ham.) Hitchc. \& Chase (CHIS, JAL, MÉX, OAX, SLP, TAB, VER).

Lasiacis standleyi Hitchc. (CHIS).

Leersia (4/0/0/0; Oryzoideae)

Leersia hexandra Sw. (CAM, CHIH, CHIS, COAH, COL, CDM, DGO, GTO, GRO, JAL, MÉX, MICH, MOR, NAY, OAX, PUE, SLP, TAB, TAM, VER, YUC, ZAC).

Leersia ligularis Trin.

Leersia ligularis var. breviligulata (Prod.) Pyrah (CAM, CHIS, HGO, MÉX, NL, OAX, PUE, QRO, SLP, TAB,

TAM, VER, YUC).

*Leersia ligularis Trin. var. ligularis (CHIS, PUE, QRO, SLP, TAM, VER).

Leersia monandra Sw. (CAM, CHIS, COAH, NL, OAX, PUE, QRO, SLP, TAB, TAM, VER, YUC).

Leersia oryzoides (L.) Sw. (COAH, NL, SLP, TAM).

Leptochloa (3/0/0/0; Chloridoideae)

Leptochloa crinita (Lag.) P.M. Peterson \& N. Snow (BCS, CHIH, COAH, DGO, SLP, SON).

Leptochloa pluriflora (E. Fourn.) P.M. Peterson \& N. Snow (CAM, CHIH, COAH, DGO, JAL, MICH, NL, OAX, PUE, SLP, TAM, YUC).

Leptochloa virgata (L.) P. Beauv. (BC, BCS, CAM, CHIH, CHIS, COL, GTO, JAL, MICH, NAY, OAX, PUE, QROO, SLP, SON, TAB, TAM, VER, YUC).

Leymus (2/0/0/0; Pooideae)

Leymus condensatus (J. Presl) Á. Löve (COAH).

Leymus triticoides (Buckley) Pilg. (BC).

Limnodea (1/0/0/0; Pooideae)

Limnodea arkansana (Nutt.) L.H. Dewey (COAH, TAM).

Lithachne (1/0/0/0; Bambusoideae)

Lithachne pauciflora (Sw.) P. Beauv. (CAM, CHIS, JAL, OAX, PUE, QROO, SLP, TAB, TAM, VER, YUC). 
Lolium (3/0/0/3; Pooideae)

+Lolium multiflorum Lam. (AGS, BC, CHIH, CHIS, COAH, CDM, DGO, GTO, JAL, MÉX, NL, OAX, PUE, SLP, SON, TLX, VER, ZAC).

+Lolium perenne L. (AGS, BC, CHIH, CHIS, COAH, CDM, DGO, GTO, JAL, MÉX, MICH, NL, OAX, PUE, SON, TAM, VER, ZAC).

+Lolium temulentum L. (AGS, BC, JAL, OAX, PUE, SLP, VER, ZAC).

Luziola (4/0/0/0; Oryzoideae)

Luziola fluitans (Michx.) Terrell \& H. Rob.

Luziola fluitans (Michx.) Terrell \& H. Rob. var. fluitans (AGS, GTO, HGO, JAL, MÉX).

Luziola gracillima Prod. (GTO, JAL, SIN, SON).

Luziola peruviana Juss. ex J.F. Gmel. (CAM, CHIS, CDM, HGO, JAL, MÉX, MICH, OAX, QRO, TAB, VER). Luziola subintegra Swallen (CHIS, TAB, VER).

Melica (5/0/0/0; Pooideae)

Melica frutescens Scribn. (BC, BCS).

Melica imperfecta Trin. (BC, BCS).

Melica montezumae Piper (CHIH, COAH, NL).

Melica nitens (Nutt.) Nutt. ex Piper (COAH, NL, TAM).

Melica porteri Scribn. (CHIH, COAH).

Melinis (2/0/0/2; Panicoideae)

+Melinis minutiflora P. Beauv. (CAM, CHIS, COL, GTO, GRO, HGO, JAL, MEX, MICH, MOR, OAX, PUE, QRO, QROO, TAB, TAM, TLX, VER, YUC).

+Melinis repens (Willd.) Zizka (CAM, CHIH, DGO, OAX, QROO, VER, YUC, ZAC).

Merostachys (1/0/1/0; Bambusoideae)

**Merostachys mexicana Ruiz-Sanchez \& L.G. Clark (CHIS).

Mesosetum (4/0/0/0; Panicoideae)

Mesosetum blakei Swallen (CHIS, TAB, VER).

Mesosetum filifolium F.T. Hubb. (OAX).

Mesosetum pittieri Hitchc. (CHIS, OAX, VER).

Mesosetum stoloniferum Swallen (CHIS, TAB).

Metcalfia (1/1/0/0; Pooideae)

*Metcalfia mexicana (Scribn.) Conert (CHIS, COAH, HGO, MÉX, NL, OAX, PUE, QRO, SLP, TAM, ZAC).

Microchloa (1/0/0/0; Chloridoideae)

Microchloa kunthii Desv. (AGS, BC, BCS, CHIH, CHIS, COAH, COL, CDM, DGO, GTO, GRO, HGO, JAL, MÉX, MICH, MOR, NAY, OAX, PUE, QRO, SLP, SIN, SON, TLX, VER, ZAC).

Miscanthus (1/0/0/1; Panicoideae)

+Miscanthus sinensis Andersson (TAB, VER).

Mnesithea (4/0/0/1; Panicoideae)

Mnesithea aurita (Steud.) de Koning \& Sosef (CHIS, CDM, JAL, MÉX, MICH, NAY, PUE, TAB, VER).

+Mnesithea granularis (L.) de Koning \& Sosef (BCS, CAM, CHIH, CHIS, COL, DGO, GTO, GRO, JAL, MÉX, MICH, MOR, NAY, OAX, PUE, SIN, SON, TAB, TAM, VER, YUC, ZAC).

Mnesithea ramosa (E. Fourn.) de Koning \& Sosef (VER, ZAC).

Mnesithea subgibbosa (Winkl. ex Hack.) de Koning \& Sosef (CHIS).

Morronea (6/0/0/0; Panicoideae)

Morronea arundinariae (Trin. ex E. Fourn.) Zuloaga and Scataglini (VER).

Morronea cayoensis (Swallen) Zuloaga and Scataglini (CHIS).

Morronea guatemalensis (Swallen) Zuloaga and Scataglini (CHIS, VER).

Morronea incumbens (Swallen) Zuloaga and Scataglini (CHIS).

Morronea parvig/umis (Hack.) Zuloaga and Scataglini (VER).

Morronea trichidiachnis (Döll) Zuloaga and Scataglini (VER).

Muhlenbergia (127/35/16/0; Chloridoideae)

**Muhlenbergia aguascalientensis Y. Herrera \& De la Cerda-Lemus (AGS).

*Muhlenbergia alamosae Vasey (AGS, BC, BCS, CHIH, DGO, GTO, JAL, MÉX, MOR, PUE, SIN, SON, ZAC).

Muhlenbergia alopecuroides (Griseb.) P.M. Peterson \& Columbus (BC, CHIH, COAH, DGO, SLP, SON).

*Muhlenbergia annua (Vasey) Swallen (CHIH, DGO, SON). 
Muhlenbergia appressa C.O. Goodd. (BC, BCS).

Muhlenbergia arenacea (Buckley) Hitchc. (CHIH, COAH, SLP, SON, ZAC).

Muhlenbergia arenicola Buckley $(\mathrm{CHIH}, \mathrm{COAH}, \mathrm{DGO}, \mathrm{NAY}, \mathrm{NL}, \mathrm{SLP}, \mathrm{SON}, \mathrm{TAM}, \mathrm{ZAC})$.

**Muhlenbergia argentea Vasey $(\mathrm{CHIH})$.

Muhlenbergia arizonica Scribn. (BC, BCS, CHIH, DGO, SIN, SON).

Muhlenbergia arsenei Hitchc. (BC).

Muhlenbergia articulata Scribn. (CHIH, GTO, GRO, HGO, QRO, SLP, SON).

Muhlenbergia asperifolia (Nees \& Meyen ex Trin.) Parodi (BC, CHIH, COAH, DGO, JAL, SLP, SON).

Muhlenbergia beyrichiana Kunth (CHIS).

*Muhlenbergia biloba Hitchc. (CHIH, DGO, SON).

**Muhlenbergia brandegeei C. Reeder (BCS).

*Muhlenbergia brevifolia Scribn. ex Beal (DGO, GTO, JAL, NAY).

Muhlenbergia breviligula Hitchc. (CHIS).

Muhlenbergia brevis C.O. Goodd. (CHIH, CDM, DGO, JAL, MÉX, SLP, ZAC).

*Muhlenbergia breviseta Griseb. ex E. Fourn. (GRO, JAL, MÉX, MICH, NAY, VER).

*Muhlenbergia brevivaginata Swallen (AGS, DGO, JAL, NAY, SIN, ZAC).

Muhlenbergia capillaris (Lam.) Trin. (OAX, QROO, VER, YUC).

*Muhlenbergia capillipes (M.E. Jones) P.M. Peterson \& Annable (CHIH, SON).

Muhlenbergia cenchroides (Humb. \& Bonpl. Ex Willd.) P.M. Peterson (BC, BCS, CHIH, CHIS, COL, CDM, DGO, GTO, GRO, HGO, JAL, MÉX, MICH, MOR, NAY, OAX, PUE, QRO, SLP, SIN, SON, TLX, VER, ZAC). Muhlenbergia ciliata (Kunth) Trin. (AGS, BC, BCS, CHIH, CHIS, COL, CDM, DGO, GTO, GRO, HGO, JAL, MÉX, MICH, MOR, NAY, OAX, PUE, QRO, SIN, SON, VER, ZAC).

Muhlenbergia crispiseta Hitchc. (AGS, CHIH, DGO, GTO, JAL, MÉX, NAY, SLP, SIN, SON, ZAC).

**Muhlenbergia cualensis Y. Herrera \& P.M. Peterson (JAL).

**Muhlenbergia decumbens Swallen $(\mathrm{CHIH})$

Muhlenbergia depauperata Scribn. (AGS, CHIH, COAH, CDM, DGO, GTO, HGO, JAL, MÉX, NL, OAX, PUE, $\mathrm{QRO}, \mathrm{SLP}, \mathrm{VER}, \mathrm{ZAC})$.

Muhlenbergia distichophylla (J. Presl) Kunth (CHIH, CHIS, COL, GRO, JAL, MÉX, MICH, OAX, PUE, TAM). Muhlenbergia diversiglumis Trin. (CHIH, CHIS, DGO, HGO, JAL, MÉX, MICH, MOR, NAY, SIN, SON, ZAC). Muhlenbergia dubia E. Fourn. (AGS, CHIH, COAH, CDM, DGO, GTO, HGO, JAL, MÉX, MICH, NL, OAX, PUE, QRO, SLP, TAM, TLX, VER, ZAC).

Muhlenbergia dumosa Scribn. ex Vasey (BC, BCS, CHIH, DGO, JAL, MÉX, MICH, OAX, PUE, QRO, SON, VER, ZAC).

*Muhlenbergia durangensis Y. Herrera (CHIH, DGO, ZAC).

Muhlenbergia elongata Scribn. ex Beal (CHIH, HGO, SON).

Muhlenbergia eludens $\mathrm{C}$. Reeder $(\mathrm{CHIH}, \mathrm{DGO}, \mathrm{SIN}, \mathrm{SON})$.

Muhlenbergia emersleyi Vasey (AGS, BC, BCS, CHIH, CHIS, COAH, CDM, DGO, GTO, GRO, HGO, JAL,

MÉX, MICH, MOR, NAY, NL, OAX, PUE, QRO, SLP, SIN, SON, TAM, TLX, VER, ZAC).

*Muhlenbergia eriophylla Swallen (AGS, DGO, GTO, MÉX, MICH, ZAC).

Muhlenbergia filiculmis Vasey (DGO).

Muhlenbergia filiformis (Thurb. ex S. Watson) Rydb. (AGS, BC, DGO, OAX).

*Muhlenbergia flavida Vasey (CHIH, DGO, HGO, JAL, MICH, MOR, NAY, SIN, SON).

*Muhlenbergia flaviseta Scribn. (CHIH, DGO, ZAC).

Muhlenbergia fragilis Swallen (AGS, BC, BCS, CHIH, CHIS, COAH, DGO, GTO, GRO, JAL, MICH, MOR,

NAY, NL, OAX, PUE, QRO, SLP, SIN, SON, TAM, VER, ZAC).

*Muhlenbergia gigantea (E. Fourn.) Hitchc. (CHIH, CHIS, DGO, GTO, GRO, HGO, JAL, MÉX, MICH, OAX,

PUE, SLP, SIN, VER).

Muhlenbergia glauca (Nees) B.D. Jacks. (AGS, CHIH, COAH, CDM, DGO, GTO, HGO, JAL, MÉX, NL, PUE, SLP, SON, ZAC).

*Muhlenbergia grandis Vasey (JAL, NAY, SIN, SON).

*Muhlenbergia gypsophila Reeder \& C. Reeder (COAH, NL, QRO, SLP).

**Muhlenbergia hintonii Swallen (MÉX).

Muhlenbergia imperfecta (Nash) Columbus (CHIH, DGO, MÉX, OAX, SLP, SON, VER).

Muhlenbergia implicata (Kunth) Trin. (AGS, BC, CHIH, CHIS, COL, CDM, DGO, GTO, GRO, HGO, JAL, MÉX, MICH, MOR, NAY, OAX, PUE, QRO, TLX, VER, ZAC).

**Muhlenbergia iridifolia Soderstr. (JAL).

**Muhlenbergia jaime-hintonii P.M. Peterson \& Valdés-Reyna (NL).

**Muhlenbergia jaliscana Swallen (JAL).

**Muhlenbergia laxa Hitchc. (VER).

*Muhlenbergia ligulata (E. Fourn.) Scribn. \& Merr. (AGS, CHIH, CDM, DGO, GTO, JAL, MÉX, SLP, ZAC).

Muhlenbergia lindheimeri Hitchc. $(\mathrm{CHIH}, \mathrm{COAH}, \mathrm{NL})$.

*Muhlenbergia longiglumis Vasey (AGS, DGO, GTO, JAL, MÉX, MICH, ZAC).

Muhlenbergia longiligula Hitchc. (CHIH, DGO, JAL, PUE, SON).

*Muhlenbergia lucida Swallen (CHIH, GTO, MÉX, SON). 
Muhlenbergia macroura (Kunth) Hitchc. (AGS, CHIH, CHIS, COAH, CDM, DGO, GTO, HGO, JAL, MÉX, MICH, MOR, OAX, PUE, SLP, TLX, VER).

**Muhlenbergia majalcensis P.M. Peterson (CHIH).

*Muhlenbergia michisensis Y. Herrera \& P.M. Peterson (DGO, GRO).

Muhlenbergia microsperma (DC.) Kunth (AGS, BC, BCS, CHIH, CHIS, COAH, DGO, GTO, GRO, HGO, JAL, MÉX, MICH, MOR, NAY, NL, OAX, PUE, QRO, SLP, SIN, SON, TAM, TLX, VER, ZAC).

Muhlenbergia minutissima (Steud.) Swallen (AGS, BC, CHIH, CDM, DGO, GTO, HGO, JAL, MÉX, MICH, MOR, NAY, OAX, PUE, QRO, SLP, SIN, SON, TAM, TLX, VER, ZAC).

Muhlenbergia montana (Nutt.) Hitchc. (AGS, CHIH, CHIS, COAH, CDM, DGO, GTO, HGO, JAL, MÉX, MICH, MOR, OAX, PUE, QRO, SON, TLX, ZAC).

*Muhlenbergia mucronata (Kunth) Trin. (CHIH, DGO, GTO, MÉX, OAX, SIN, ZAC).

*Muhlenbergia mutica (Rupr. ex E. Fourn.) Hitchc. (CHIS, PUE, VER).

Muhlenbergia nigra Hitchc. (CHIS, CDM, GTO, JAL, MÉX, MICH, MOR, OAX, PUE, TLX, VER).

Muhlenbergia orophila Swallen (CDM, MÉX, MOR, PUE).

Muhlenbergia palmeri Vasey (CHIH, DGO, MICH, SON).

Muhlenbergia paniculata (Nutt.) Columbus (COAH, TAM).

Muhlenbergia pauciflora Buckley (BC, $\mathrm{CHIH}, \mathrm{COAH}, \mathrm{DGO}, \mathrm{JAL}, \mathrm{NL}, \mathrm{SON})$.

Muhlenbergia pectinata C.O. Goodd. (CHIH, DGO, GRO, JAL, MÉX, MICH, MOR, NAY, OAX, SIN, SON, ZAC).

Muhlenbergia pereilema P.M. Peterson (AGS, BC, BCS, CHIH, CHIS, DGO, GRO, JAL, MÉX, MICH, MOR, NAY, OAX, PUE, QRO, SIN, VER).

Muhlenbergia peruviana (P. Beauv.) Steud. (AGS, CHIH, CHIS, CDM, DGO, GTO, HGO, JAL, MÉX, MICH, MOR, OAX, PUE, QRO, SLP, SIN, TLX, VER, ZAC).

Muhlenbergia phalaroides (Kunth) P.M. Peterson (AGS, BCS, CHIH, CHIS, COAH, COL, CDM, DGO, GTO, HGO, JAL, MÉX, MICH, MOR, NAY, NL, OAX, PUE, QRO, SLP, TAM, TLX, VER, ZAC).

Muhlenbergia phleoides (Kunth) Columbus (AGS, BC, BCS, CHIH, COAH, CDM, DGO, GTO, HGO, JAL, MÉX, MICH, MOR, NAY, NL, OAX, PUE, QRO, SLP, SON, TAM, TLX, ZAC).

*Muhlenbergia pilosa P.M. Peterson, Wipff \& S.D. Jones (GRO, MÉX).

Muhlenbergia plumbea (Trin.) Hitchc. (D.F, DGO, HGO, MÉX, PUE, TLX, ZAC).

Muhlenbergia plumiseta Columbus (CHIS, COL, JAL, MÉX, MICH, MOR, NAY, OAX, VER).

Muhlenbergia polycaulis Scribn. (AGS, BC, BCS, CHIH, COAH, DGO, GTO, HGO, JAL, MÉX, NAY, QRO,

SLP, SIN, SON, ZAC).

Muhlenbergia porteri Scribn. ex Beal (BC, CHIH, COAH, DGO, NL, SLP, SON, TAM, ZAC).

*Muhlenbergia pubescens (Kunth) Hitchc. (AGS, CHIH, COAH, CDM, DGO, GTO, HGO, JAL, MÉX, MICH, NAY, NL, OAX, PUE, SLP, TLX, ZAC).

*Muhlenbergia pubigluma Swallen $(\mathrm{CHIH}, \mathrm{COAH}, \mathrm{NL}, \mathrm{TAM}, \mathrm{ZAC})$.

*Muhlenbergia purpusii Mez (CHIH, COAH, NL, SLP, TAM).

Muhlenbergia quadridentata (Kunth) Trin. (AGS, CHIH, CHIS, COL, CDM, DGO, GTO, GRO, HGO, JAL, MÉX, MICH, MOR, NL, OAX, PUE, QRO, SLP, SON, TLX, VER, ZAC).

Muhlenbergia racemosa (Michx.) Britton (COAH, NL, TAM).

Muhlenbergia ramulosa (Kunth) Swallen (BC, BCS, CHIH, CHIS, CDM, DGO, GTO, GRO, HGO, JAL, MÉX, MICH, MOR, OAX, PUE, QRO, SLP, SIN, SON, TAM, TLX, VER).

*Muhlenbergia reederorum Soderstr. (DGO, JAL, MICH).

Muhlenbergia repens (J. Presl) Hitchc. (AGS, BC, BCS, CHIH, CHIS, COAH, CDM, DGO, GTO, GRO, HGO, JAL, MÉX, MOR, NL, OAX, PUE, QRO, SLP, SON, TAM, TLX, VER, ZAC).

Muhlenbergia reverchonii Vasey \& Scribn. (COAH, NL, PUE, TAM).

Muhlenbergia richardsonis (Trin.) Rydb. (BC, CHIH, CDM, DGO, HGO, PUE, SLP, SON).

Muhlenbergia rigens (Benth.) Hitchc. (AGS, BC, BCS, CHIH, COAH, DGO, JAL, MICH, NL, PUE, SON, ZAC). Muhlenbergia rigida (Kunth) Kunth (AGS, BC, BCS, CHIH, CHIS, COAH, CDM, DGO, GTO, GRO, HGO, JAL, MÉX, MICH, MOR, NAY, NL, OAX, PUE, QRO, SLP, SON, TAM, TLX, VER, ZAC).

Muhlenbergia robusta (E. Fourn.) Hitchc. (AGS, CHIH, CHIS, COL, CDM, DGO, GTO, GRO, HGO, JAL, MÉX, MICH, MOR, NAY, NL, OAX, PUE, QRO, SLP, SIN, VER, ZAC).

${ }^{*}$ Muhlenbergia schmitzii Hack. (CHIH, HGO, MÉX, MICH).

Muhlenbergia schreberi J.F. Gmel. (HGO, PUE, QRO, TLX, VER).

*Muhlenbergia scoparia Vasey (CHIH, DGO, JAL, MICH, NAY, SIN, SON, ZAC).

*Muhlenbergia seatonii Scribn. (HGO, PUE, TLX).

Muhlenbergia setarioides E. Fourn. (CHIS, PUE, VER).

Muhlenbergia setifolia Vasey (CHIH, COAH, DGO, GTO, NL, SLP, TAM).

*Muhlenbergia shepherdii (Vasey) Swallen (CHIH, DGO, SON).

Muhlenbergia sinuosa Swallen (CHIH, GRO, SON).

**Muhlenbergia solisii (G.A. Levin) P.M. Peterson (COL).

**Muhlenbergia spatha Columbus (SLP).

*Muhlenbergia speciosa Vasey (CHIH, DGO, JAL, MICH, NAY, SIN, ZAC).

Muhlenbergia spiciformis Trin. (CHIH, CHIS, COAH, HGO, JAL, MÉX, MICH, NL, OAX, PUE, QRO, SLP, TAM, VER). 
*Muhlenbergia stricta (J. Presl) Kunth (COL, DGO, GTO, JAL, MÉX, MICH, MOR, NAY, OAX, TAM, VER, ZAC).

*Muhlenbergia strictior Scribn. ex Beal (CHIH, CDM, DGO, MÉX, SON).

**Muhlenbergia subaristata Swallen (DGO).

**Muhlenbergia subbiflora Hitchc. (DGO).

*Muhlenbergia tarahumara P.M. Peterson \& Columbus (CHIS, DGO).

Muhlenbergia tenella (Kunth) Trin. (CHIH, CHIS, COL, DGO, GRO, JAL, MÉX, MICH, MOR, NAY, OAX, PUE, SLP, SIN, SON, VER).

Muhlenbergia tenuifolia (Kunth) Kunth (AGS, CHIH, CHIS, COAH, CDM, DGO, GTO, GRO, HGO, JAL, MÉX, MICH, MOR, NAY, NL, OAX, PUE, QRO, SLP, SON, TAM, TLX, VER, ZAC).

Muhlenbergia tenuissima (J. Presl) Kunth (CHIS, COL, JAL, NAY, SON).

Muhlenbergia texana Buckley (BC, BCS, CHIH, DGO, NAY, SIN, SON, ZAC).

Muhlenbergia torreyi (Kunth) Hitchc. ex Bush (CHIH, COAH, DGO, NL, SLP, SON).

Muhlenbergia tricholepis (Torr.) Columbus (AGS, BC, CHIH, COAH, CDM, DGO, GTO, HGO, JAL, MÉX, MOR, NL, PUE, SLP, SON, TAM, TLX).

Muhlenbergia uniseta (Lag.) Columbus (AGS, BC, BCS, CHIH, CHIS, CDM, DGO, GTO, GRO, HGO, JAL, MICH, MOR, NAY, NL, OAX, QRO, SLP, SIN, SON, TAM, TLX, VER, ZAC).

Muhlenbergia utilis (Torr.) Hitchc. (CHIH, CHIS, COAH, CDM, DGO, GTO, HGO, JAL, MÉX, MICH, NL, PUE, QRO, SLP, SON, VER, ZAC).

Muhlenbergia vaginata Swallen $(\mathrm{CHIH}, \mathrm{CHIS}, \mathrm{CDM}, \mathrm{DGO}, \mathrm{HGO}, J A L, M E ́ X, M I C H, M O R, \mathrm{OAX}, \mathrm{PUE}, \mathrm{QRO}$, SIN, TLX, VER).

Muhlenbergia versicolor Swallen (CHIS, CDM, GTO, GRO, JAL, MÉX, MICH, MOR, NAY, OAX, PUE, VER). Muhlenbergia villiflora Hitchc.

Muhlenbergia villiflora Hitchc. var. villiflora (CHIH, COAH, DGO, HGO, NL, SLP, TAM, ZAC).

Muhlenbergia virescens (Kunth) Trin. (AGS, CHIH, COAH, DGO, GRO, GTO, HGO, JAL, MÉX, MICH, NAY, NL, QRO, SLP, SON, TAM, ZAC).

*Muhlenbergia virletii (E. Fourn.) Soderstr. (CDM, DGO, MÉX, MICH, MOR, PUE, SLP).

*Muhlenbergia watsoniana Hitchc. (AGS, DGO, JAL, SLP).

Muhlenbergia wrightii Vasey ex J.M. Coult. (BC, CHIH, COAH, DGO, SON, TAM).

${ }^{* *}$ Muhlenbergia xanthodas Soderstr. (CHIS).

Munroa (1/0/0/0; Chloridoideae)

Munroa squarrosa (Nutt.) Torr. (CHIH, COAH).

Nassella $(8 / 0 / 0 / 1 ;$ Pooideae)

Nassella cernua (Stebbins \& Löve) Barkworth (BC).

Nassella lepida (Hitchc.) Barkworth (BC, BCS, PUE).

Nassella leucotricha (Trin. \& Rupr.) R.W. Pohl (BC, CHIS, COAH, DGO, GTO, MÉX, NL, OAX, PUE, SLP, TAM, ZAC).

Nassella linearifolia (E. Fourn.) R.W. Pohl (CHIS, CDM, HGO, MÉX, OAX, PUE, TAM, TLX, VER).

Nassella mexicana (Hitchc.) R.W. Pohl (CHIS, HGO, MÉX, OAX, PUE).

Nassella mucronata (Kunth) R.W. Pohl (AGS, BC, CHIH, CHIS, COAH, CDM, DGO, GTO, HGO, JAL, MÉX, MICH, MOR, NL, OAX, PUE, QRO, SLP, TAM, TLX, VER, ZAC).

Nassella pulchra (Hitchc.) Barkworth (BCS).

+Nassella tenuissima (Trin.) Barkworth (AGS, CHIH, COAH, DGO, GTO, HGO, MÉX, NL, OAX, PUE, SLP, TAM, TLX, VER, ZAC).

Neyraudia (1/0/0/1; Chloridoideae)

+Neyraudia reynaudiana (Kunth) Keng ex Hitchc. (VER).

Ocellochloa (4/1/0/0; Panicoideae)

Ocellochloa biglandularis (Scribn. \& J. G. Sm.) Zuloaga \& Morrone (CHIS, GRO, OAX).

*Ocellochloa craterifera (Sohns) Zuloaga \& Morrone (GRO, OAX).

Ocellochloa pulchella (Raddi) Zuloaga \& Morrone (CHIS, OAX, VER).

Ocellochloa stolonifera (Poir.) Zuloaga \& Morrone (CHIS, TAB, VER).

Olmeca (5/2/1/0; Bambusoideae)

Olmeca clarkiae (Davidse \& R.W. Phol) Ruíz-Sánchez, Sosa \& Mejia-Saulés (CHIS).

*Olmeca fulgor (Soderstr.) Ruíz-Sánchez, Sosa \& Mejia-Saulés (OAX, VER).

*Olmeca recta Soderstr. (CHIS, VER).

Olmeca reflexa Soderstr. (CHIS, OAX, VER).

**Olmeca zapotecorum Ruíz-Sánchez, Sosa \& Mejia-Saulés (OAX).

Olyra (2/0/0/0; Bambusoideae)

Olyra glaberrima Raddi (CAM, CHIS, OAX, QROO, SLP, TAB, YUC). 
Olyra latifolia L. (CAM, CHIS, COL, GRO, JAL, MICH, NAY, OAX, PUE, QRO, QROO, SLP, TAB, TAM, VER, YUC).

Oplismenus (3/0/0/0; Panicoideae)

Oplismenus burmannii (Retz.) P. Beauv.

Oplismenus burmannii (Retz.) P. Beauv. var. burmannii (CHIH, CHIS, DGO, ZAC).

Oplismenus burmannii var. nudicaulis (Vasey) Mc Vaugh (BCS, CAM, CHIH, CHIS, COL, DGO, GTO,

GRO, JAL, MÉX, MICH, MOR, NAY, OAX, PUE, SIN, SON, TAB, VER, YUC, ZAC).

Oplismenus compositus (L.) P. Beauv. (CHIS, COL, DGO, GRO, JAL, MÉX, MICH, NAY, OAX, SIN, VER).

Oplismenus hirtellus (L.) P. Beauv.

Oplismenus hirtellus (L.) P. Beauv. subsp. hirtellus (AGS, BC, CAM, CHIS, JAL, MICH, MOR, PUE, QROO, SON, TAM, VER, YUC).

Oplismenus hirtellus subsp. setarius (Lam.) Mez ex E. Ekmann (CAM, CHIS, GTO, HGO, JAL, MICH, NAY, NL, OAX, PUE, QROO, SLP, VER).

Orcuttia (2/0/1/0; Chloridoideae)

Orcuttia californica Vasey (AGS, BC).

**Orcuttia fragilis Swallen (BCS).

Orthoclada (1/0/0/0; Panicoideae)

Orthoclada laxa (Rich.) P. Beauv. (CHIS, OAX, TAB, VER).

Oryza (3/0/0/2; Oryzoideae)

Oryza latifolia Desv. (CAM, CHIS, COL, GRO, JAL, NAY, OAX, SIN, TAB, VER, YUC).

+Oryza rufipogon Griff. (CHIS, NAY, OAX, VER).

+Oryza sativa L. (CAM, NAY, TAB, VER).

Otatea (11/4/6/0; Bambusoideae)

*Otatea acuminata (Munro) C.E. Calderón \& Soderstr. (CHIH, CHIS, COL, DGO, GTO, GRO, JAL, MÉX, MICH, NAY, OAX, PUE, QRO, SIN, SON, VER, ZAC).

**Otatea carrilloi Ruíz-Sánchez, Sosa \& Mejia-Saulés (CHIS).

Otatea fimbriata Soderstr. (CHIS).

** Otatea glauca L.G. Clark \& Cortés (CHIS).

**Otatea nayeeri Ruíz-Sánchez (NAY).

**Otatea ramirezii Ruíz-Sánchez (QRO).

*Otatea reynosoanna Ruíz-Sánchez \& L.G. Clark (GRO, JAL, NAY).

**Otatea rzedowskiorum Ruíz-Sánchez (CHIS).

*Otatea transvolcanica Ruíz-Sánchez \& L.G. Clark (COL, JAL, MÉX).

**Otatea victoriae Ruíz-Sánchez (HGO).

*Otatea ximenae Ruíz-Sánchez \& L.G. Clark (MICH, OAX).

Panicum (47/4/2/3; Panicoideae)

Panicum alatum Zuloaga \& Morrone

Panicum alatum Zuloaga \& Morrone var. alatum (BCS, DGO, PUE, VER).

Panicum alatum var. longiflorum Zuloaga \& Morrone (BC, SON).

Panicum alatum var. minus (Andersson) Zuloaga \& Morrone (BC, BCS, CAM, CHIH, CHIS, COAH, COL, GRO, JAL, MICH, MOR, OAX, SLP, SIN, SON, TAM, VER).

Panicum altum Hitchc. \& Chase (BCS, CAM, QROO, YUC).

Panicum amarum Elliott

Panicum amarum var. amarulum (Hitchc. \& Chase) P. Palmer (CAM, CHIS, QROO, TAB, TAM, YUC).

Panicum amarum Elliott var. amarum (QROO, TAB, TAM, VER).

+ Panicum antidotale Retz. (BC, CHIH, COAH, JAL, NL).

Panicum aquaticum Poir. (COL, QROO, VER, YUC).

*Panicum aztecanum Zuloaga \& Morrone (CDM, DGO, GRO, JAL, MÉX, MICH, SIN).

Panicum bartlettii Swallen (CAM, CHIS, HGO, OAX, QROO, SLP, TAB, VER, YUC).

Panicum capillare L. (NL, SLP, TAM).

Panicum capillarioides Vasey (NL, SLP, TAM).

Panicum cayennense Lam. (CAM, CHIS, OAX, QROO, TAB, VER).

+ Panicum coloratum L. (AGS, COAH, GRO, NL, SLP, SON).

Panicum cordovense E. Fourn. (CHIS, HGO, PUE, VER).

*Panicum decolorans Kunth (CHIS, DGO, GTO, HGO, JAL, MÉX, MICH, OAX, PUE, QRO, SLP, SIN).

Panicum dichotomiflorum Michx. (CHIH, CHIS, COL, JAL, MÉX, NAY, PUE, SIN, SON).

Panicum elephantipes Nees ex Trin. (CDM, GTO, HGO, JAL, MÉX, MICH, SLP, SIN, TAB).

Panicum ghiesbreghtii E. Fourn. (CAM, CHIS, MÉX, MICH, MOR, NAY, OAX, PUE, QROO, SLP, SIN, TAB,

TAM, VER, YUC). 
Panicum gouinii E. Fourn. (VER, YUC).

Panicum grande Hitchc. \& Chase (CHIS, TAB, VER).

Panicum haenkeanum J. Presl (CHIS, VER).

Panicum hallii Vasey

Panicum hallii var. filipes (Scribn.) F.R. Waller (COAH, HGO, MÉX, NL, OAX, PUE, QRO, SLP, TAM,

VER).

Panicum hallii Vasey var. hallii (OAX, SLP).

Panicum havardii Vasey $(\mathrm{CHIH})$.

Panicum hirsutum Sw. (CAM, CHIS, COL, OAX, QROO, SLP, TAB, TAM, VER, YUC).

Panicum hirticaule J. Presl

Panicum hirticaule J. Presl var. hirticaule (BC, CHIS, GRO, JAL, NAY, OAX, SON, TAB, VER).

Panicum hirticaule var. verrucosum Zuloaga \& Morrone $(\mathrm{CHIH}, \mathrm{COAH}, \mathrm{COL}, \mathrm{DGO}, \mathrm{GRO}, \mathrm{JAL}, \mathrm{NAY}$, SIN, SON, VER, ZAC).

Panicum hirtum Lam. (CHIS, OAX).

Panicum hispidifolium Swallen (CHIS, OAX).

Panicum hylaeicum Mez (CAM, CHIS, QROO, SLP, TAB, VER, YUC).

Panicum lepidulum Hitchc. \& Chase (AGS, CHIS, CHIH, COAH, CDM, DGO, GTO, HGO, JAL, MÉX, MICH, MOR, OAX, PUE, QRO, SLP, SON, ZAC).

**Panicum longum Hitchc. \& Chase (VER).

Panicum millegrana Poir. / Chis., Pue., (VER).

Panicum pampinosum Hitchc. \& Chase (BCS, CHIH, DGO, GTO, OAX, PUE, SON).

Panicum parcum Hitchc. \& Chase (CHIH, CHIS, COAH, COL, DGO, GTO, GRO, HGO, JAL, MÉX, MICH, MOR, NAY, OAX, PUE, SLP, SIN, TLX).

Panicum pilosum Sw. (CAM, CHIS, GRO, HGO, JAL, MOR, NAY, OAX, PUE, QRO, QROO, SLP, TAB, VER, YUC).

Panicum plenum Hitchc. \& Chase (CHIH, COAH, DGO, GRO, JAL, MOR, SON, VER).

Panicum polygonatum Schrad. (CHIS, NAY, OAX, TAB, VER).

+Panicum repens L. (CAM, TAM).

Panicum rudgei Roem. \& Schult. (TAB, VER).

Panicum sellowii Nees (CHIS, OAX, PUE, VER).

Panicum stagnatile Hitchc. \& Chase (CHIS, PUE, TAB, VER).

Panicum stramineum Hitchc. \& Chase (CHIH, CHIS, DGO, GRO, JAL, MICH, NAY, NL, OAX, SIN, SON).

Panicum sublaeve Swallen (CHIS, COL).

*Panicum tamaulipense F.R. Waller \& Morden (SLP, TAM, VER).

Panicum trichanthum Nees (CAM, CHIS, COL, OAX, PUE, QROO, SLP, TAB, TAM, VER, YUC).

Panicum trichoides Sw. (BC, BCS, CAM, CHIH, CHIS, COL, DGO, GRO, JAL, MÉX, MICH, MOR, NAY, OAX, PUE, QRO, SLP, SIN, SON, TAB, TAM, VER, YUC).

Panicum urvilleanum Kunth (BC, SON).

*Panicum vaseyanum Scribn. \& Beal (BC, BCS, CAM, CHIH, CHIS, COL, DGO, GRO, JAL, MÉX, MICH, MOR, NAY, OAX, PUE, QRO, SLP, SIN, SON, TAB, TAM, VER, YUC).

Panicum virgatum L. (BC, CHIH, CHIS, COAH, JAL, MÉX, MICH, MOR, OAX, PUE, QRO, QROO, SLP, SON, TAB, TAM, VER).

**Panicum yecorae Sánchez-Ken (SON).

Pappophorum (4/0/0/0; Chloridoideae)

Pappophorum bicolor E. Fourn. (CHIH, COAH, DGO, GTO, MÉX, NL, SLP, TAM, VER).

Pappophorum pappiferum (Lam.) Kuntze (COAH, OAX, VER).

Pappophorum philippianum Parodi (SON).

Pappophorum vaginatum Buckley (CHIH, COAH, DGO, NL, PUE, SLP, SON TAM, ZAC).

Parapholis (1/0/0/1; Pooideae)

+Parapholis incurva (L.) C.E. Hubb. (BC).

Parodiophyllochloa (1/0/0/0; Panicoideae)

Parodiophyllochloa cordovensis (E. Fourn.) Zuloaga \& Morrone (HGO, PUE, VER).

Pascopyrum (1/0/0/0; Pooideae)

Pascopyrum smithii (Rydb.) Barkworth \& D.R. Dewey (COAH).

Paspalidium (2/0/0/0; Panicoideae)

Paspalidium chapmanii (Vasey) R.W. Pohl (CAM, QROO, YUC).

Paspalidium geminatum (Forssk.) Stapf (BC, BCS, CAM, CHIS, GTO, GRO, JAL, MÉX, MOR, NAY, OAX, PUE, QROO, SIN, SON, TAB, TAM, VER, YUC). 
Paspalum (87/12/3/1; Panicoideae)

Paspalum acuminatum Raddi (CHIS, MICH, MOR, TAB, VER).

Paspalum acutum Chase (VER).

Paspalum adoperiens (E. Fourn.) Chase (CHIS, DGO, OAX, SIN, VER).

Paspalum affine Steud. (CHIS, HGO, MÉX, MOR, OAX, VER).

Paspalum alcalinum Mez (MÉX, MICH, MOR, SLP, VER, YUC).

*Paspalum arsenei Chase (AGS, CDM, HGO, JAL, MÉX, MICH, MOR, NAY, PUE, VER, ZAC).

Paspalum blodgettii Chapm. (CAM, CHIS, OAX, QROO, TAM, VER, YUC).

Paspalum botterii (E. Fourn.) Chase (CAM, CHIH, CHIS, COL, GRO, HGO, JAL, MÉX, MICH, MOR, NAY, NL, OAX, PUE, QRO, QROO, S.LP., SIN, SON, TAB, TAM, VER, YUC).

Paspalum caespitosum Flüggé (CAM, CHIS, OAX, PUE, QRO, QROO, TAB, TAM, VER, YUC).

Paspalum campylostachyum (Hack.) S. Denham (CHIS, OAX, PUE, TAB).

Paspalum candidum (Humb. \& Bonpl. ex Flüggé) Kunth (CHIS, HGO, JAL, MICH, MOR, PUE, VER).

Paspalum centrale Chase (CHIS, VER).

**Paspalum chiapense Sánchez-Ken (CHIS).

Paspalum clavuliferum C. Wright (CAM, CHIS, COL, GRO, JAL, MICH, NAY, OAX, SIN, TAM, VER, YUC).

Paspalum conjugatum P.J. Bergius (AGS, CAM, CHIS, COL, DGO, GRO, HGO, JAL, MÉX, MICH, MOR, NAY, NL, OAX, PUE, QRO, QROO, SLP, SIN, TAB, TAM, VER, ZAC).

Paspalum conspersum Schrad. (CAM, CHIS, COL, CDM, JAL, MÉX, MICH, MOR, NAY, OAX, PUE, QROO, SLP, TAM, VER).

Paspalum convexum Humb. \& Bonpl. ex Flüggé (AGS, CHIH, CHIS, COL, CDM, DGO, GTO, GRO, JAL, MÉX, MICH, MOR, NAY, OAX, PUE, QRO, SIN, SON, TAB, VER, ZAC).

Paspalum corcovadense Raddi (CAM, OAX, VER, YUC).

Paspalum coryphaeum Trin. (CAM, YUC).

Paspalum costaricense Mez (CHIS, OAX).

*Paspalum crinitum Chase (COAH, DGO, GTO, JAL, MÉX, NL, PUE, SLP ZAC).

Paspalum cymbiforme E. Fourn. (CHIS, JAL, MÉX, OAX, VER).

Paspalum decumbens Sw. (CHIS, DGO, VER).

Paspalum denticulatum Trin. (CHIS, DGO, HGO, JAL, MICH, MOR, NL, OAX, SLP, SIN, TAM, VER, ZAC).

Paspalum dilatatum Poir. (BC, CHIS, COAH, DGO, JAL, MÉX, MICH, OAX, QRO, SLP, TAM, VER).

Paspalum distichum L. (AGS, BC, CHIH, CHIS, COAH, COL, CDM, DGO, GTO, HGO, JAL, MÉX, MICH,

MOR, NAY, NL, OAX, PUE, QRO, QROO, SLP, TAB, TAM, VER, ZAC).

* Paspalum erectum Chase (COL, GTO, JAL, MICH).

Paspalum erianthum Nees ex Trin. (CHIS, GRO, OAX).

Paspalum fasciculatum Willd. ex Flüggé (CAM, CHIS, JAL, OAX, QROO, TAB, VER).

Paspalum fimbriatum Kunth (BC, CAM, MÉX, QROO, YUC).

Paspalum foliiforme S. Denham (CHIS).

*Paspalum guayanerum Beetle (NAY, SIN, SON).

Paspalum hartwegianum E. Fourn. (AGS, CHIS, GTO, GRO, JAL, MÉX, MICH, MOR, NL, OAX, PUE, QRO,

SLP, SIN, SON, TAM, VER, YUC).

Paspalum heterotrichon Trin. (CHIS).

*Paspalum hintonii Chase (COL, GRO, JAL, MÉX, MICH, NAY, OAX).

Paspalum humboldtianum Flüggé (AGS, CHIS, COAH, COL, DGO, GTO, GRO, HGO, JAL, MÉX, MICH,

MOR, NAY, OAX, PUE, QRO, SLP, SIN, SON, TAM, VER, ZAC).

Paspalum intermedium Munro ex Morong et Britton (CHIS, JAL, MÉX, MICH, MOR, NAY, VER).

Paspalum jaliscanum Chase (CHIS, COL, CDM, DGO, HGO, JAL, MICH, OAX, PUE, SLP, VER, ZAC).

Paspalum lentiginosum J. Presl (BC, BCS, CHIS, COL, DGO, HGO, JAL, MÉX, MICH, MOR, NAY, OAX, PUE, QROO, SIN, SON, VER).

*Paspalum leptachne Chase (NAY, SIN, VER).

Paspalum ligulare Nees (JAL, OAX, SIN, VER).

Paspalum lineare Trin. (CHIS, PUE, TAB).

*Paspalum longicuspe Nash (CHIS, GRO, JAL, MÉX, MICH, MOR, NAY, OAX, SIN, VER).

**Paspalum longum Chase (COL).

*Paspalum luxurians R. Guzmán \& L. Rico (GTO, JAL, MÉX).

Paspalum malacophyllum Trin. (CAM, CHIS, GTO, QRO, QROO, SLP, TAB, TAM, VER, YUC).

*Paspalum mayanum Chase (CAM, CHIS, TAB, YUC).

Paspalum melanospermum Desv. ex Poir. (JAL, NAY, OAX).

Paspalum microstachyum J. PresI (NAY, TAB, VER).

Paspalum millegrana Schrad. (CAM, CHIS, PUE, QROO, TAB, TAM, VER, YUC).

Paspalum minus E. Fourn. (CHIS, GTO, MÉX, MICH, OAX, PUE, SLP, TAB, TAM, VER).

Paspalum monostachyum Vasey (VER).

Paspalum multicaule Poir. (CHIS, GRO, JAL, MÉX, NAY, OAX, TAB, VER).

*Paspalum nelsonii Chase (CHIS, COAH, GTO, HGO, JAL, NAY, NL, OAX, PUE, QRO, SLP, TAM, VER).

Paspalum notatum Alain ex Flüggé (AGS, BC, CAM, CHIS, COL, CDM, DGO, GTO, GRO, HGO, JAL, MÉX, MICH, MOR, NAY, NL, OAX, PUE, QROO, SLP, TAB, TAM, VER, YUC, ZAC). 
Paspalum nutans Lam. (CHIS, QRO).

Paspalum orbiculatum Poir. (CHIS, GRO, OAX, SLP, SIN, TAB, VER).

Paspalum paniculatum L. (AGS, BC, BCS, CAM, CHIS, COAH, COL, JAL, MÉX, MICH, MOR, NAY, OAX, PUE, QROO, SLP, SIN, TAB, TAM, VER, YUC, ZAC).

*Paspalum paucispicatum Vasey (BC, CHIH, CDM, JAL, MÉX, NL, OAX, SON, TAB).

Paspalum pectinatum Nees ex Trin. (CHIS, OAX, TAB, VER).

Paspalum pilosum Lam. (CHIS, MÉX, MICH, OAX, TAB, VER).

Paspalum plenum Chase (CHIS, COL, JAL, MÉX, MOR, NAY, NAY, VER).

Paspalum plicatulum Michx. (AGS, CAM, CHIS, COAH, COL, DGO, GTO, GRO, HGO, JAL, MÉX, MICH, MOR, NAY, NL, OAX, PUE, QROO, SLP, SIN, TAB, TAM, VER, YUC, ZAC).

Paspalum prostratum Scribn. et Merr. (CHIS, CDM, DGO, GTO, GRO, HGO, JAL, MÉX, MICH, MOR, OAX, PUE, VER).

Paspalum pubiflorum Rupr. ex E. Fourn. (AGS, BC, BCS, CAM, CHIH, COAH, DGO, GRO, GTO, HGO, JAL, MÉX, MICH, MOR, NL, OAX, PUE, QRO, SLP, SON, TAM, VER, ZAC).

Paspalum pulchellum Kunth (CHIS, OAX, TAB, VER).

Paspalum pygmaeum Hack. (CDM, DGO, GRO, JAL, MICH, MOR, TLX, VER).

Paspalum repens P.J. Bergius (CAM, CHIS, OAX, TAB, VER).

+ Paspalum scrobiculatum L. (JAL).

Paspalum serpentinum Hochst. ex Steud. (TAB).

Paspalum setaceum Michx.

Paspalum setaceum var. ciliatifolium (Michx.) Vasey (COAH, MÉX, NL, SLP, TAB, TAM, VER).

Paspalum setaceum var. psammophyllum (Nash ex Hitchc.) D.J. Banks (VER).

Paspalum setaceum Michx. var. setaceum (TAM, VER).

Paspalum setaceum var. stramineum (Nash) D.J. Banks (CHIH, DGO, JAL, MÉX, NL, SON, TAM, VER). * Paspalum sparsum Chase (CAM, YUC).

Paspalum squamulatum E. Fourn. (BCS, CHIS, COL, GRO, JAL, MÉX, MICH, MOR, NAY, OAX, PUE, QRO, SLP, SIN, SON, TAM, VER).

Paspalum stellatum Humb. \& Bonpl. ex Flüggé (CHIS, OAX).

Paspalum tenellum Willd. (AGS, CHIS, CDM, GRO, GTO, JAL, MÉX, MICH, MOR, PUE, QRO, SON, TAM, VER, ZAC).

Paspalum tinctum Chase (CDM, GRO, GTO, JAL, MÉX, MICH, MOR, NAY, OAX, PUE, QRO).

**Paspalum tolucense R. Guzmán (MÉX).

Paspalum trachycoleon Steud. (CHIS, OAX).

Paspalum tumidum Kuhlm. (CHIS, COL, GRO, JAL, MÉX, MICH).

Paspalum turriforme R.W. Pohl (CHIS, MICH).

Paspalum unispicatum (Scribn. \& Merr.) Nash (CHIS, GRO, JAL, MOR, NL, OAX, PUE, SLP, TAM, VER).

Paspalum urvillei Steud. (AGS, BC, BC, SUR, CHIS, COAH, JAL, MÉX, MICH, MOR, NL).

Paspalum vaginatum Sw. (BCS, CAM, CHIS, COL, GRO, JAL, MÉX, MICH, NAY, OAX, PUE, QRO, QROO,

SLP, TAB, TAM, VER, YUC, ZAC).

Paspalum variabile (E. Fourn.) Nash (CHIS, COL, HGO, JAL, MÉX, MICH, MOR, OAX, PUE, QRO, TAM, VER).

Paspalum virgatum L. (CAM, CHIS, COL, GRO, JAL, MÉX, MICH, MOR, NAY, OAX, PUE, QROO, SLP, TAB, TAM, VER, YUC).

Paspalum virletii E. Fourn. (HGO, NL, PUE, QRO, SLP, SON, TAM, VER).

Paspalum wrightii Hitchc. \& Chase (CAM, TAB, YUC).

Pennisetum (2/0/0/1; Panicoideae)

Pennisetum nervosum (Nees) Trin. (CHIS, PUE, SLP, TAM, VER).

+Pennisetum villosum R. Br. ex Fresen. (AGS, CHIS, COAH, CDM, DGO, JAL, MÉX, MICH, MOR, NL, QRO, SIN, TAM, ZAC).

Peyritschia (4/0/2/0; Pooideae)

Peyritschia deyeuxioides (Kunth) Finot (AGS, CHIH, CHIS, CDM, DGO, GTO, GRO, HGO, JAL, MÉX, MICH, MOR, OAX, PUE, QRO, SLP, VER, ZAC).

**Peyritschia humilis (Louis-Marie) Finot (MÉX).

Peyritschia koelerioides (Peyr.) E. Fourn. (MÉX, MICH, OAX, PUE, VER).

Peyritschia pringlei (Scribn.) S.D. Koch (AGS, CHIH, CHIS, DGO, GTO, HGO, JAL, MÉX, PUE, QRO, SLP, SON, TLX).

Phalaris (7/0/0/3; Pooideae)

Phalaris angusta Nees ex Trin. (BC, CHIH).

+ Phalaris aquatica $\mathrm{L}$. $(\mathrm{CHIH})$.

Phalaris arundinacea L. (BC, CHIH).

+Phalaris canariensis L. (CAM, CHIH, CHIS, COAH, CDM, DGO, GTO, GRO, HGO, JAL, MÉX, MICH, NL,

OAX, PUE, SIN, TAM, TLX, VER, ZAC). 
Phalaris caroliniana Walter (BC, $\mathrm{CHIH}, \mathrm{COAH}, \mathrm{SON})$.

+Phalaris minor Retz. (BC, CHIS, GTO, HGO, JAL, MÉX, MICH, MOR, NL, PUE, QRO, SIN, SON, VER).

Phalaris paradoxa L. (BC, CHIH, JAL).

Pharus (4/0/0/0; Pharoideae)

Pharus lappulaceus Aubl. (CAM, CHIS, SLP, TAB, VER).

Pharus latifolius L. (CHIS, MÉX, OAX, QROO, SLP, TAB, VER).

Pharus mezii Prod. (CHIS, GRO, JAL, MICH, VER).

Pharus parvifolius Nash (CHIS, TAB, VER).

Phleum (2/0/0/1; Pooideae)

Phleum alpinum L. (CHIS, COAH, MÉX, NL, PUE, VER).

+Phleum pratense L. (JAL, MÉX).

Phragmites (1/0/0/0; Arundinoideae)

Phragmites australis (Cav.) Trin. ex Steud. (AGS, BC, BCS, CAM, CHIH, CHIS, COAH, COL, DGO, GTO, GRO, HGO, JAL, MÉX, MICH, MOR, NAY, NL, OAX, PUE, QRO, QROO, SLP, SIN, SON, TAB, TAM, TLX, VER, YUC, ZAC).

Phyllostachys (3/0/0/3; Bambusoideae)

+Phyllostachys aurea Rivière \& C. Rivière (CDM, VER).

+ Phyllostachys bambusoides Siebold \& Zucc. (PUE, VER).

+Phyllostachys dulcis McClure (PUE, VER).

Piptochaetium (9/1/0/0; Pooideae)

*Piptochaetium angustifolium (Hitchc.) Valencia \& Costas (COAH, MÉX, NL, TAM).

Piptochaetium avenaceum (L.) Parodi (TAM).

Piptochaetium brevicalyx (E. Fourn.) Ricker

*Piptochaetium brevicalyx (E. Fourn.) Ricker subsp. brevicalyx (GTO, HGO, MOR, NL, PUE, SLP).

*Piptochaetium brevicalyx subsp. flexuosum Barkworth (DGO).

Piptochaetium fimbriatum (Kunth) Hitchc. (AGS, BC, BCS, CAM, CHIH, CHIS, COAH, COL, CDM, DGO, GTO, HGO, JAL, MÉX, MICH, MOR, NAY, NL, OAX, PUE, QROO, SLP, SIN, SON, TAM, TLX, VER, ZAC). Piptochaetium pringlei (Beal) Parodi (BC, CHIH, COAH, DGO, GTO, NL, OAX, PUE, SLP, SON).

Piptochaetium seleri (Pilg.) Henrard (CHIS, COAH, CDM, HGO, MÉX, MICH, MOR, OAX, PUE, QRO, TAM, TLX, VER).

Piptochaetium stipoides (Trin. \& Rupr.) Hack. ex Arechav.

Piptochaetium stipoides (Trin. \& Rupr.) Hack. ex Arechav. var. stipoides (GTO, JAL, NL, PUE, SLP).

Piptochaetium uruguense Griseb. (S.L.P).

Piptochaetium virescens (Kunth) Parodi

*Piptochaetium virescens var. arsenii (Hack.) Beetle (MICH, MOR).

*Piptochaetium virescens (Kunth) Parodi var. virescens (DGO, VER, ZAC).

Poa $(23 / 1 / 3 / 3 ;$ Pooideae $)$

Poa alpina L.

Poa alpina L. subsp. alpina (OAX, PUE, VER).

+Poa annua L. (AGS, BC, BCS, CHIH, CHIS, COAH, COL, CDM, DGO, GRO, GTO, HGO, JAL, MÉX, MICH, MOR, NL, OAX, PUE, QRO, SLP, SON, TAM, TLX, VER, ZAC).

**Poa bajaensis Soreng (BC).

Poa bigelovii Vasey \& Scribn. (BC, BCS, CHIH, COAH, NL, SON).

Poa calycina (J. Presl) Kunth

Poa calycina var. mathewsii (Ball) Refulio (MÉX, PUE, SLP, TLX, VER).

Poa chamaeclinos Pilg. (MÉX, PUE).

+Poa compressa L. (COAH).

Poa fendleriana (Steud.) Vasey

Poa fendleriana subsp. albescens (Hitchc.) Soreng. (CHIH, SON).

Poa fendleriana (Steud.) Vasey subsp. fendleriana (BC, CHIH, COAH, SON).

Poa fendleriana subsp. Iongiligula (Scribn. \& T.A. Williams) Soreng (BC).

Poa gymnantha Pilg. (MÉX).

+Poa infirma Kunth (BC).

Poa matri-occidentalis P.M. Peterson \& Soreng

**Poa matri-occidentalis P.M. Peterson \& Soreng subsp. matri-occidentalis (DGO).

**Poa matri-occidentalis subsp. mohinorensis Soreng \& P.M. Peterson (CHIH).

**Poa mulleri Swallen, J. Wash. (NL).

Poa occidentalis (Vasey) Vasey (COAH).

Poa orizabensis Hitchc. (MÉX, PUE, VER). 
*Poa palmeri Soreng \& P.M. Peterson (COAH, NL).

Poa pratensis $\mathrm{L}$.

Poa pratensis subsp. agassizensis (B. Boivin \& D. Löve) Roy L. Taylor \& MacBryde (BC, COAH).

Poa pratensis subsp. alpigena (Lindm.) Hiitonen (NL).

+Poa pratensis subsp. angustifolia (L.) Lej. (SLP).

Poa pratensis subsp. irrigata (Lindm.) H. Lindb. (VER).

+Poa pratensis L. subsp. pratensis (BC, CHIH, COAH, CDM, HGO, MÉX, NL, VER).

Poa ruprechtii Peyr. (CDM, HGO, MÉX, OAX, SLP, VER).

Poa scaberula Hook. f. (CHIS, CDM, MÉX, MOR, PUE, TLX, VER).

Poa secunda J. Presl

Poa secunda subsp. juncifolia (Scribn.) Soreng. (BC).

Poa secunda J. Presl subsp. secunda (BC).

Poa seleri Pilg. (CHIS, HGO, MÉX, MOR, , OAX, PUE, VER).

Poa strictiramea Hitchc. (CHIH, COAH, DGO, ZAC).

Poa thomasii Refulio (BC).

**Poa wendtii Soreng \& P.M. Peterson (COAH).

Polypogon (6/0/0/3; Pooideae)

Polypogon australis Brongn. (BC).

Polypogon elongatus Kunth (AGS, CHIH, CHIS, COAH, COL, CDM, DGO, GTO, JAL, MÉX, MICH, MOR, NL, OAX, PUE, QRO, SLP, TAM, VER, ZAC).

Polypogon interruptus Kunth (BC, BCS, CDM, DGO, GTO, HGO, JAL, MÉX, MICH, NL, OAX, PUE, TLX). +Polypogon maritimus Willd. (BC, NL, SON).

+Polypogon monspeliensis (L.) Desf. (AGS, BC, CHIH, CHIS, COAH, CDM, DGO, GTO, HGO, JAL, MÉX, $\mathrm{MICH}, \mathrm{OAX}, \mathrm{PUE}, \mathrm{QRO}, \mathrm{TAM}, \mathrm{ZAC}$ ).

+Polypogon viridis (Gouan) Breistr. (AGS, BC, BCS, CHIH, CHIS, COAH, CDM, DGO, GTO, GRO, HGO, JAL, MÉX, MICH, MOR, NL, OAX, PUE, QRO, SLP, SON, TAM, VER, ZAC).

Pseudechinolaena (1/0/0/0; Panicoideae)

Pseudechinolaena polystachya (Kunth) Stapf (CHIS, GRO, HGO, OAX, PUE, TAB, VER).

Reimarochloa (1/0/0/0; Panicoideae)

Reimarochloa oligostachya (Munro ex Benth.) Hitchc. (COL, TAB).

Rhipidocladum (4/0/1/0; Bambusoideae)

Rhipidocladum bartlettii (McClure) McClure (CAM, CHIS, QROO).

**Rhipidocladum martinezii Davidse \& R.W. Pohl (CHIS).

Rhipidocladum pittieri (Hack.) McClure (CHIS).

Rhipidocladum racemiflorum (Steud.) McClure (DGO, JAL, MICH, NAY, OAX, SIN, TAM, VER).

Rhytachne (2/0/0/0; Panicoideae)

Rhytachne guianensis (Hitchc.) Clayton (CHIS, OAX).

Rhytachne rottboellioides Desv. ex Ham. (CHIS, TAB).

Rostraria (1/0/0/1; Pooideae)

+ Rostraria cristata (L.) Tzvelev (BC).

Rottboellia (1/0/0/1; Panicoideae)

+ Rottboellia cochinchinensis (Lour.) Clayton (CAM, OAX, QROO, TAB, VER).

Saccharum (2/0/0/1; Panicoideae)

+Saccharum officinarum L. (BCS, CAM, CHIS, COL, GRO, JAL, MOR, NAY, OAX, PUE, QROO, TAB, TAM, VER, YUC, ZAC).

Saccharum villosum Steud. (CHIS).

Sacciolepis (2/0/0/0; Panicoideae)

Sacciolepis myuros (Lam.) Chase (CHIS, JAL, MÉX, NAY, OAX, VER).

Sacciolepis striata (L.) Nash (TAB, VER).

Schedonorus (1/0/0/1; Pooideae)

+Schedonorus arundinaceus (Schreb.) Dumort. (CDM, HGO, MÉX, ZAC).

Schismus (2/0/0/2; Danthonioideae)

+ Schismus arabicus Nees (BC, CHIH, SON).

+ Schismus barbatus (L.) Thell. (BC, CHIH, SON). 
Schizachyrium (13/2/1/0; Panicoideae)

Schizachyrium brevifolium (Sw.) Nees ex Büse (CHIH, CHIS, COL, JAL, MÉX, MICH, MOR, NAY, OAX, SIN, TAB, VER, YUC, ZAC).

Schizachyrium cirratum (Hack.) Wooton \& Standl. (AGS, BCS, CHIH, CHIS, COAH, CDM, DGO, GTO, GRO, HGO, JAL, MÉX, MICH, MOR, NL, OAX, PUE, QRO, SLP, SON, TAM, TLX, VER, ZAC).

Schizachyrium condensatum (Kunth) Nees (CAM, CHIS, HGO, JAL, MÉX, MICH, MOR, NAY, OAX, PUE, QROO, SLP, SIN, TAB, TAM, VER).

*Schizachyrium gaumeri Nash (CAM, CHIS, QROO, YUC).

Schizachyrium malacostachyum (J. Presl) Nash (BCS, CAM, CHIS, GRO, JAL, MOR, NAY, OAX, PUE, VER, $\mathrm{YUC}$ ).

*Schizachyrium mexicanum (Hitchc.) A. Camus (CHIH, CHIS, COL, GTO, JAL, MÉX, MICH, MOR, NAY, NL, SON, VER).

Schizachyrium microstachyum (Desv. ex Ham.) Roseng., B.R. Arrill. \& Izag. (CAM, CHIS, CDM, GRO, HGO, JAL, MÉX, MICH, MOR, NAY, OAX, PUE, QRO, QROO, SLP, SIN, SON, TAB, TAM, VER, YUC).

**SChizachyrium muelleri Nash (VER).

Schizachyrium salzmanni (Trin. ex Steud.) Nash (CHIS, OAX, TAB, TAM, VER).

Schizachyrium sanguineum (Retz.) Alston (AGS, BC, BCS, CAM, CHIH, CHIS, COAH, COL, CDM, DGO, GTO, GRO, JAL, MÉX, MICH, MOR, NAY, NL, OAX, PUE, QRO, SLP, SIN, SON, TAB, TAM, TLX, VER, YUC, ZAC).

Schizachyrium scoparium (Michx.) Nash

Schizachyrium scoparium var. littorale (Nash) Gould (CAM, PUE, TAB, TAM, VER).

Schizachyrium scoparium var. neomexicanum (Nash) Hitchc. (CHIH, COAH, DGO, NL, SLP).

Schizachyrium scoparium (Michx.) Nash var. scoparium (TAM).

Schizachyrium semitectum (Swallen) Reeder (BC, BCS, CHIH, CHIS, GRO, JAL, MÉX, MOR, OAX, SON, YUC).

Schizachyrium tenerum Nees (AGS, CHIH, CHIS, CDM, DGO, GTO, GRO, HGO, JAL, MÉX, MICH, MOR, NAY, OAX, PUE, QRO, SLP, SIN, TAM, VER, ZAC).

Scleropogon (1/0/0/0; Chloridoideae)

Scleropogon brevifolius Phil. (AGS, CHIH, COAH, DGO, GTO, HGO, NL, PUE, QRO, SLP, SON, TAM, VER, ZAC).

Secale $(1 / 0 / 0 / 1 ;$ Pooideae)

+Secale cereale L. (BC, CDM, GTO, GRO, HGO, JAL, MÉX, MICH, MOR, NAY, NL, TAM, VER).

Setaria (28/1/1/2; Panicoideae)

Setaria adhaerens (Forssk) Chiov. (AGS, BC, BCS, CHIH, COAH, CDM, DGO, GTO, JAL, MÉX, NL, OAX, PUE, QRO, ZAC).

Setaria arizonica Rominger (SON).

Setaria faberi R.A.W. Herrm. (MÉX).

Setaria grisebachii E. Fourn. (AGS, BCS, CAM, CHIH, CHIS, COAH, COL, CDM, DGO, GTO, HGO, JAL, MÉX, MICH, MOR, NAY, NL, OAX, PUE, QRO, QROO, SLP, SON, TAM, TLX, VER, YUC, ZAC).

*Setaria latifolia (Scribn.) R.A.W. Herrm. (DGO, GTO, JAL, MICH, OAX).

Setaria leucopila (Scribn. \& Merr.) K. Schum. (BC, BCS, CHIH, COAH, DGO, GTO, NL, PUE, QRO, SLP, SON, TAM, ZAC).

Setaria liebmannii E. Fourn. (BCS, CHIH, CHIS, COL, DGO, GRO, JAL, MÉX, MICH, MOR, NAY, OAX, PUE, SIN, SON, VER).

Setaria longipila E. Fourn. (CHIS, JAL, MICH, NAY, OAX).

Setaria macrosperma (Scribn. \& Merr.) K. Schum. (DGO, NL, TAM).

Setaria macrostachya Kunth (BC, BCS, CHIH, CHIS, COAH, DGO, HGO, MÉX, MICH, MOR, NAY, NL, OAX, PUE, QRO, SLP, SON, TAM, VER, ZAC).

Setaria magna Griseb. (CAM, COAH, QROO, YUC).

**Setaria palmeri Henrard (BCS).

Setaria palmifolia (J. Konig) Stapf (BCS, CHIS, JAL, MICH, NAY, OAX, PUE, QRO, QROO, QRO, SLP, SON, TAB, VER).

Setaria parviflora (Poir.) Kerguélen (BC, CAM, CHIH, CHIS, COAH, CDM, DGO, GTO, HGO, MÉX, MICH, MOR, NL, OAX, QRO, QROO, SLP, SON, TAB, TAM, VER, YUC, ZAC).

Setaria pumila (Poir.) Roem. \& Schult. (DGO).

Setaria reverchonii (Vasey) Pilg.

Setaria reverchonii subsp. firmula (Hitchc. \& Chase) W.E. Fox (COAH).

Setaria reverchonii subsp. ramiseta (Scribn.) W.E. Fox (COAH, TAM).

Setaria scandens Schrad. (CAM, CHIS, MÉX, OAX, VER, YUC).

Setaria scheelei (Steud.) Hitchc. (CHIH, CHIS, COAH, DGO, HGO, JAL, MICH, NL, PUE, SLP, TAM, VER).

Setaria setosa (Sw.) P. Beauv. (MICH, NL). 
+ Setaria sphacelata (Schumach.) Stapf \& C.E. Hubb. ex M.B. Moss

+ Setaria sphacelata var. sericea (R.E. Massey ex Stapf) Clayton (TAB).

+Setaria sphacelata (Schumach.) Stapf \& C.E. Hubb. ex M.B. Moss. var. sphacelata (MÉX).

Setaria sulcata Raddi (OAX, VER).

Setaria tenax (Rich.) Desv. (CAM, CHIS, GRO, JAL, NL, OAX, PUE, QROO, SLP, TAB, VER, YUC).

Setaria texana Emery (NL, TAM).

Setaria variifolia (Swallen) Davidse (CAM, QROO, YUC).

Setaria verticillata (L.) P. Beauv. (BCS, CHIH, COAH, DGO, OAX, PUE, VER).

Setaria villosissima (Scribn. \& Merr) K. Schum. (CHIH, COAH, NL, SON, TAM).

+Setaria viridis (L.) P. Beauv. (BC, CHIH, DGO, MOR, PUE, SLP, SON, TAM, VER).

Setaria vulpiseta (Lam.) Roem. \& Schult. (CAM, CHIS, OAX, PUE, QROO, SLP, SON, VER, YUC).

Setariopsis (2/1/0/0; Panicoideae)

Setariopsis auriculata (E. Fourn.) Scribn. (BC, BCS, CAM, CHIH, CHIS, COL, GTO, GRO, JAL, MÉX, MICH, MOR, NAY, OAX, PUE, QROO, SLP, SIN, SON, VER, YUC).

*Setariopsis latiglumis (Vasey) Scribn. (CHIH, CHIS, GTO, GRO, JAL, MÉX, MICH, MOR, NAY, OAX, PUE, ZAC).

Sohnsia (1/1/0/0; Chloridoideae)

*Sohnsia filifolia (E. Fourn.) Airy Shaw (QRO, SLP).

Sorghastrum (7/0/2/0; Panicoideae)

Sorghastrum brunneum Swallen (CHIS, COAH, DGO, GRO, HGO, MICH, NAY, NL, OAX, PUE, QRO, SLP, TAM, VER).

Sorghastrum incompletum (J. Presl) Nash

Sorghastrum incompletum (J. Presl) Nash var. incompletum (CAM, CHIS, COL, HGO, JAL, MICH, MOR,

NAY, OAX, YUC, ZAC).

**Sorghastrum nudipes Nash $(\mathrm{CHIH})$.

Sorghastrum nutans (L.) Nash (CHIH, CHIS, COAH, GTO, GRO, JAL, MÉX, MICH, NAY, OAX, PUE, TAM,

VER, ZAC).

**Sorghastrum pohlianum Dávila, L. I. Cabrera \& Lira (COL).

Sorghastrum setosum (Griseb.) Hitchc. (CAM, CHIS, QRO, TAB, VER, YUC).

Sorghastrum stipoides (Kunth) Nash (CHIS, OAX).

Sorghum (3/0/0/2; Panicoideae)

+Sorghum bicolor (L.) Moench (BC, CAM, CHIH, CHIS, COL, DGO, GRO, JAL, MÉX, MICH, MOR, NAY, NL, OAX, PUE, QROO, TAM, VER, YUC, ZAC).

+Sorghum halepense (L.) Pers. (CAM, CHIH, CHIS, COAH, DGO, GRO, HGO, MÉX, MOR, NAY, NL, OAX, QRO, QROO, SLP, SIN, SON, TAM, VER, YUC, ZAC).

Sorghum trichocladum (Rupr. ex Hack.) Kuntze (CHIS, COL, GRO, JAL, MICH, NAY, OAX, SIN).

Sphenopholis (2/0/0/0; Pooideae)

Sphenopholis interrupta (Buckley) Scribn.

**Sphenopholis interrupta subsp. californica (Vasey) Scribn. (BC).

Sphenopholis interrupta (Buckley) Scribn. subsp. interrupta (BC).

Sphenopholis obtusata (Michx.) Scribn. (COAH, CDM, DGO, GTO, NL, OAX, PUE, SON).

Sporobolus (34/5/3/2; Chloridoideae)

Sporobolus airoides (Torr.) Torr.

Sporobolus airoides (Torr.) Torr. var. airoides (BC, CHIH, COAH, DGO, NL, SLP, ZAC).

**Sporobolus airoides subsp. regis (I.M. Johnst.) Wipff \& S.D. Jones (COAH).

*Sporobolus atrovirens (Kunth) Kunth (AGS, BC, BCS, COAH, CDM, DGO, GTO, HGO, JAL, MÉX, OAX,

PUE, QRO, QROO, SLP, TAM, TLX, VER, YUC, ZAC).

Sporobolus buckleyi Vasey (CAM, NL, PUE, QROO, TAB, TAM, VER, YUC).

Sporobolus coahuilensis Valdés-Reyna (COAH, DGO).

Sporobolus compositus (Poir.) Merr. (COAH).

Sporobolus contractus Hitchc. (BC, BCS, CHIH, COAH, DGO, JAL, NL, SLP, SON).

Sporobolus cryptandrus (Torr.) A. Gray (BC, BCS, CHIH, COAH, DGO, NL, SON, TAM, ZAC).

Sporobolus cubensis Hitchc. (CHIS, OAX, TAB, VER).

Sporobolus cynosuroides (L.) P.M. Peterson \& Saarela (CHIH, TAM).

Sporobolus domingensis (Trin.) Kunth (QROO, YUC).

Sporobolus flexuosus (Thurb. ex Vasey) Rydb. (BC, CHIH, COAH, SIN, SON).

Sporobolus foliosus (Trin.) P.M. Peterson \& Saarela (BC, BCS).

Sporobolus giganteus Nash (CHIH, COAH). 
**Sporobolus hintonii Hartley (MÉX).

Sporobolus hookerianus P.M. Peterson \& Saarela (CHIH, JAL, MICH).

Sporobolus indicus (L.) R. Br.

Sporobolus indicus (L.) R. Br. var. indicus (AGS, CHIH, CHIS, COAH, COL, CDM, DGO, GTO, GRO,

HGO, JAL, MÉX, MICH, MOR, NAY, NL, OAX, PUE, QRO, QROO, SLP, SIN, SON, TAM, TLX, VER,

YUC, ZAC).

Sporobolus junceus (P. Beauv.) Kunth (CHIS, VER).

Sporobolus macrospermus Scribn. ex Beal (AGS, CHIS, DGO, GTO, GRO, JAL, MÉX, MICH, OAX, ZAC).

Sporobolus nealleyi Vasey (COAH, NL, SLP).

+Sporobolus niliacus (Fig. \& De Not.) P.M. Peterson (BC).

*Sporobolus palmeri Scribn. (COAH, DGO, SLP, ZAC).

**Sporobolus potosiensis Wipff \& S.D. Jones. (SLP).

Sporobolus pumilus (Roth) P.M. Peterson \& Saarela (CAM, QROO, TAB, TAM, VER).

Sporobolus purpurascens (Sw.) Ham. (CHIS, COL, OAX, PUE, VER).

Sporobolus pyramidatus (Lam.) Hitchc. (AGS, BC, BCS, CHIH, CHIS, COAH, CDM, DGO, GTO, MÉX, MICH, OAX, PUE, QRO, QROO, SON, TAM, VER, YUC, ZAC).

+ Sporobolus schoenoides (L.) P.M. Peterson (BC).

Sporobolus spartinus (Trin.) P.M. Peterson \& Saarela (CAM, COAH, NL, OAX, QROO, SLP, TAB, TAM, VER, YUC).

*Sporobolus spiciformis Swallen (CHIH, COAH, CDM, DGO).

*Sporobolus splendens Swallen (CHIS, COL, JAL, NAY, OAX).

Sporobolus tenuissimus (Mart. ex Schrank) Kuntze (CHIS, MICH).

*Sporobolus trichodes Hitchc. (AGS, CHIH, DGO, GTO, GRO, JAL, MÉX, MICH, MOR, VER, ZAC).

Sporobolus virginicus (L.) Kunth (BC, BCS, CAM, CHIS, JAL, NL, OAX, QROO, SON, TAB, TAM, VER, YUC). **Sporobolus viscidus Sohns / MÉX.

Sporobolus wrightii Munro ex Scribn. (AGS, BC, BCS, CHIH, COAH, DGO, HGO, NL, PUE, SLP, SON, TAM, VER, ZAC).

Stapfochloa (2/0/0/1; Chloridoideae)

+ Stapfochloa canterae (Arechav.) P.M. Peterson (CHIS).

Stapfochloa ciliata (Sw.) P.M. Peterson (CAM, CHIS, COAH, HGO, NL, OAX, PUE, QRO, QROO, SLP, TAM, VER, YUC).

Steinchisma (2/1/0/0; Panicoideae)

*Steinchisma cupreum (Hitchc. \& Chase) W.V. Br. (DGO, JAL, MÉX, PUE, QRO, VER, ZAC).

Steinchisma hians (Elliott) Nash (CHIS, DGO, GTO, JAL, MICH).

Stenotaphrum (1/0/0/0; Panicoideae)

Stenotaphrum secundatum (Walter) Kuntze (AGS, BC, BCS, CAM, CHIH, CHIS, JAL, MICH, MOR, PUE, QROO, TAB, VER, YUC).

Stephostachys (1/0/0/0; Panicoideae)

Stephostachys mertensii (Roth) Zuloaga \& Morrone (JAL, TAB, VER).

Streptochaeta (2/0/0/0; Anomochlooideae)

Streptochaeta sodiroana Hack. (CHIS, TAB).

Streptochaeta spicata Schrad. ex Nees (CHIS, OAX, VER).

Streptogyna (1/0/0/0; Oryzoideae)

Streptogyna americana C.E. Hubb. (CHIS, VER).

Tetrapogon (2/0/1/0; Chloridoideae)

**Tetrapogon brandegei (Vasey) P.M. Peterson (BCS).

Tetrapogon chlorideus (J. Presl) P.M. Peterson (BC, BCS, CHIS, CDM, GTO, JAL, MÉX, MICH, MOR, NAY, NL, OAX, SLP, SIN, SON, TAM, VER, ZAC).

Thinopyrum (1/0/0/1; Pooideae)

+Thinopyrum intermedium (Host) Barkworth \& D.R. Dewey $(\mathrm{CHIH})$.

Thrasya (1/0/0/0; Panicoideae)

Thrasya campyylostachya (Hack.) Chase (CHIS, OAX, PUE, TAB, VER).

Trachypogon (1/0/0/0; Panicoideae)

Trachypogon spicatus (L. f.) Kuntze (CAM, CHIH, DGO, OAX, VER, YUC, ZAC). 
Tragus (1/0/0/1; Chloridoideae)

+Tragus berteronianus Schult. (AGS, BC, BCS, CHIH, COAH, CDM, DGO, GTO, HGO, JAL, MÉX, NL, OAX, PUE, QRO, SLP, SON, TAM, ZAC).

Trichanthecium (1/0/0/0; Panicoideae)

Trichanthecium cyanescens (Nees ex Trin.) Zuloaga \& Morrone (CHIS).

Trichoneura (1/0/0/0; Chloridoideae) Trichoneura elegans Swallen (TAM).

Tridens (3/0/0/1; Chloridoideae)

Tridens albescens (Vasey) Wooton \& Standl. (CHIH, COAH, NL, TAM).

+Tridens flavus (L.) Hitchc. (NL).

Tridens texanus (S. Watson) Nash (CHIH, COAH, NL, SLP, TAM).

Tridentopsis (1/0/0/0; Chloridoideae)

Tridentopsis mutica (Torr.) P.M. Peterson (BC, CHIH, COAH, DGO, NL, QRO, SLP, SON, TAM, YUC, ZAC).

Triniochloa (5/3/1/0; Pooideae)

*Triniochloa gracilis Gómez-Sánchez \& Gonz.-Led. (GRO, OAX).

*Triniochloa laxa Hitchc. (CHIH, CHIS).

*Triniochloa micrantha (Scribn.) Hitchc. (MÉX, MOR).

Triniochloa stipoides (Kunth) Hitchc. (CHIS, CDM, GTO, GRO, HGO, JAL, MÉX, MICH, MOR, OAX, PUE, QRO, SLP, VER).

**Triniochloa talpensis Gonz.-Led. \& Gómez-Sánchez (JAL).

Triplasiella (1/0/0/0; Chloridoideae)

Triplasiella eragrostoides (Vasey \& Scribn.) P.M. Peterson \& Romasch. (COAH, NL, OAX, TAM, YUC).

Triplasis (1/0/0/0; Chloridoideae)

Triplasis purpurea (Walter) Chapm. (TAB, VER).

Tripogonella (1/0/0/0; Chloridoideae)

Tripogonella spicata (Nees) P.M. Peterson \& Romasch. (AGS, CAM, CDM, DGO, GTO, JAL, MÉX, QRO, SLP, VER, ZAC).

Tripsacum (13/1/1/0; Panicoideae)

Tripsacum andersonii J.R. Gray (CAM, CHIS, VER).

*Tripsacum bravum J.R. Gray (GRO, JAL, MÉX, NAY).

Tripsacum dactyloides (L.) L.

Tripsacum dactyloides (L.) L. var. dactyloides (AGS, CHIS, COAH, CDM, DGO, GRO, JAL, MÉX, MICH, MOR, NAY, NL, OAX, PUE, SLP, TAM).

Tripsacum dactyloides var. hispidum (Hitchc.) de Wet \& J.R. Harlan (CHIS, COL, CDM, DGO, GTO, GRO, JAL, MÉX, MICH, MOR, NL, PUE, QRO, SLP, SIN, TAM).

Tripsacum dactyloides var. mexicanum de Wet \& J.R. Harlan (CAM, JAL, MICH, NAY, QROO, YUC).

Tripsacum floridamum. Porter ex Vasey (TAM).

Tripsacum intermedium de Wet \& J.R. Harlan (CHIS, GRO).

Tripsacum jalapense de Wet \& Brink (AGS, CHIS, GRO, JAL, OAX).

Tripsacum lanceolatum Rupr. ex E. Fourn. (AGS, BC, CAM, CHIH, DGO, HGO, JAL, MÉX, MICH, OAX, PUE, SLP, SIN, SON, ZAC).

Tripsacum latifolium Hitchc. (CHIS, MICH, NAY, OAX, PUE, QROO).

Tripsacum laxum Nash (CHIS, COL, GRO, JAL, MICH, NAY, OAX, VER).

Tripsacum maizar Hern.-Xol. \& Randolph (CHIS, COL, GTO, GRO, JAL, NAY, OAX).

**Tripsacum manisuroides de Wet \& J.R. Harlan (CHIS).

Tripsacum pilosum Scribn. \& Merr. (CHIH, CHIS, COL, DGO, GRO, JAL, MÉX, MICH, NAY, OAX, SLP, ZAC). Tripsacum zopilotense Hern.-Xol. \& Randolph (CHIH, CHIS, COL, DGO, GTO, GRO, JAL, MÉX, OAX, PUE, SLP, SIN, TLX, ZAC).

Trisetum (16/6/3/0; Pooideae)

Trisetum angustum Swallen (CHIS).

**Trisetum barbatipaleum (Hultén ex Veldkamp) Finot (HGO).

**Trisetum curvisetum Morden \& Valdés-Reyna (NL).

**Trisetum durangense Finot \& P. M. Peterson (DGO). 
*Trisetum filifolium Scribn. ex Beal

*Trisetum filifolium Scribn. ex Beal var. filifolium (CHIH, DGO, HGO, MÉX).

*Trisetum filifolium var. aristatum Scribn. ex Beal (CHIH, CHIS, COAH, DGO, HGO, MÉX). Trisetum irazuense (Kuntze) Hitchc. (CHIS, CDM, DGO, HGO, MÉX, OAX, PUE, SLP, VER).

*Trisetum ligulatum Finot \& Zuloaga (JAL, VER).

*Trisetum martha-gonzaleziae P. M. Peterson \& Finot (CHIH, DGO).

*Trisetum palmeri Hitchc. (CHIH, COAH, DGO, JAL, NL, SIN, SON).

Trisetum pinetorum Swallen (CHIS).

Trisetum pringlei (Scribn. ex Beal) Hitchc. (CHIS, OAX, PUE).

Trisetum rosei Scribn. \& Merr. (CHIS, HGO, MÉX, PUE, VER).

*Trisetum spellenbergii Soreng, Finot \& P. M. Peterson (CHIH, SON).

Trisetum spicatum (L.) K. Richt. (CHIH, CHIS, COAH, CDM, DGO, HGO, JAL, MÉX, MICH, NL, PUE, QRO, SLP, VER).

Trisetum viride (Kunth) Kunth (CHIH, CHIS, COAH, DGO, GRO, GTO, JAL, MÉX, MICH, OAX, QRO).

*Trisetum virletii E. Fourn. (CHIH, CHIS, CDM, DGO, GTO, GRO, HGO, JAL, MÉX, MICH, MOR, NL, PUE, QRO, SLP, VER).

Tristachya (5/1/3/0; Panicoideae)

**Tristachya angustifolia Hitchc. (NAY).

Tristachya avenacea (J. Presl) Scribn. \& Merr. (CHIS, GRO, JAL, MÉX, MICH, NAY, OAX).

**Tristachya contrerasii R. Guzmán (JAL).

*Tristachya laxa Scribn. \& Merr. (DGO, NAY, SIN).

**Tristachya papilosa R. Guzmán (NAY).

Triticum (1/0/0/1; Pooideae)

+Triticum aestivum L. (AGS, BC, BCS, CAM, CHIH, CHIS, COAH, COL, DGO, GTO, GRO, HGO, JAL, MÉX, MICH, MOR, NAY, NL, OAX, PUE, QRO, QROO, SLP, SIN, SON, TAB, TAM, TLX, VER, YUC, ZAC).

Uniola (2/0/0/1; Chloridoideae)

+Uniola paniculata L. (CHIS, TAB, TAM, VER).

Uniola pittieri Hack. (BC, BCS, CHIS, COL, JAL, NAY, OAX, SIN, SON, VER).

Urochloa (18/5/1/3; Panicoideae)

Urochloa arizonica (Scribn. \& Merr.) Morrone \& Zuloaga (BC, BCS, CHIH, COAH, DGO, GRO, JAL, NAY, $\mathrm{NL}, \mathrm{OAX}, \mathrm{SIN}, \mathrm{SON}, \mathrm{TAM})$.

Urochloa brizantha (Hochst. ex A. Rich.) R.D. Webster (CAM, NAY, PUE, YUC).

Urochloa ciliatissima (Buckley) R.D. Webster (NL, TAM).

*Urochloa discifera (E. Fourn.) Morrone \& Zuloaga (AGS, DGO, GTO, JAL, MICH, NAY, OAX, SLP).

*Urochloa distachya (L.) T.Q. Nguyen (CHIS, JAL, NL, OAX, TAB, TAM, VER, YUC).

Urochloa fusca (Sw.) B.F. Hansen \& Wunderlin (BC, BCS, CAM, CHIH, CHIS, COAH, COL, DGO, GTO, GRO, HGO, JAL, MÉX, MICH, MOR, NAY, NL, OAX, PUE, QRO, QROO, SLP, SIN, SON, TAM, VER, YUC, ZAC).

*Urochloa jaliscana (J. Santana) Espejo \& López-Ferrari (JAL, MÉX).

+Urochloa maxima (Jacq.) R.D. Webster (CAM, NAY, PUE, YUC).

*Urochloa meziana (Hitchc.) Morrone \& Zuloaga (AGS, CHIH, COAH, COL, CDM, DGO, GTO, HGO, JAL, MÉX, MICH, NL, OAX, PUE, QRO, SLP, TAM, VER, ZAC).

Urochloa mollis (Sw.) Morrone \& Zuloaga (CAM, CHIS, COL, GRO, MÉX, NAY, OAX, PUE, SIN, VER, YUC). +Urochloa mutica (Forssk.) T.Q. Nguyen (BC, BCS, CAM, CHIS, COL, GRO, HGO, JAL, MÉX, MICH, NAY, OAX, PUE, QROO, SLP, TAB, TAM, VER, YUC).

*Urochloa ophryodes (Chase) Morrone \& Zuloaga (NL, SLP, TAM).

+Urochloa panicoides P. Beauv. (AGS, JAL, MICH).

Urochloa plantaginea (Link) R.D. Webster (AGS, CHIH, CHIS, COL, DGO, GTO, GRO, JAL, MÉX, MICH,

MOR, NAY, OAX, PUE, QRO, SLP, TAM, VER, ZAC).

Urochloa platyphylla (Munro ex C. Wright) R.D. Webster (OAX).

Urochloa reptans (L.) Stapf (CAM, CHIS, COL, GRO, JAL, MICH, MOR, NAY, NL, OAX, PUE, SLP, SIN, SON, TAM, VER, YUC).

Urochloa texana (Buckley) R.D. Webster (CHIH, COAH, DGO, NAY, NL, TAM, ZAC).

**Urochloa venosa (Swallen) Morrone \& Zuloaga (MICH).

Vulpia (4/0/0/2; Pooideae)

+Vulpia bromoides (L.) Gray (BC, BCS, CHIS, CDM, MÉX, MICH, OAX, PUE, VER).

Vulpia microstachys (Nutt.) Munro

Vulpia microstachys var. ciliata (Gray) Lonard \& Gould (BC).

Vulpia microstachys var. pauciflora (Scribn. ex Beal) Lonard \& Gould (BC, BCS). 
+Vulpia myuros (L.) C.C. Gmel., Fl. Bad. 1: 8. 1805.

+Vulpia myuros var. hirsuta Hack. (BC, CHIH, CHIS, COAH, DGO, HGO, MÉX, MICH, MOR, OAX, PUE, SLP, SON, ZAC).

+Vulpia myuros var. megalura (Nutt.) Auquier (BCS).

Vulpia myuros (L.) C.C. Gmel. var. myuros (BC).

Vulpia octoflora (Walter) Rydb.

Vulpia octoflora var. glauca (Nutt.) Fernald (BC).

Vulpia octoflora var. hirtella (Piper) Henrard (BC, BCS).

Vulpia octoflora (Walter) Rydb. var. octoflora (BC, BCS).

Zea (4/2/0/0; Panicoideae)

*Zea diploperennis H.H. Iltis, Doebley \& R. Guzmán (JAL, VER).

Zea luxurians (DGOieu \& Asch.) R.M. Bird (CHIS, OAX).

Zea mays L.

Zea mays L. subsp. mays (AGS, BC, BCS, CAM, CHIH, CHIS, COAH, COL, CDM, DGO, GTO, GRO, HGO, JAL, MEX, MICH, MOR, NAY, NL, OAX, PUE, QRO, QROO, SLP, SIN, SON, TAB, TAM, TLX, VER, YUC, ZAC).

*Zea mays subsp. mexicana (Schrad.) H. H. Iltis (CHIH, CDM, DGO, GTO, GRO, JAL, MÉX, MICH, N.L., OAX, PUE, QRO).

Zea mays subsp. parvig/umis H.H. Iltis \& Doebley (GRO, JAL, MÉX, MICH).

*Zea perennis (Hitchc.) Reeves \& Mangelsd. (JAL, MÉX, MICH).

Zeugites (9/4/2/0; Panicoideae)

Zeugites americanus Willd.

Zeugites americanus Willd. var. americanus (CAM, YUC).

Zeugites americanus var. mexicanus (Kunth) McVaugh (CHIS, COL, GRO, HGO, JAL, MICH, NAY, OAX, PUE, SLP, TAM, VER).

Zeugites americanus var. pringlei (Scribn.) McVaugh (CHIS, GRO, JAL, MÉX, MICH, MOR, OAX, PUE). *Zeugites capillaris (Hitchc.) Swallen (COL, GRO, JAL, MÉX, VER).

*Zeugites hackelii Swallen (JAL, MICH, SIN).

**Zeugites hintonii T.G. Hartley (MÉX).

*Zeugites latifolius (E. Fourn.) Hemsl. (CHIS, GRO, JAL, OAX).

Zeugites munroanus Hemsl., Biol. Cent.-Amer. (CHIS, VER).

Zeugites pittieri Hack. (CHIS).

**Zeugites sagittatus Hartley (MÉX).

*Zeugites smilacifolius Scribn. (GRO, JAL, MÉX, MICH, MOR).

Zizaniopsis (1/0/0/0; Oryzoideae)

Zizaniopsis miliacea (Michx.) Döll \& Asch. (CHIS, VER).

Zoysia (1/0/0/1; Chloridoideae)

+Zoysia matrella (L.) Merr. (NAY).

Zuloagaea (1/0/0/0; Panicoideae)

Zuloagaea bulbosa (Kunth) Bess (CHIH, COAH, DGO, GRO, JAL, MOR, SON, VER). 